\title{
Tetranuclear palladacycles of 3-acetyl-7-methoxy-2H-chromen-2-one derived Schiff bases: Efficient catalysts for Suzuki-Miyaura coupling in an aqueous medium
}

Sivadasan Dharani, ${ }^{\ddagger}$ Giriraj Kalaiarasi, ${ }^{\ddagger}$ Dharmalingam Sindhuja, ${ }^{\dagger}$ Vincent M. Lynch, ${ }^{\mp}$ Ramasamy Shankar, ${ }^{\ddagger}$ Ramasamy Karvembu, ${ }^{+*}$ and Rathinasabapathi Prabhakaran ${ }^{* *}$

Department of Chemistry, Bharathiar University, Coimbatore, 641 046, India.

Department of Chemistry, National Institute of Technology, Tiruchirappalli, 620015, India.

${ }^{\mp}$ Department of Chemistry, University of Texas, Austin, TX 78712-1224, USA.

${ }^{1}$ Department of Physics, Bharathiar University, Coimbatore, 641 046, India.

*E-mail: rpnchemist@gmail.com(R. Prabhakaran)

*E-mail: kar@ nitt.edu(R. Karvembu)

\section{SUPPORTING INFORMATION}

\section{Infrared spectroscopy}

The IR spectra of the complexes showed characteristic stretching vibrations of the respective groups. The lactone carbonyl $(\mathrm{C}=\mathrm{O})^{\mathrm{S} 1}$ of the coumarin system displayed its stretching around 1641-1644 $\mathrm{cm}^{-1}$. The vibrations corresponding to $\mathrm{C}=\mathrm{N}^{\mathrm{S} 1}$ and $\mathrm{C}-\mathrm{S}^{\mathrm{S} 2}$ groups were observed in the ranges of $1577-1593 \mathrm{~cm}^{-1}$ and $755-763 \mathrm{~cm}^{-1}$ respectively.

\section{Electronic spectroscopy}

The electronic spectra of the new palladium(II) complexes (1-4) exhibited three absorption bands in the region around 263-432 nm (Figure S1). The bands that appeared in the region 263-329 nm were assigned to intra ligand transition, ${ }^{\mathrm{S} 3}$ while the bands around $419-432 \mathrm{~nm}$ were assigned to MLCT. ${ }^{\mathrm{S} 4}$ In addition, the electronic spectra were recorded in dichloromethane at three different concentrations ( $2 \mu \mathrm{M}, 5 \mu \mathrm{M}$ and $10 \mu \mathrm{M})$ and the corresponding spectral data has been provided in the Figure S2 and Table S1. The complex 1 exhibited bands around 230 (intra ligand transition), 365 (intra ligand transition) and $425 \mathrm{~nm}$ (MLCT). The complexes 2-4 showed absorptions around 229-234 nm (intra ligand transition), 322-328 nm (intra ligand transition) and 432-440 nm (MLCT). In addition to these bands, the complex 4 showed band at $249 \mathrm{~nm}$ at concentrations of 5 and $10 \mu \mathrm{M}$, which is also due to the intra ligand transition. ${ }^{\mathrm{S} 4}$ 


\section{${ }^{1}$ H NMR spectroscopy}

${ }^{1} \mathrm{H}$ NMR spectra of the ligands and complexes were recorded in DMSO (Figures S3-S6). The spectra suggested CNS chelation of the ligand in complexes 1-3 and ONS chelation in complex 4. The hydrazinic proton of the ligands were observed as a singlet around $\delta 10.26-10.77 \mathrm{ppm}$. However, no such resonance was found in the case of complexes 1-4, indicating the coordination of thiolate sulphur to palladium ion after enolization followed by deprotonation of $\mathrm{NH}-\mathrm{C}=\mathrm{S}$ group. ${ }^{\mathrm{S5}-\mathrm{S} 7}$ The ligands contained a singlet at $\delta 2.24-2.33 \mathrm{ppm}$ owing to the presence of $\mathrm{N}=\mathrm{C}$ $\mathrm{CH}_{3}$ proton. This signal has undergone a slight downfield shift at around $\delta$ 2.57-2.71 ppm, which represent the binding of azomethine nitrogen to metal. ${ }^{\mathrm{S}-\mathrm{S} 10}$ The $\mathrm{C} 4-\mathrm{H}$ proton of the coumarin system was displayed as a singlet at $\delta 7.98-8.45 \mathrm{ppm}$ in the ligands $\mathbf{H}_{2} \mathbf{L}^{\mathbf{1 . 4}} \cdot{ }^{\mathrm{S} 6}$ Complete disappearance of this peak in the complexes 1-3 depicted the coordination of $\mathrm{C} 4$ carbon atom to the palladium ion. In complex $\mathbf{4}$, the signal for the same has been appeared at $\delta 7.01 \mathrm{ppm}$ as a singlet, showing the non-participation of $\mathrm{C} 4-\mathrm{H}$ in coordination. Besides these signals, the ligands and complexes exhibited signals for aromatic protons in the range of $\delta 6.29-8.04 \mathrm{ppm}$ and a singlet for $-\mathrm{OCH}_{3}$ at $\delta 3.74-3.97 \mathrm{ppm} .{ }^{\mathrm{S} 11}$ In addition, the spectra of complexes $\mathbf{1}$ and $\mathbf{2}$ contained a singlet at $\delta 9.00$ and $9.05 \mathrm{ppm}$ which may be due to the aldehydic proton of DMF, present in the crystal lattice. However at present, we are unable to offer any reason for the shift in the above said chemical shift value from the normally observed value at 7.95-8.02 ppm of DMF in DMSO.

\section{${ }^{1} \mathrm{H}$ NMR spectral data of complexes 1-4 in $\mathrm{CDCl}_{3}$ (with two drops of DMSO-d6)}

Complex 1: ${ }^{1} \mathrm{H}$ NMR (400 MHz, $\mathrm{CDCl}_{3}, \delta$ ppm, $J$ Hz): 8.80 (s, 1H, C5-H), 7.09 (d, 2H, C6-H \&

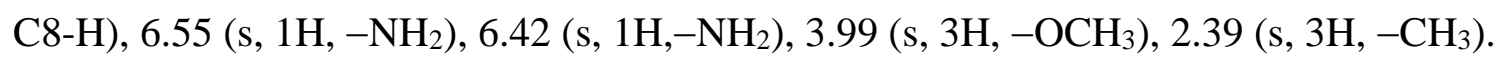

Complex 2: ${ }^{1} \mathrm{H}$ NMR (400 MHz, $\mathrm{CDCl}_{3}, \delta$ ppm, $\left.J \mathrm{~Hz}\right): 8.82(\mathrm{~s}, 1 \mathrm{H}, \mathrm{C} 5-\mathrm{H}), 7.88(\mathrm{~d}, J=7.6 \mathrm{~Hz}$, $1 \mathrm{H},-\mathrm{NH}), 7.07-7.20$ (m, 2H, C6-H \& C8-H), 3.99 (s, 3H, $-\mathrm{OCH}_{3}$ ), 2.91 (s, 3H, NH-CH3), 2.69 $\left(\mathrm{s}, 3 \mathrm{H},-\mathrm{CH}_{3}\right)$.

Complex 3: ${ }^{1} \mathrm{H}$ NMR (400 MHz, $\mathrm{CDCl}_{3}, \delta$ ppm, $J \mathrm{~Hz}$ ): 8.78 (s, $\left.1 \mathrm{H}, \mathrm{C} 5-\mathrm{H}\right), 7.86(\mathrm{~d}, J=8.8 \mathrm{~Hz}$, 1H, -NH), 7.06-7.14 (m, 2H, C6-H \& C8-H), 3.99 (s, 3H, - $\left.\mathrm{OCH}_{3}\right), 3.35$ (d, J = 5.2 Hz, 2H, $\left.\mathrm{CH}_{2}\right), 2.68\left(\mathrm{~s}, 3 \mathrm{H},-\mathrm{CH}_{3}\right), 1.18-1.21\left(\mathrm{t}, J=6.8 \mathrm{~Hz}, 3 \mathrm{H}\right.$, terminal $\left.-\mathrm{CH}_{3}\right)$. 
Complex 4: ${ }^{1} \mathrm{H}$ NMR (400 MHz, $\mathrm{CDCl}_{3}, \delta$ ppm, $J$ Hz): 9.52 (s, 1H, C4-H), 8.87 (s, 1H, C5-H), 7.89 (s, 1H, -NH), 7.28-7.32 (t, $J=7.2 \mathrm{~Hz}, 2 \mathrm{H}, \mathrm{C6}-\mathrm{H} \& \mathrm{C} 8-\mathrm{H}), 7.01-7.16$ (m, 5H, phenyl protons), $4.01\left(\mathrm{~s}, 3 \mathrm{H},-\mathrm{OCH}_{3}\right), 2.78\left(\mathrm{~s}, 3 \mathrm{H},-\mathrm{CH}_{3}\right)$.

\section{X-ray crystallography}

\section{Hydrogen bonding}

Each monomeric units of complexes $\mathbf{1}$ and $\mathbf{3}$ showed two hydrogen bondings - the hydrogen atom $\mathrm{H}(1)$ of $\mathrm{N}(3)-\mathrm{H}(1)$ is in intermolecular hydrogen bonding with ring carbonyl oxygen atom $\mathrm{O}(2)$ of the adjacent tetrameric unit. Similarly the $\mathrm{O}(2)$ atom of one molecule forms an intermolecular hydrogen bonding with $\mathrm{H}(1)-\mathrm{N}(3)$ of next molecule $[\mathrm{O}(2)-\mathrm{N}(3)=3.017 \AA$ for complex 1 and $\mathrm{O}(2)-\mathrm{N}(3)=2.971 \AA$ for complex 3] (Table S4). As a whole, one molecule contains eight intermolecular hydrogen bonds. This hydrogen bonding leads to an unremitting formation of 3D chain (Fig. S18 and S20). Similarly, in complex 2 each monomeric unit involves two intermolecular hydrogen bodings one between the ring carbonyl oxygen atom $\mathrm{O}(1)$ of one molecule and hydrogen atom of $\mathrm{N}(6) \mathrm{H}$ of the adjacent molecule, and the other is between the hydrogen atom of $\mathrm{N}(3) \mathrm{H}$ of one molecule and $\mathrm{O}(4)$ oxygen atom of next molecule $[\mathrm{O}(1)-\mathrm{N}(6)=$ $2.906 \AA$ and $\mathrm{N}(3)-\mathrm{O}(4)=2.882 \AA]$. All these hydrogen bondings gave a 3D chain formation (Figure S19).

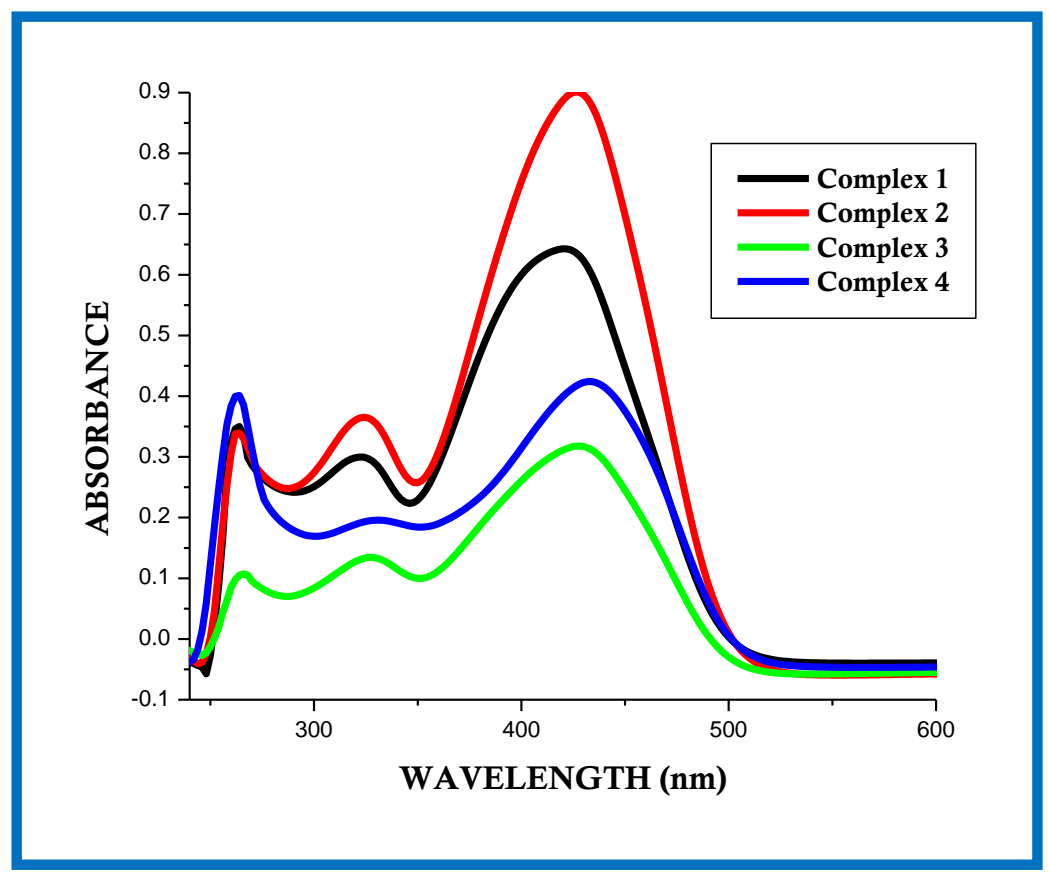

Figure S1. Electronic spectra of the complexes 1-4 


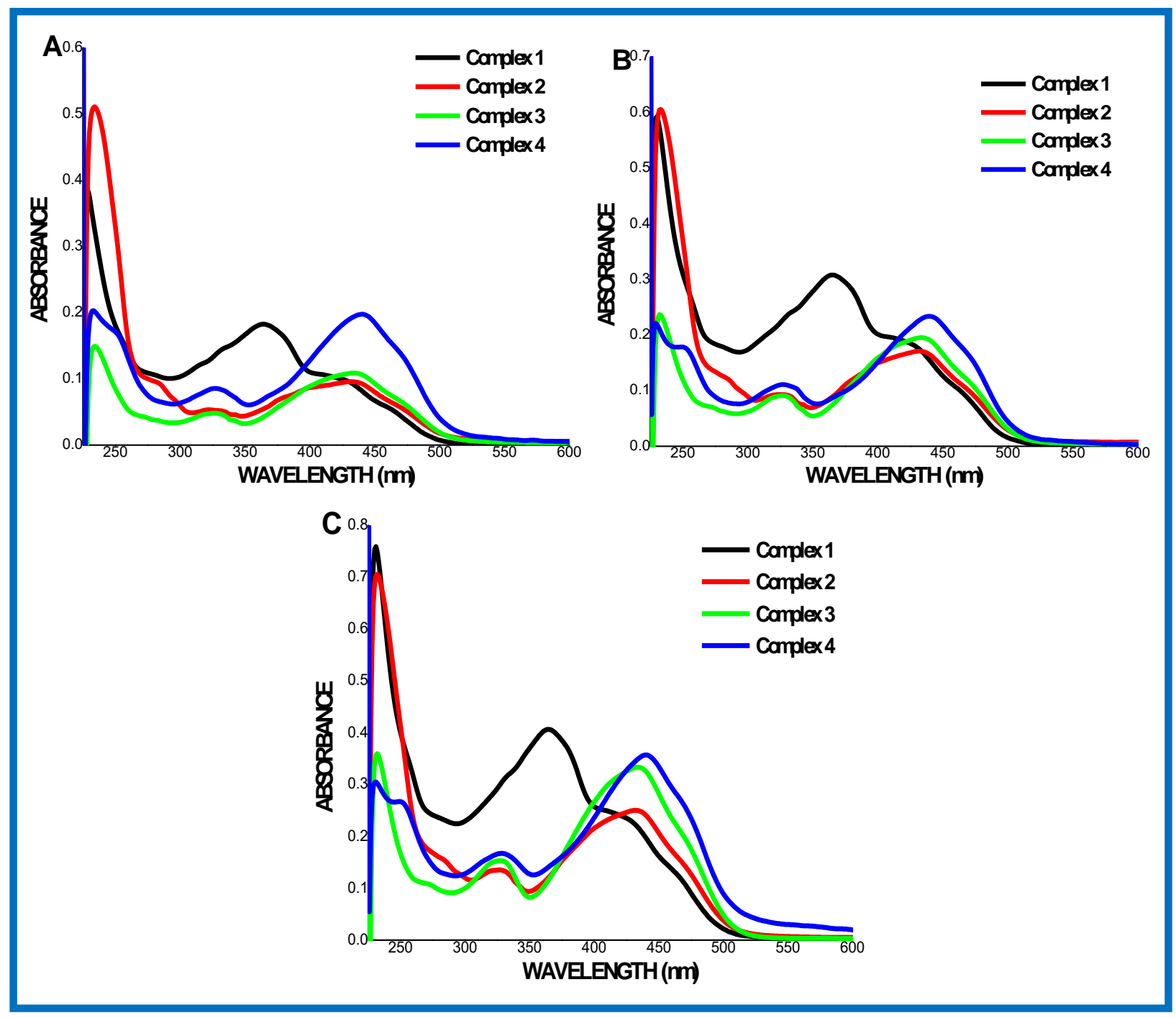

Figure S2. Electronic spectra of the complexes 1-4 recorded in dichloromethane at different concentrations: $\mathrm{A}=2 \mu \mathrm{M} ; \mathrm{B}=5 \mu \mathrm{M}$ and $\mathrm{C}=10 \mu \mathrm{M}$. 


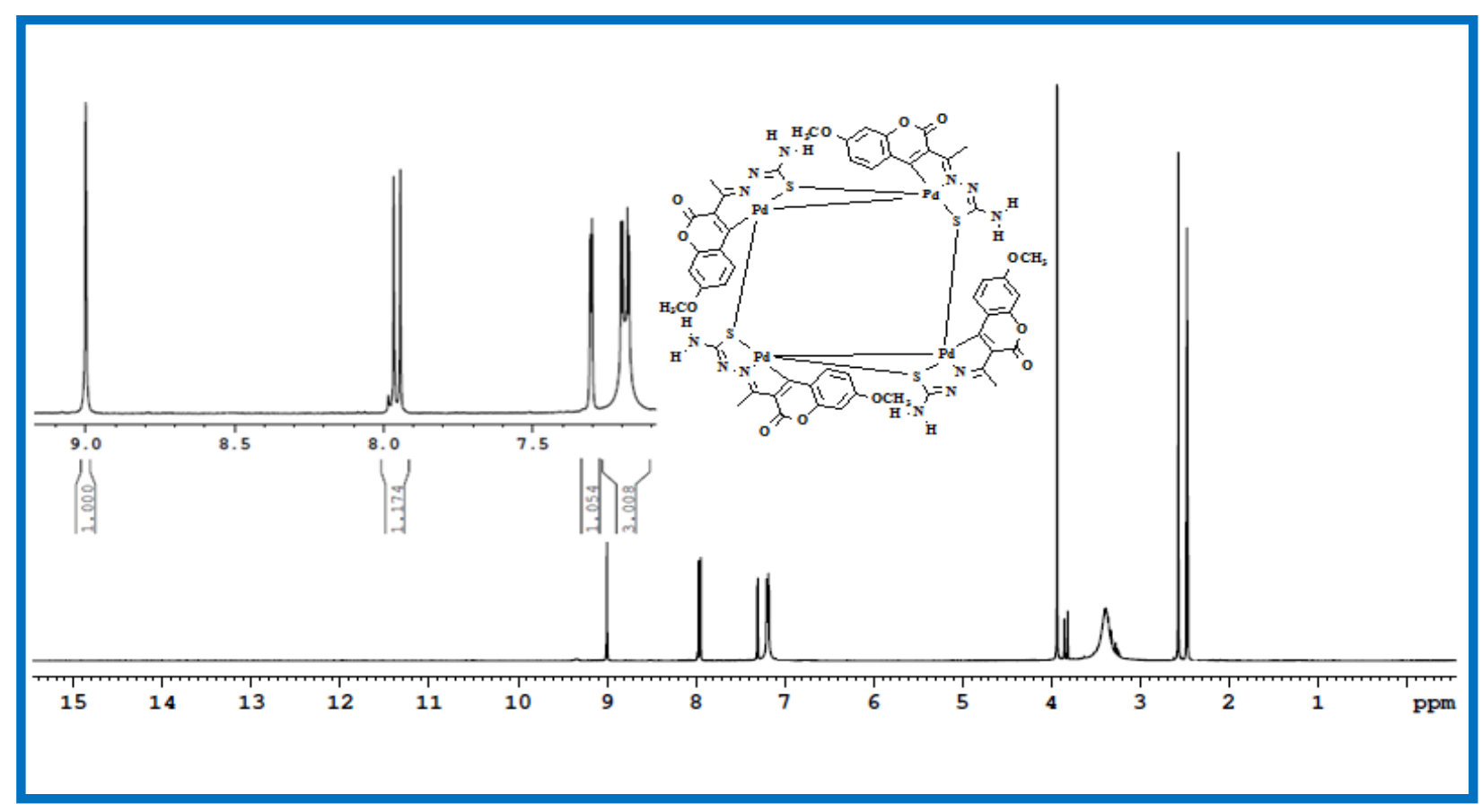

Figure S3. ${ }^{1} \mathrm{H}$ NMR spectrum of complex 1 in DMSO-d 6

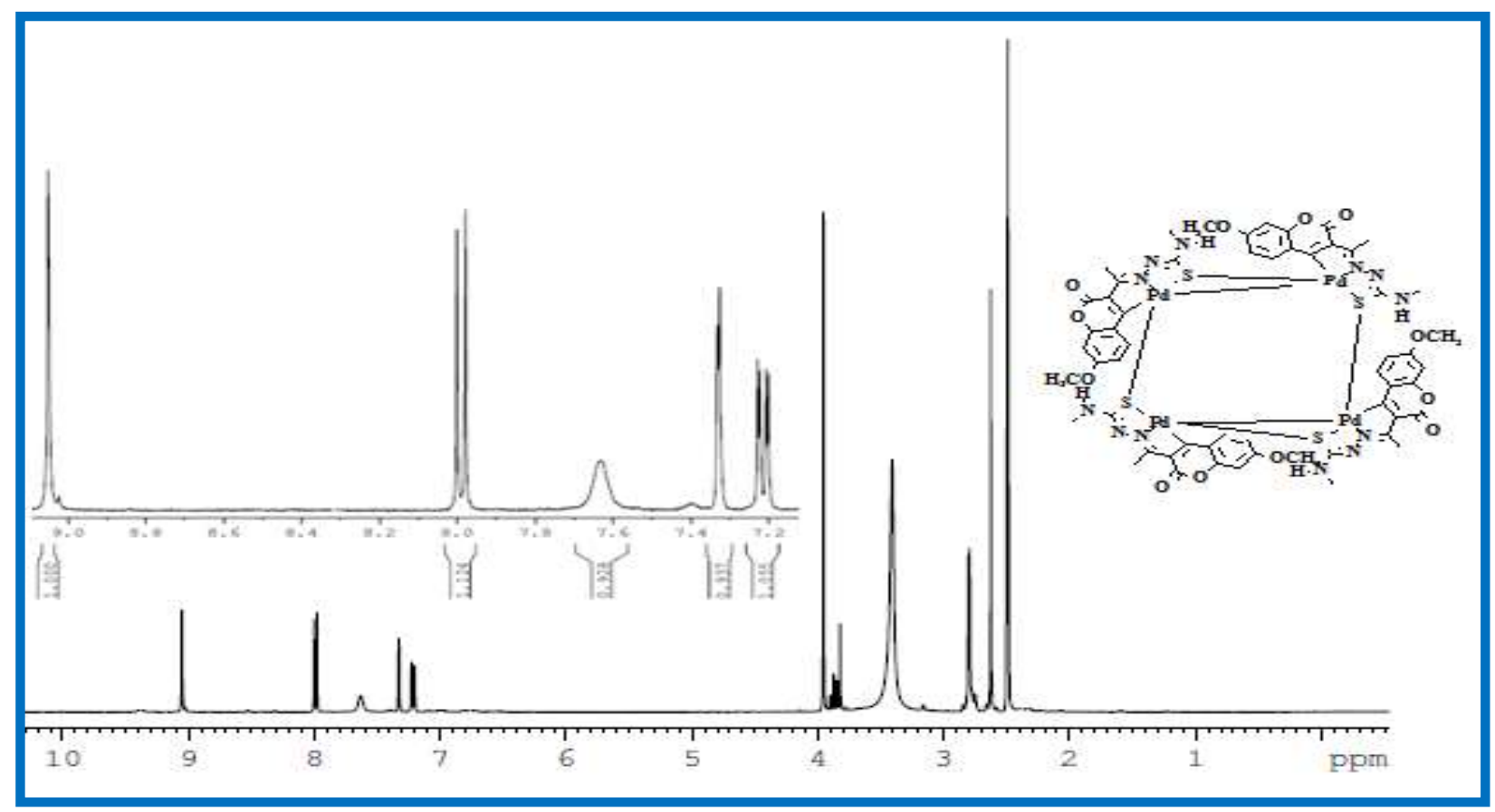

Figure S4. ${ }^{1} \mathrm{H}$ NMR spectrum of complex 2 in DMSO-d 6 


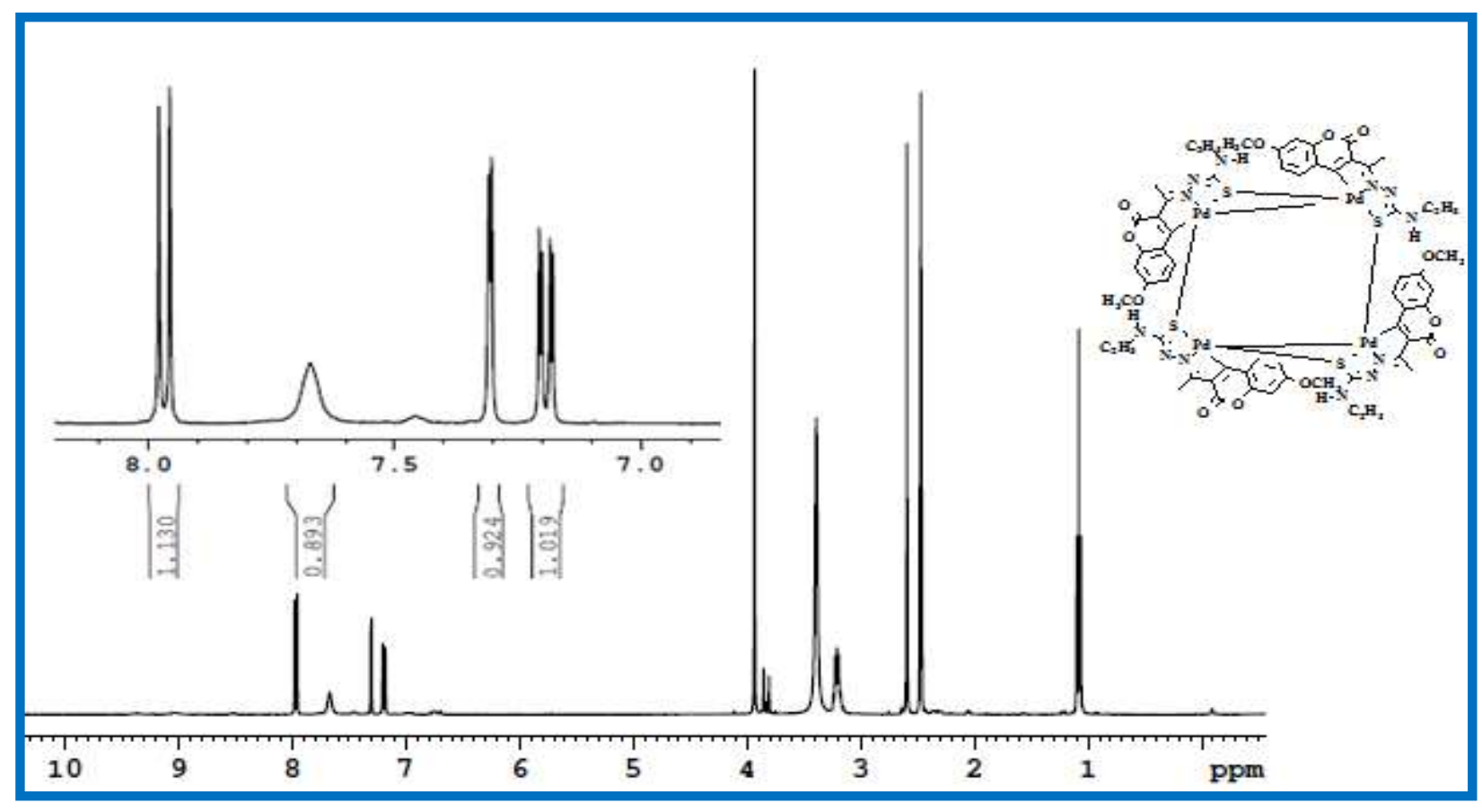

Figure S5. ${ }^{1} \mathrm{H}$ NMR spectrum of complex 3 in DMSO-d 6

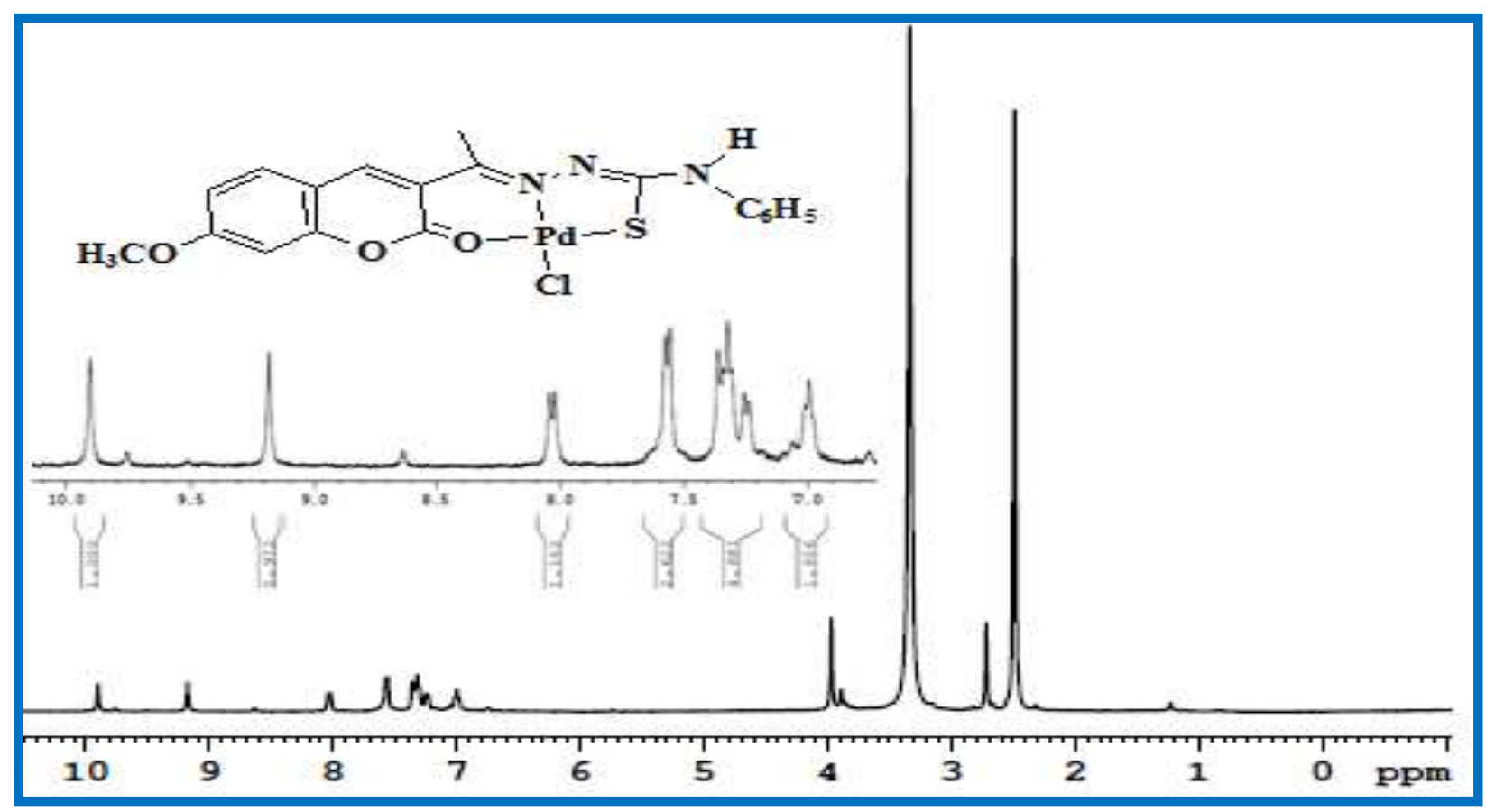

Figure S6. ${ }^{1} \mathrm{H}$ NMR spectrum of complex 4 in DMSO-d 6 


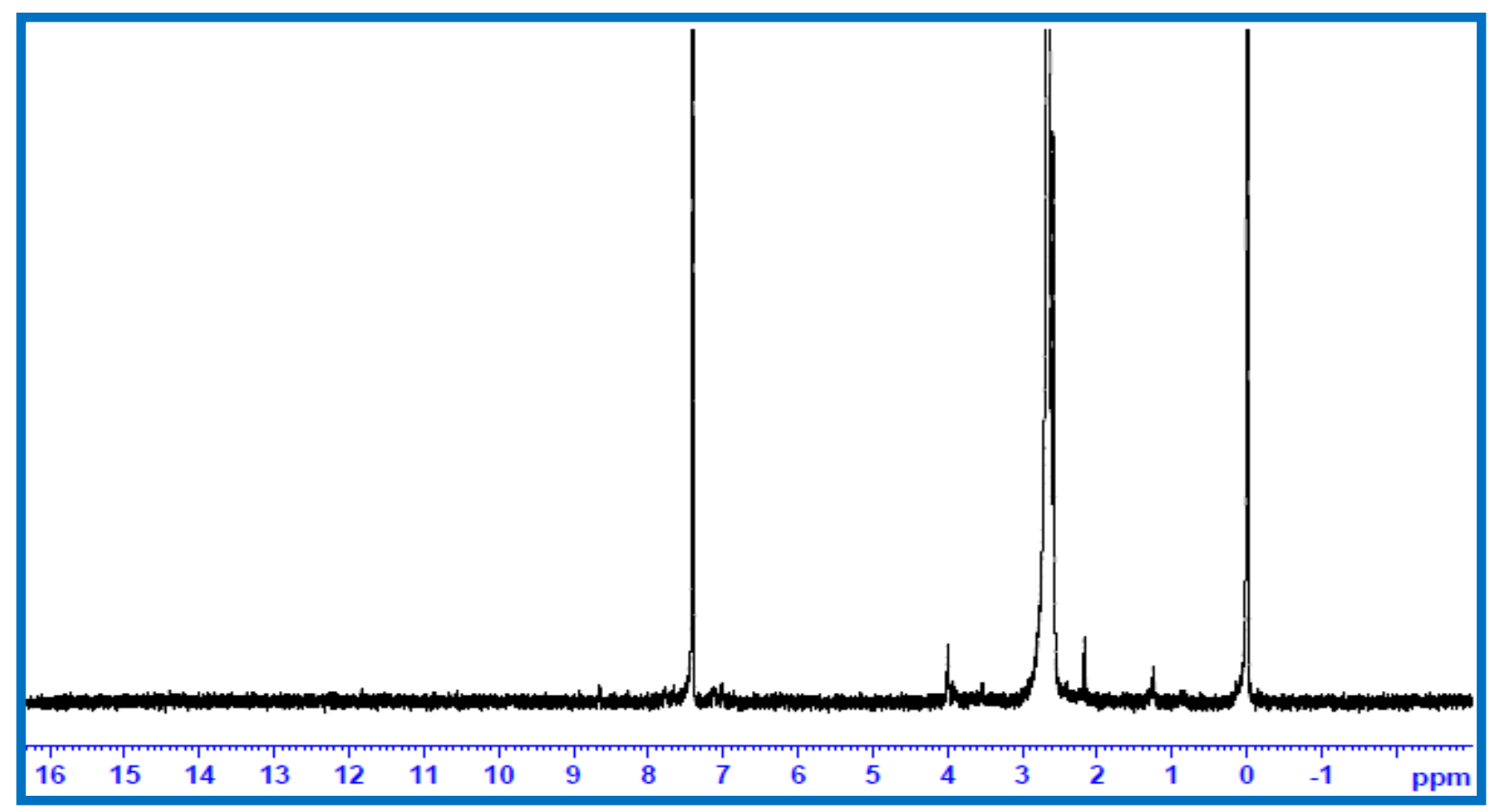

Figure S7. ${ }^{1} \mathrm{H}$ NMR spectrum of complex 1 in $\mathrm{CDCl}_{3}$

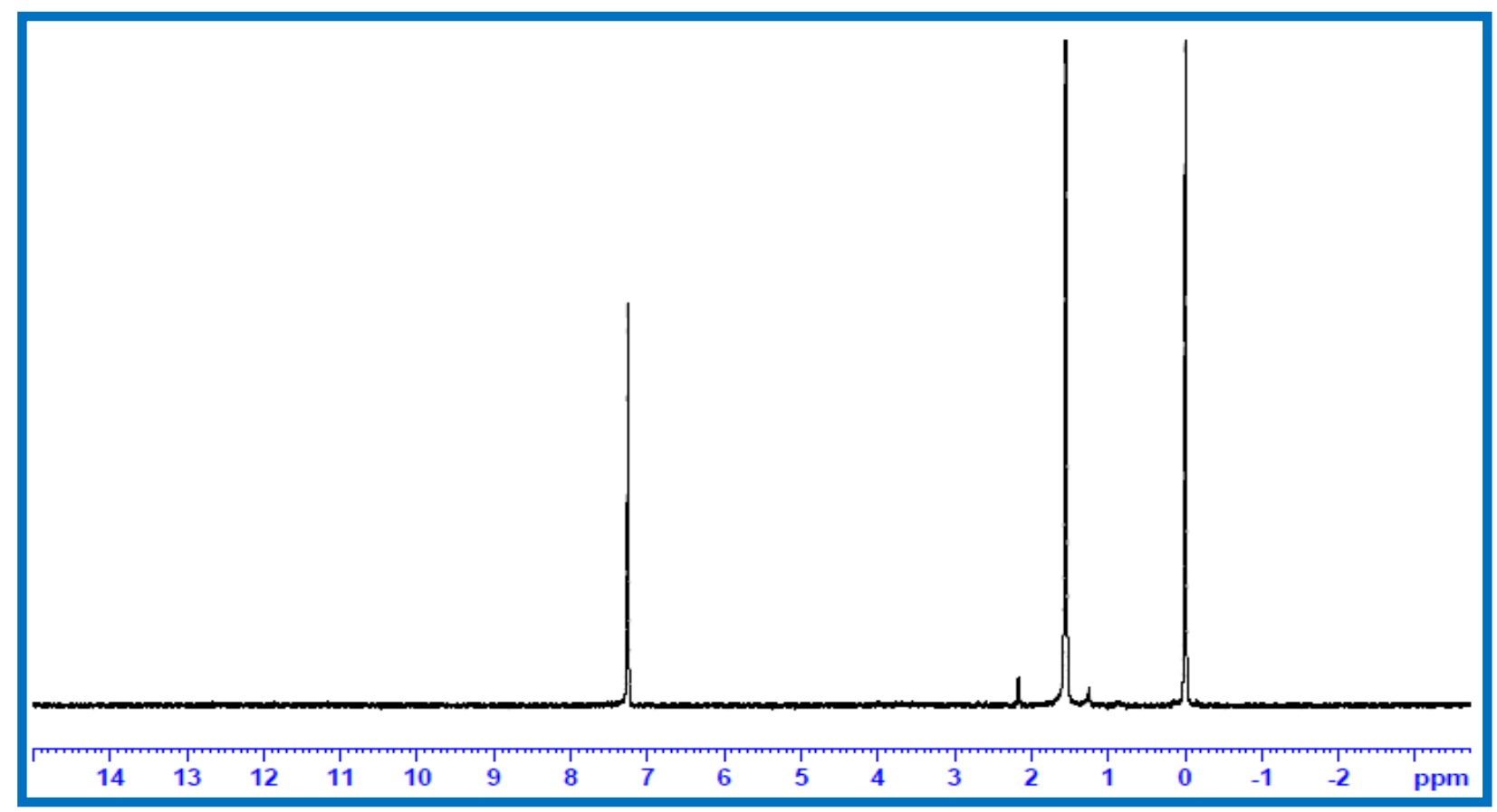

Figure S8. ${ }^{1} \mathrm{H}$ NMR spectrum of complex 2 in $\mathrm{CDCl}_{3}$ 


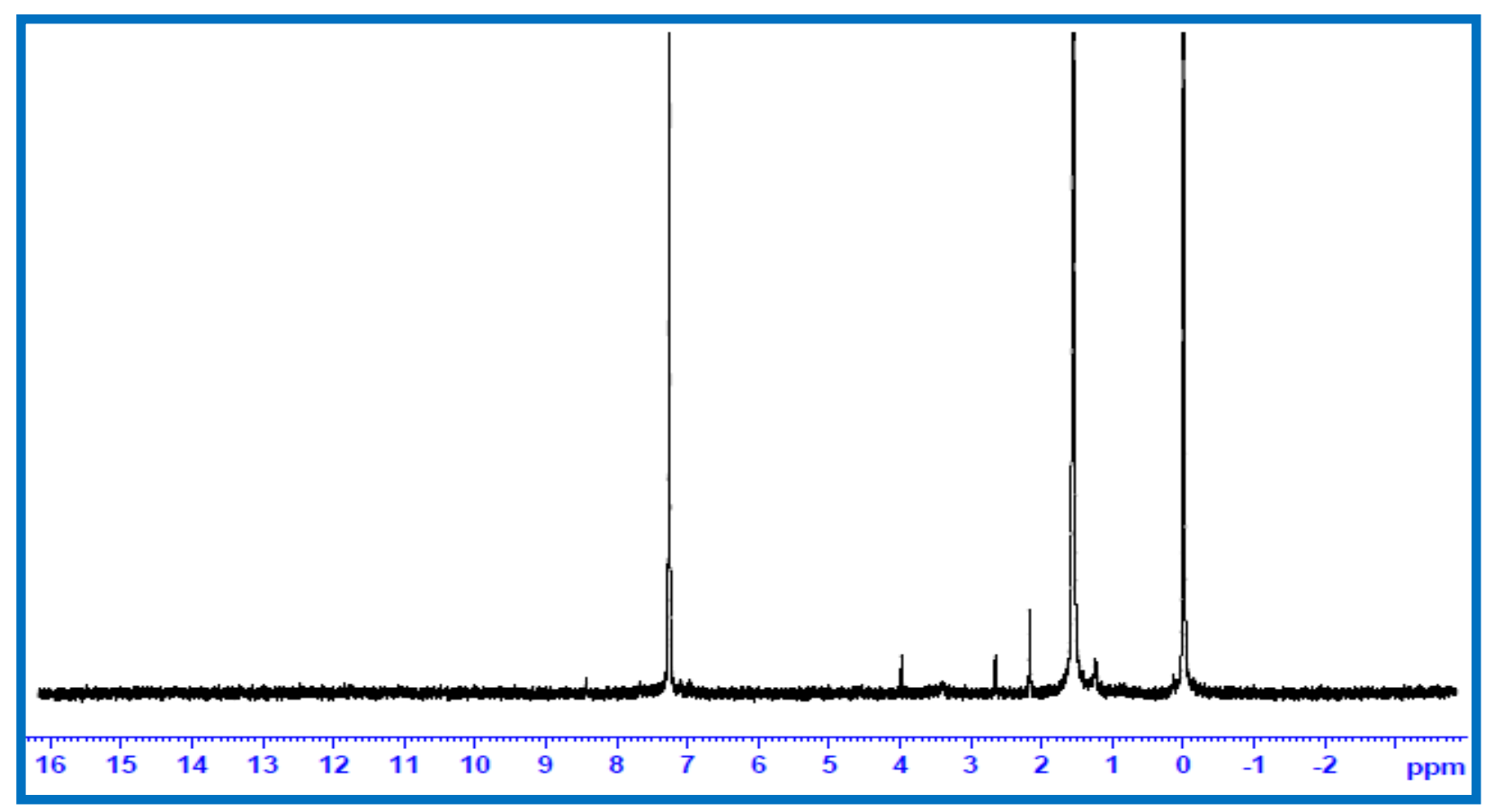

Figure S9. ${ }^{1} \mathrm{H}$ NMR spectrum of complex 3 in $\mathrm{CDCl}_{3}$

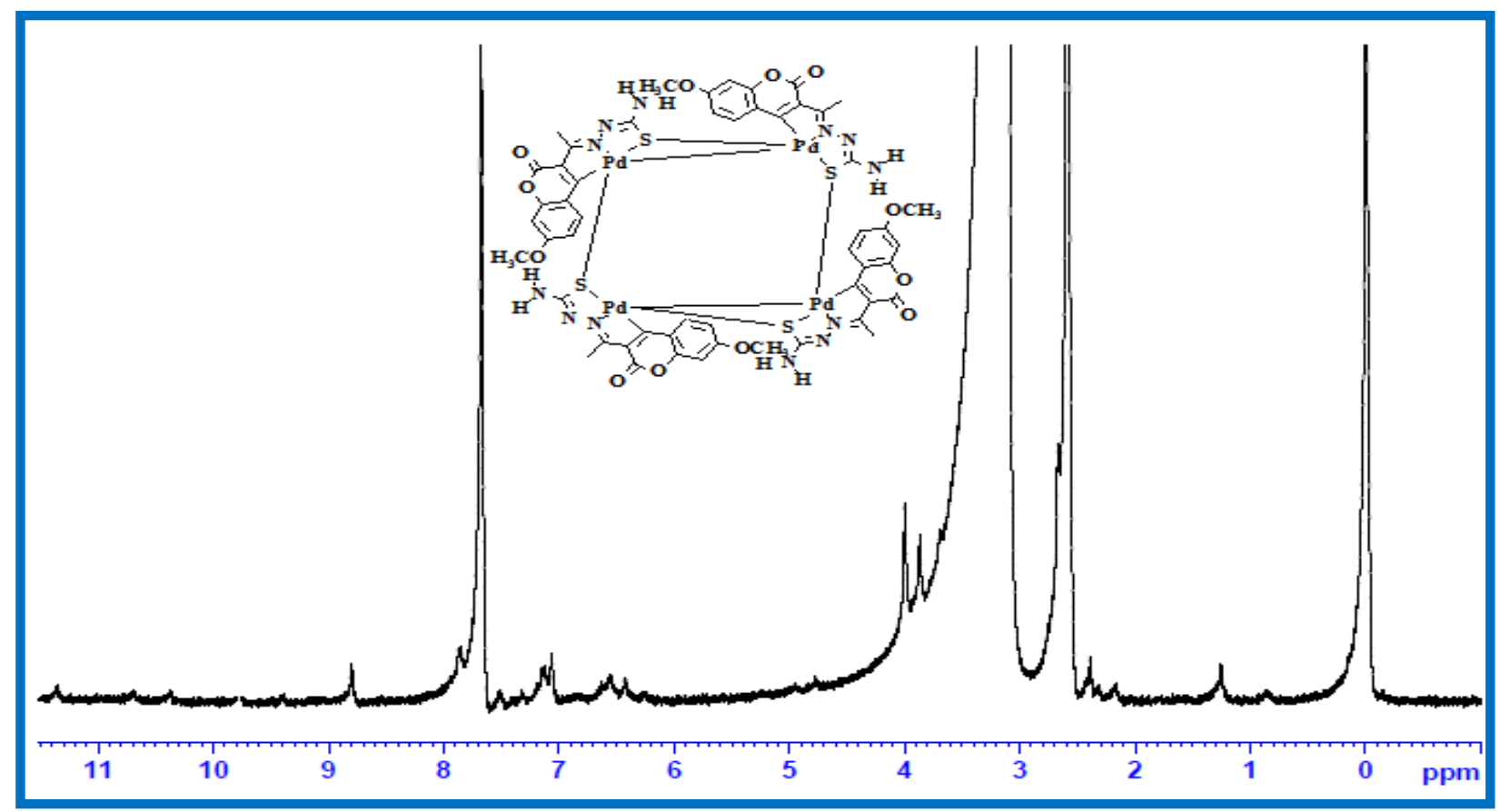

Figure S10. ${ }^{1} \mathrm{H}$ NMR spectrum of complex 1 in $\mathrm{CDCl}_{3}$ (with two drops of DMSO-d ) 


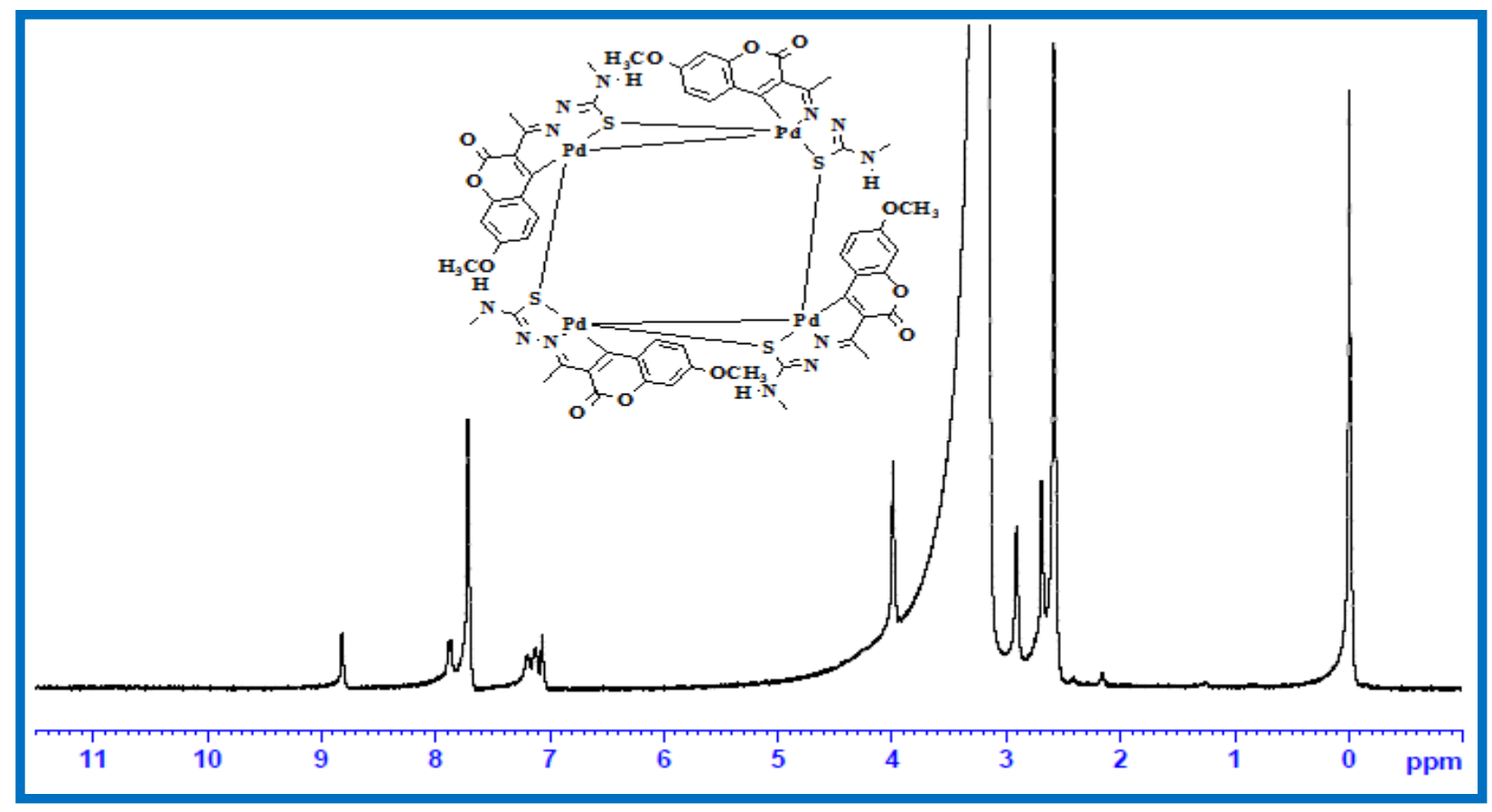

Figure S11. ${ }^{1} \mathrm{H}$ NMR spectrum of complex 2 in $\mathrm{CDCl}_{3}$ (with two drops of DMSO-d 6 )

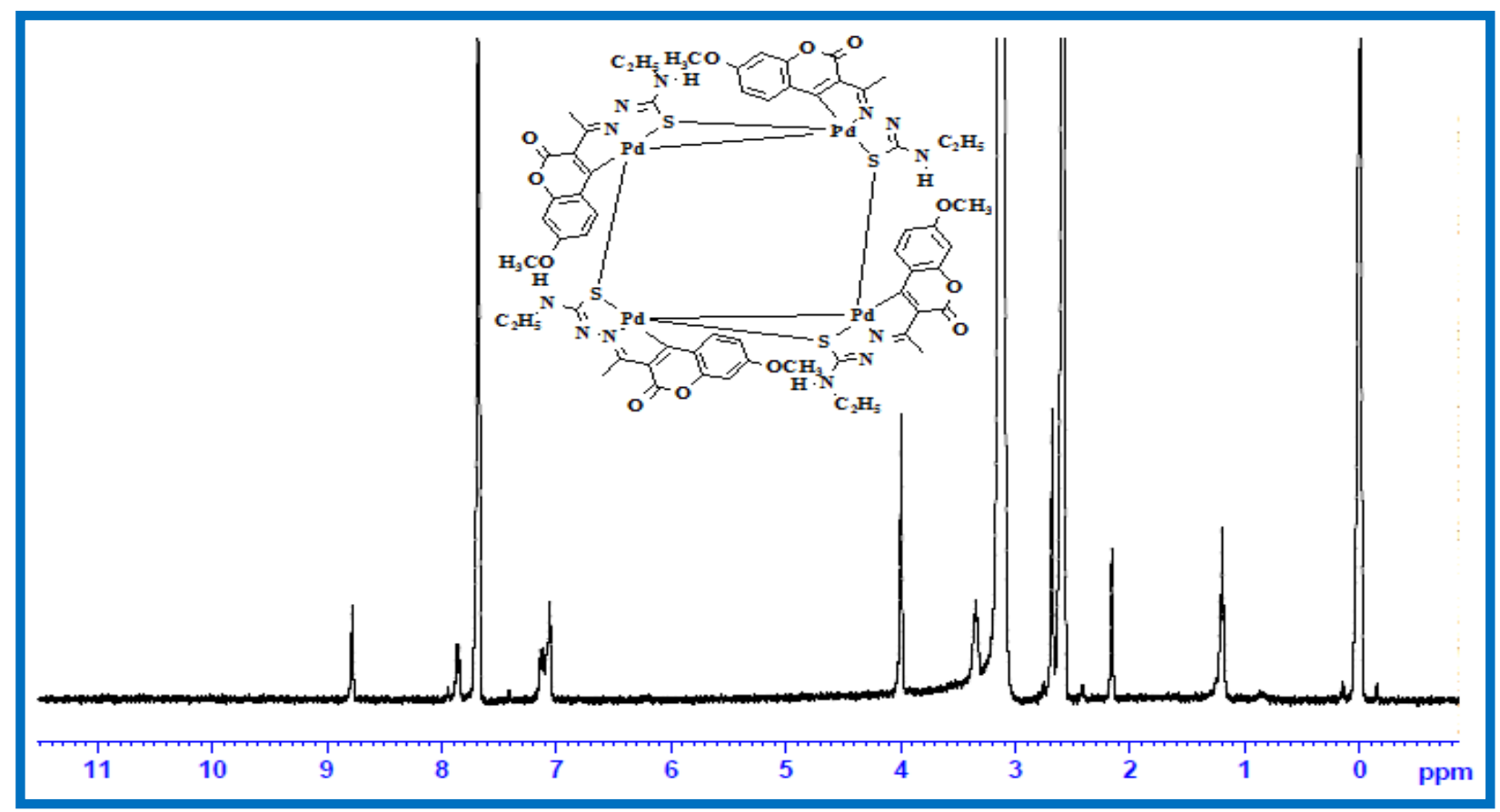

Figure S12. ${ }^{1} \mathrm{H}$ NMR spectrum of complex 3 in $\mathrm{CDCl}_{3}$ (with two drops of DMSO-d 6 ) 


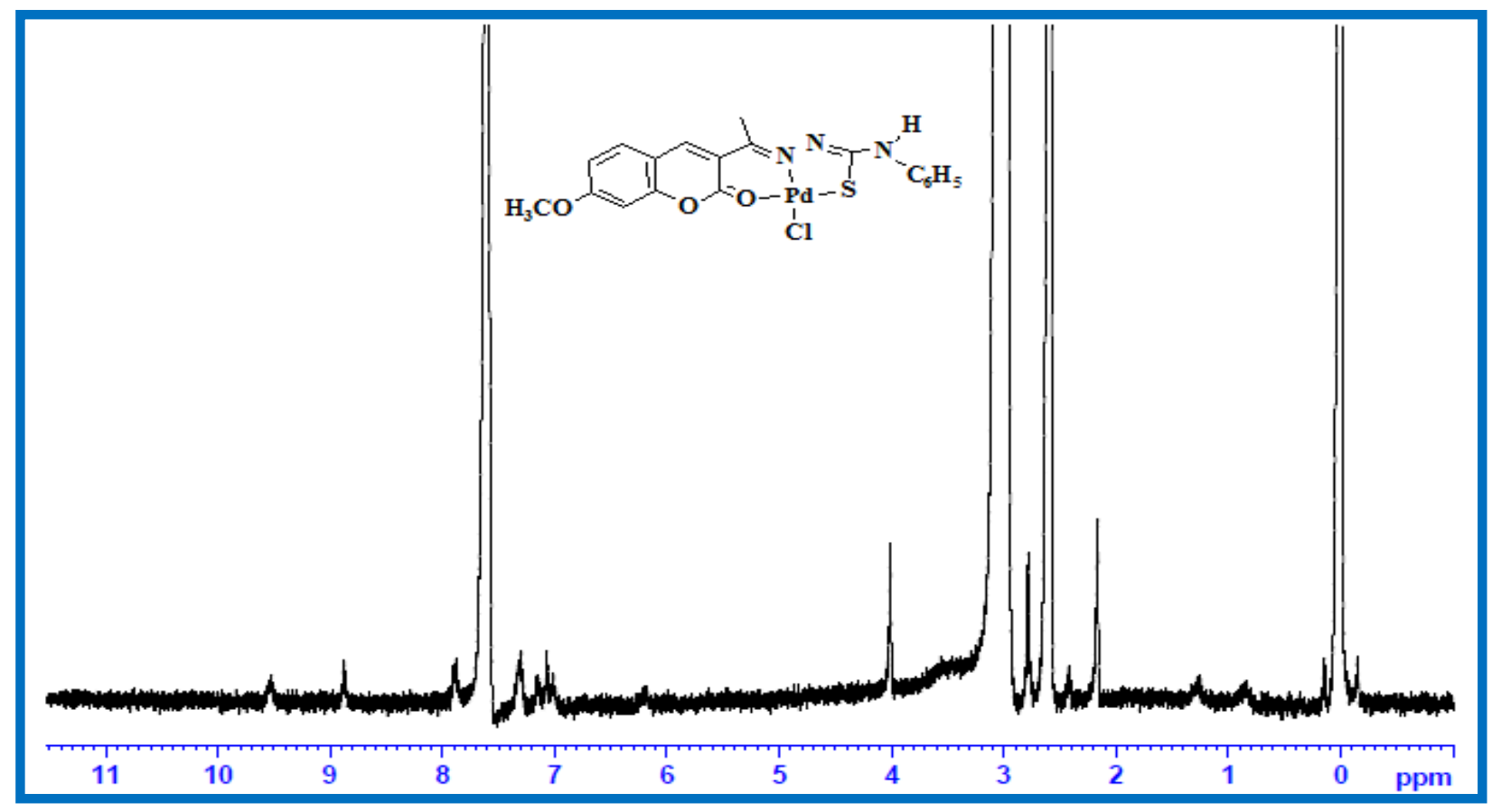

Figure S13. ${ }^{1} \mathrm{H}$ NMR spectrum of complex 4 in $\mathrm{CDCl}_{3}$ (with two drops of DMSO-d 6 )

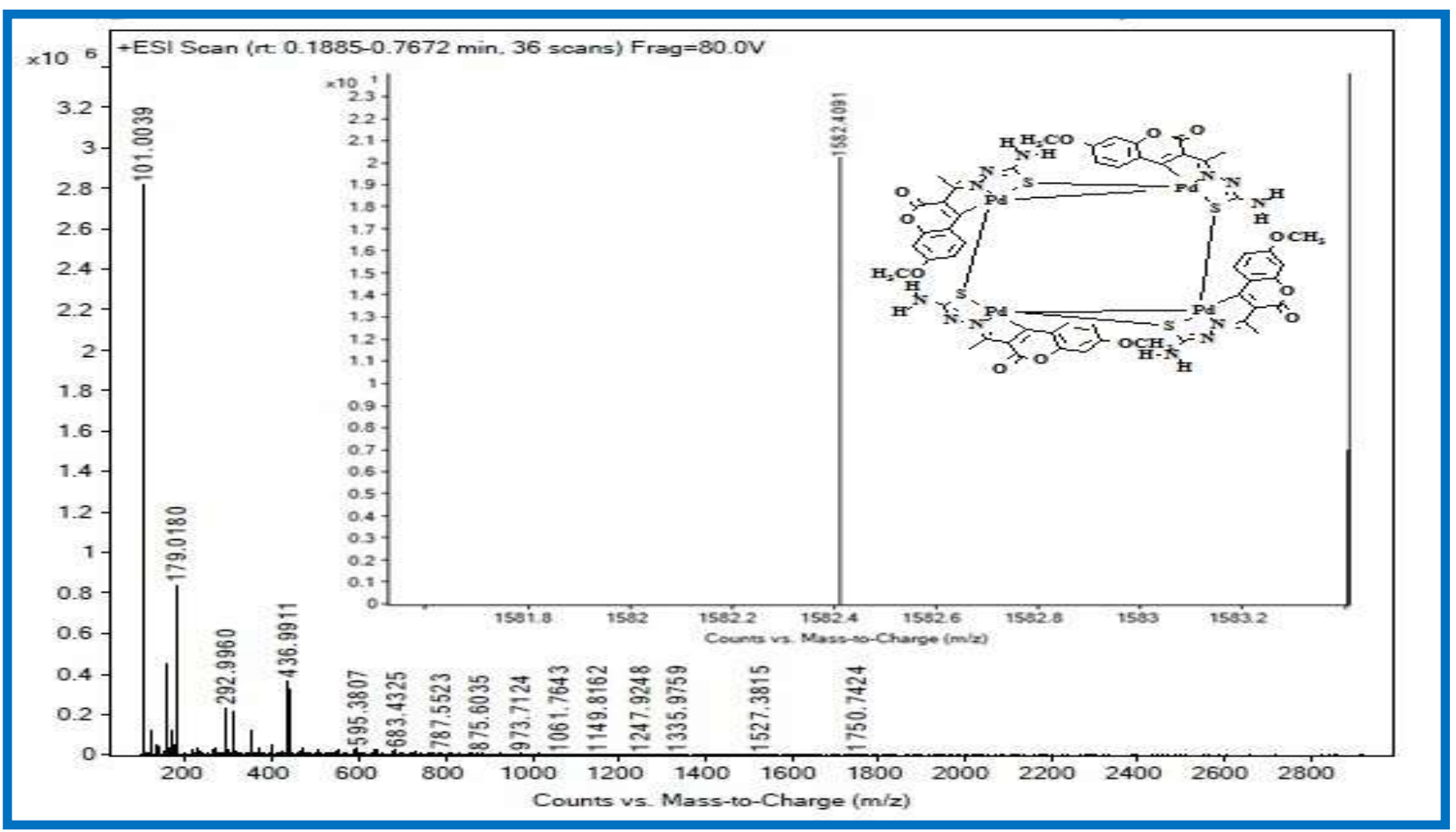

Figure S14. ESI MS spectrum of complex 1 


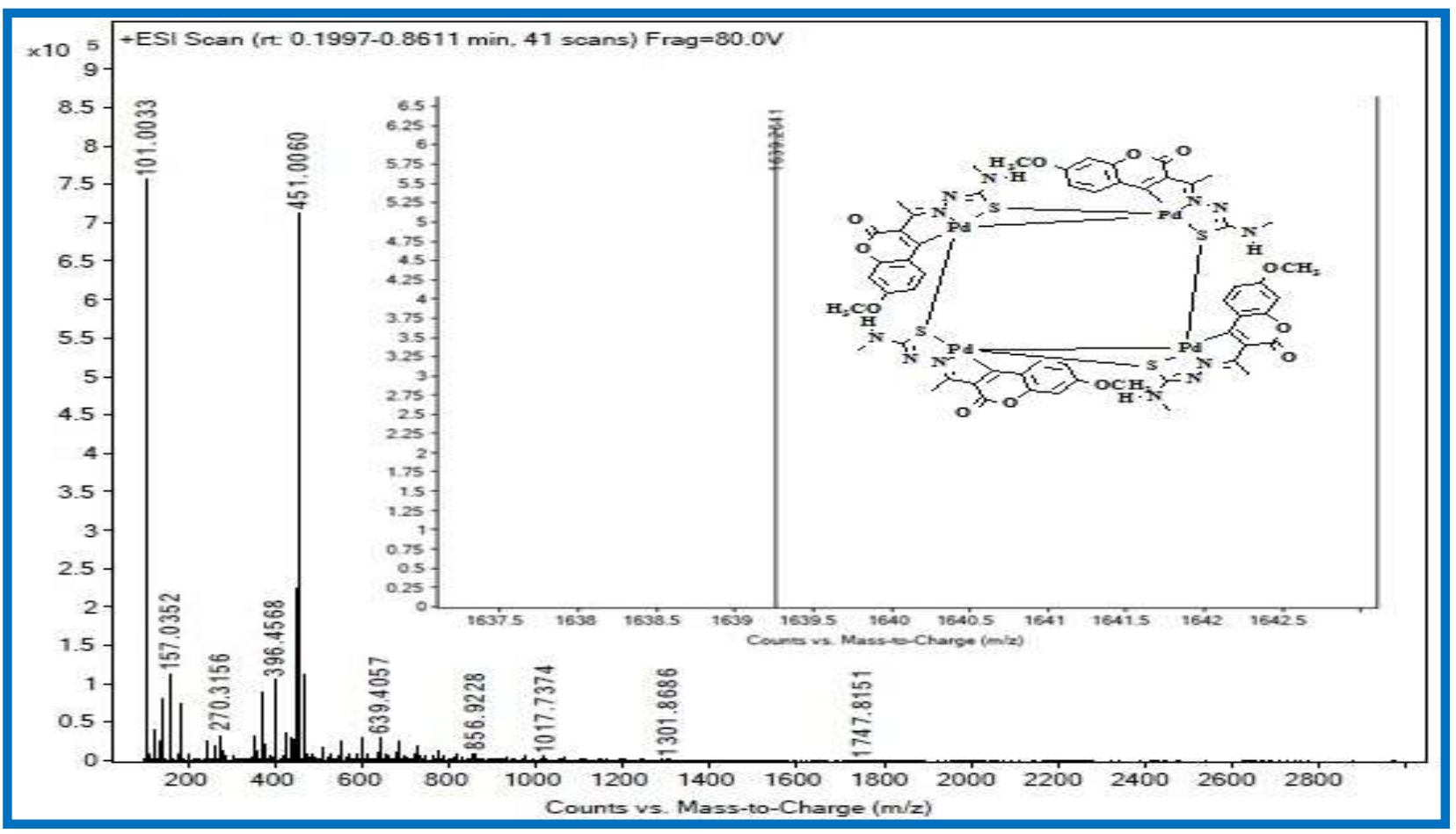

Figure S15. ESI MS spectrum of complex 2

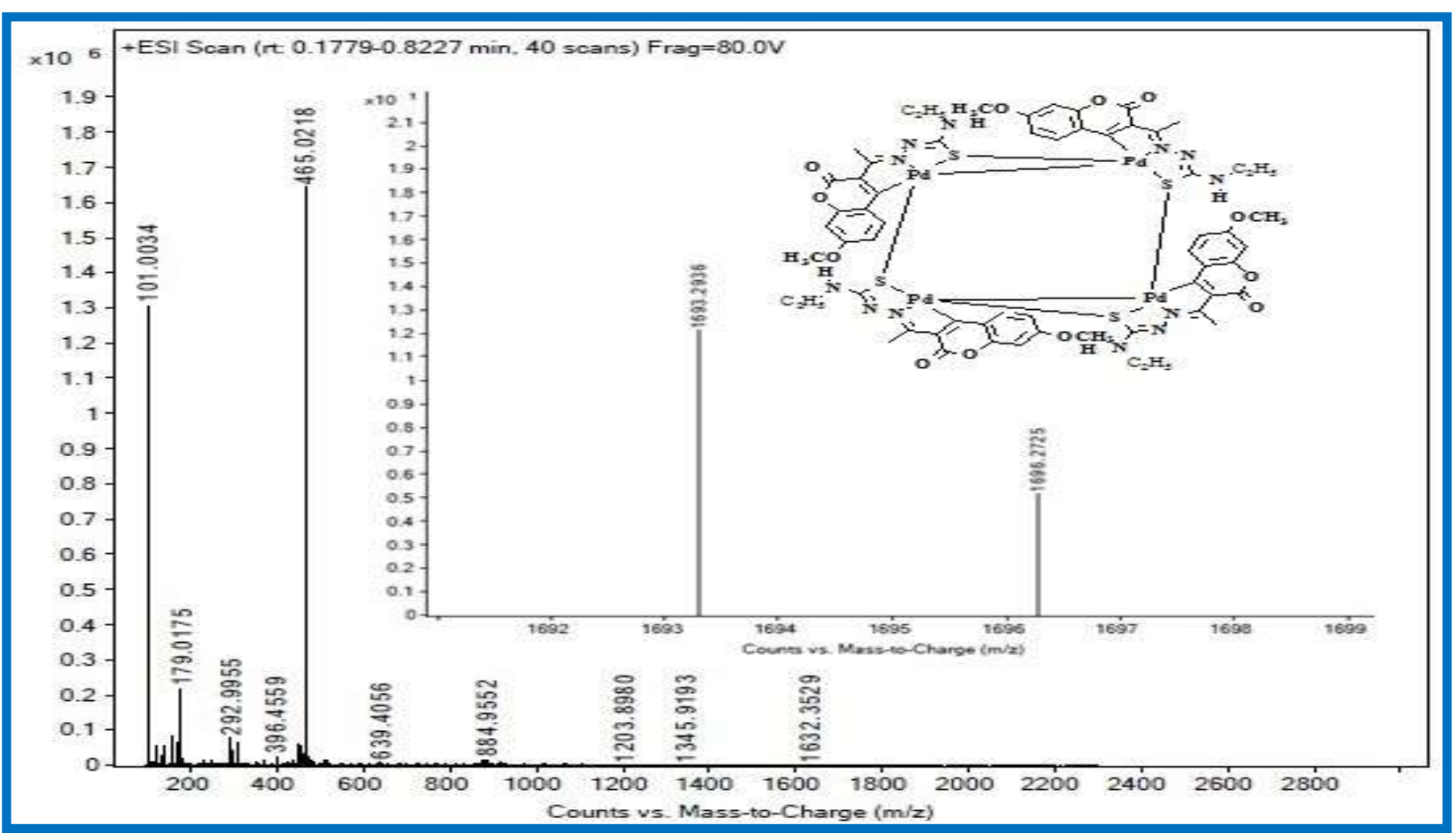

Figure S16. ESI MS spectrum of complex 3 


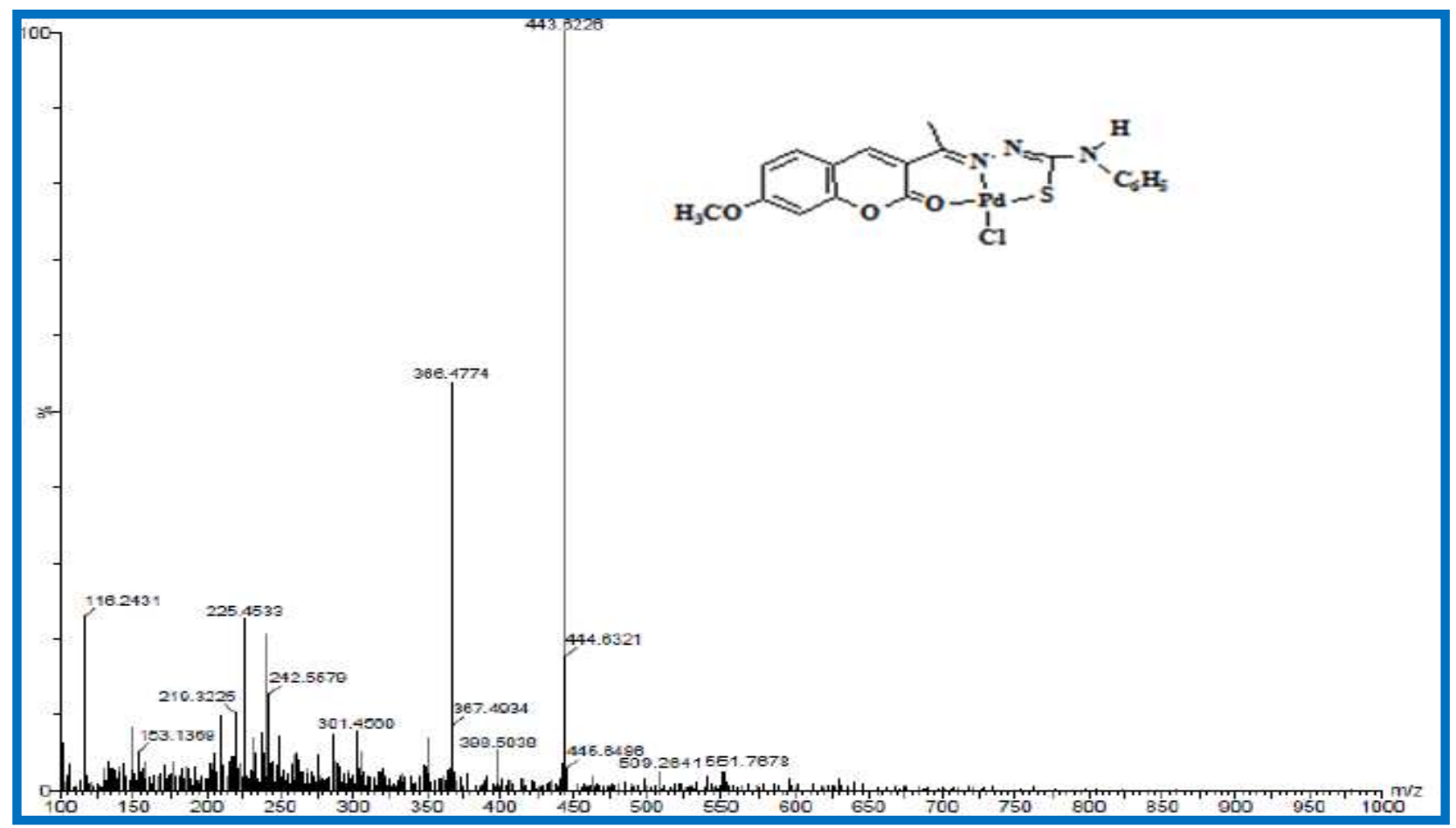

Figure S17. ESI MS spectrum of complex 4

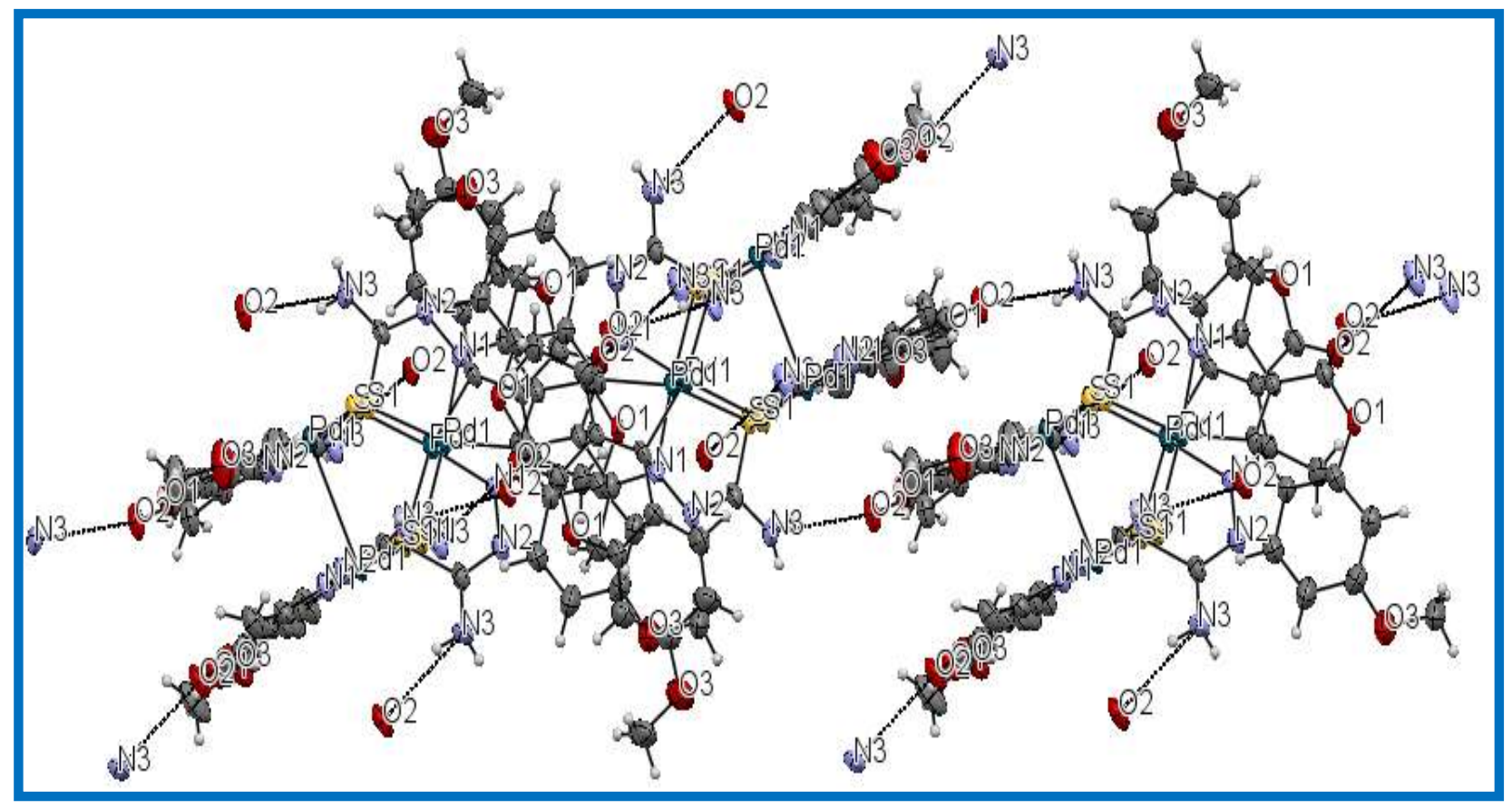

Figure S18. ORTEP diagram of the complex 1 with hydrogen bonding 


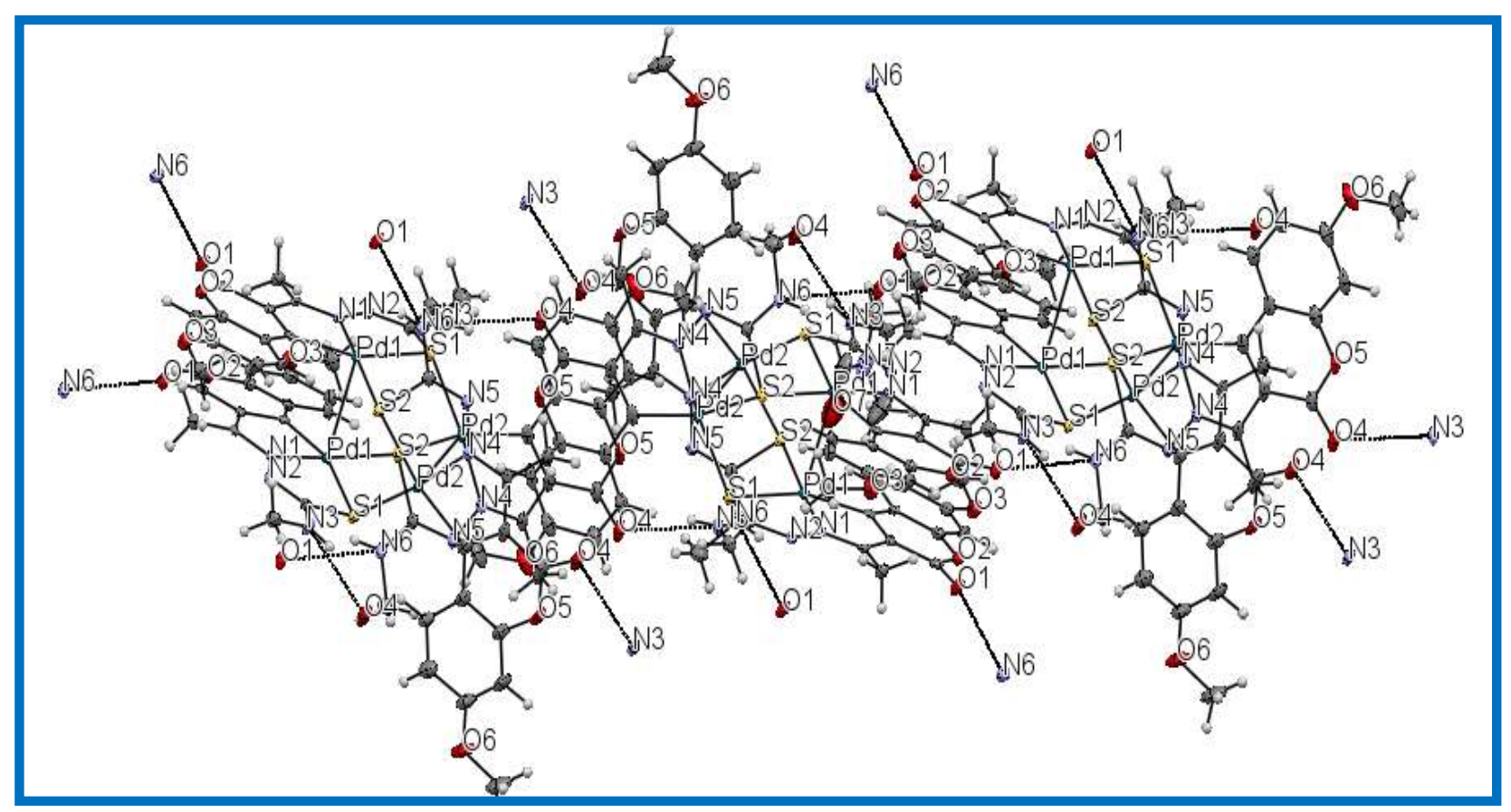

Figure S19. ORTEP diagram of the complex 2 with hydrogen bonding

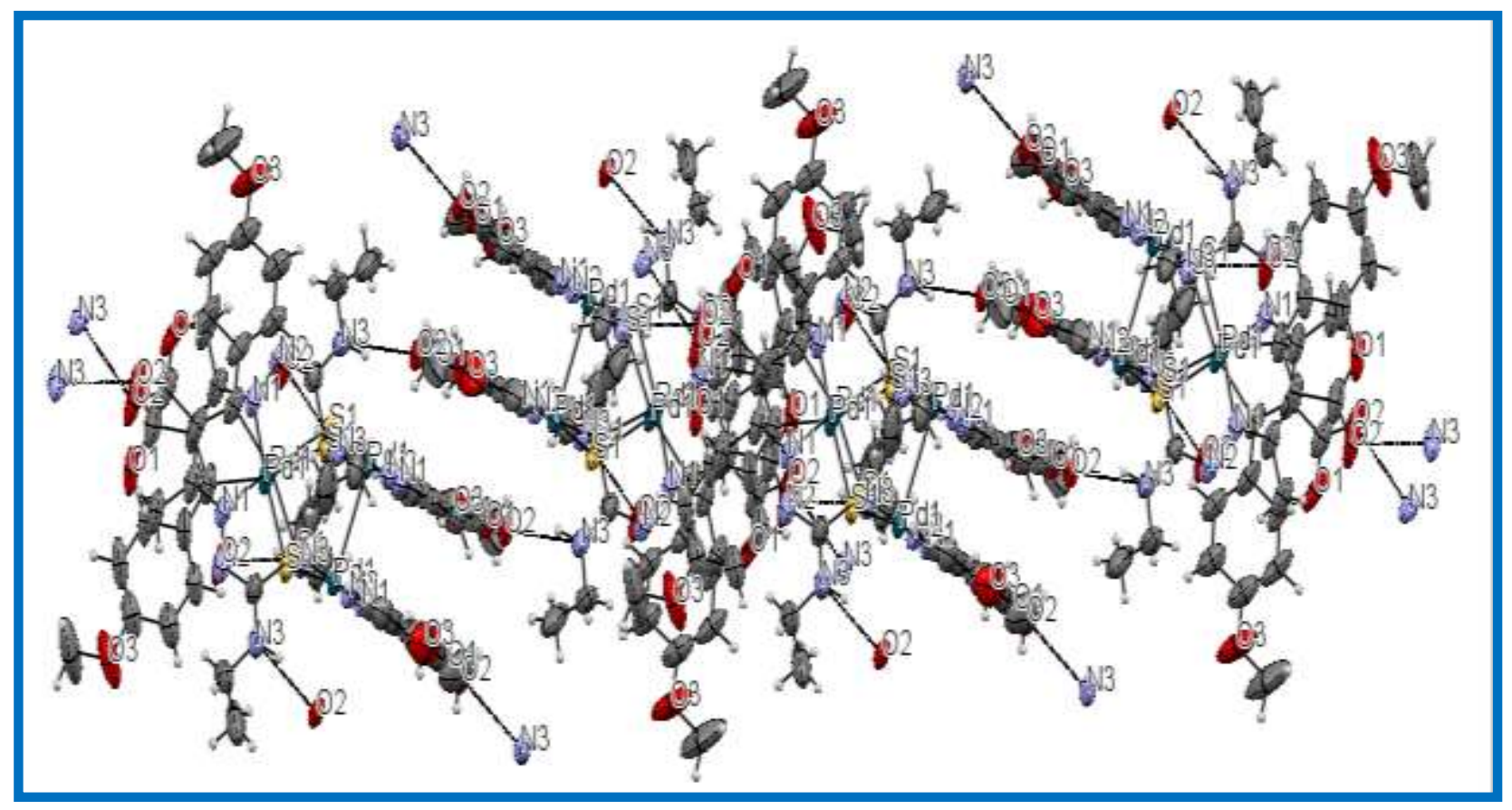

Figure S20. ORTEP diagram of the complex 3 with hydrogen bonding 


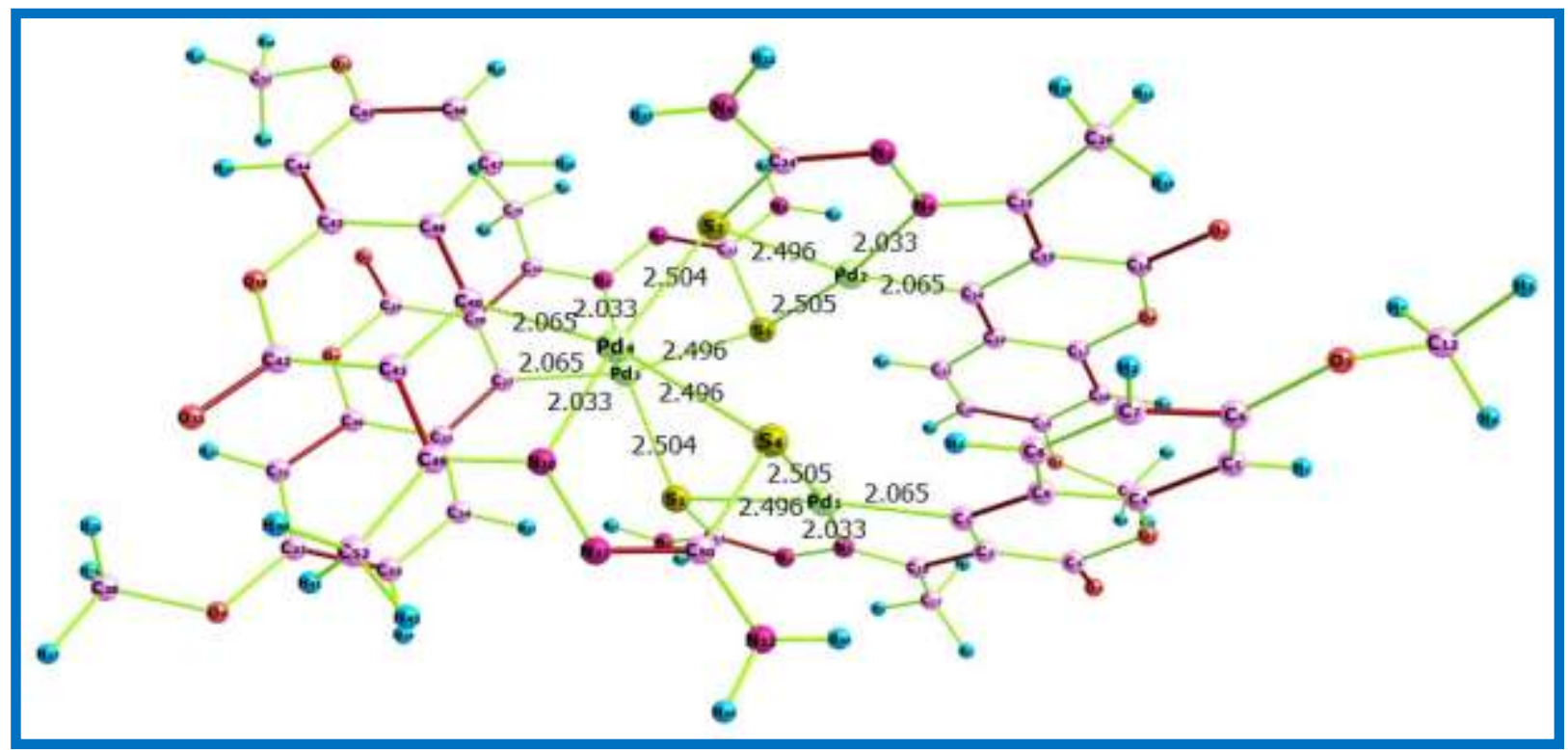

Figure S21. Optimized structure of complex 1

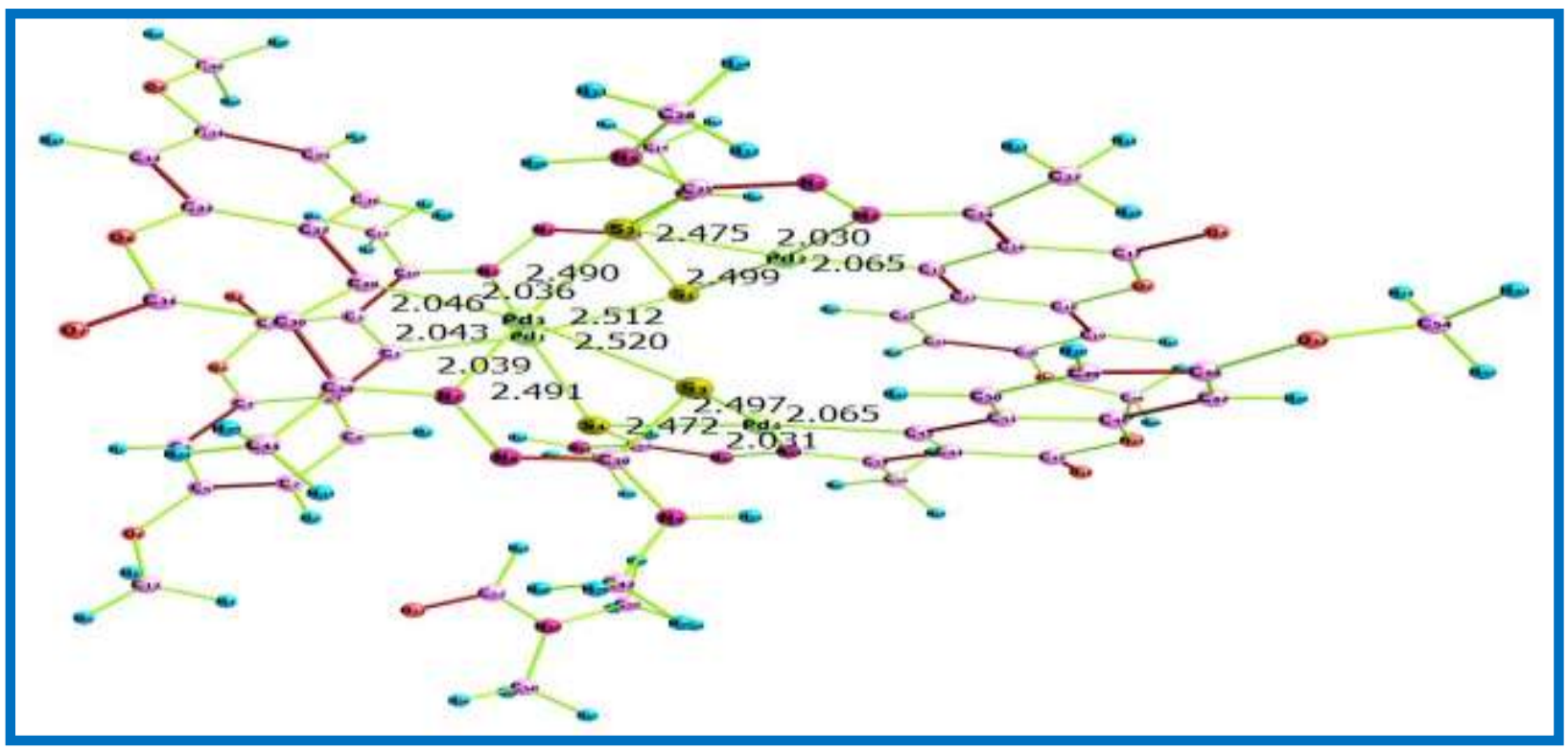

Figure S22. Optimized structure of complex 2 


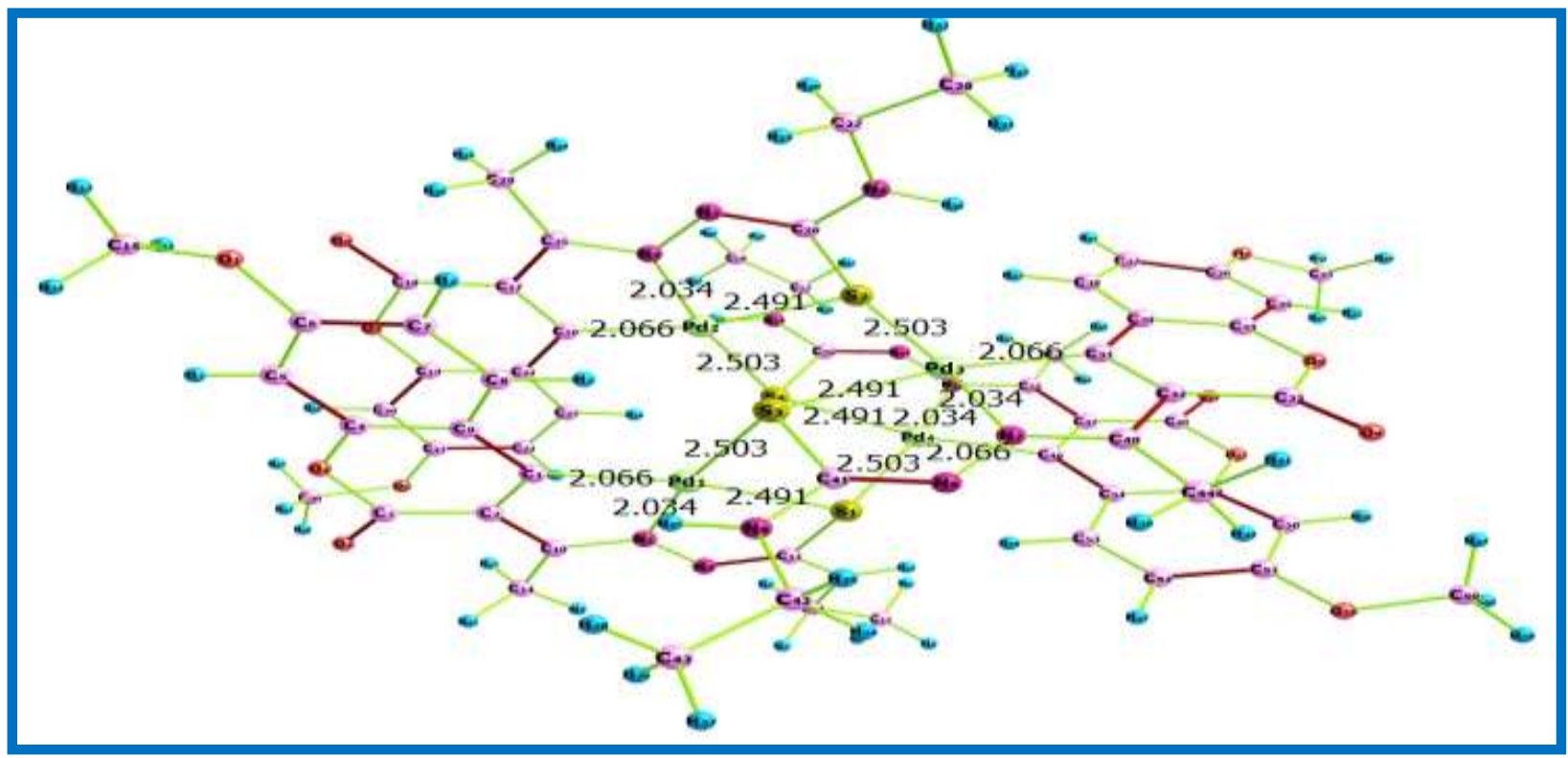

Figure S23. Optimized structure of complex 3

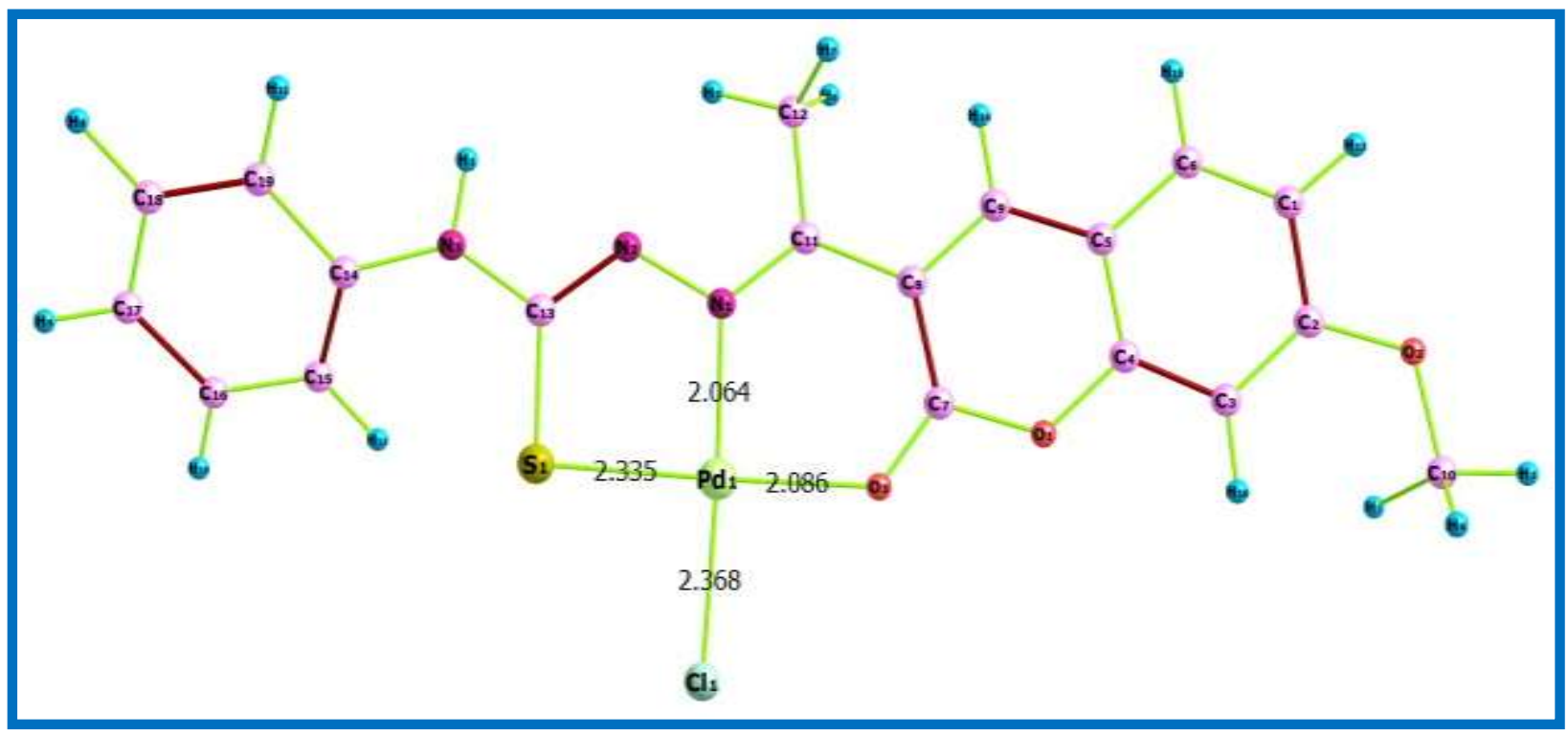

Figure S24. Optimized structure of complex 4 


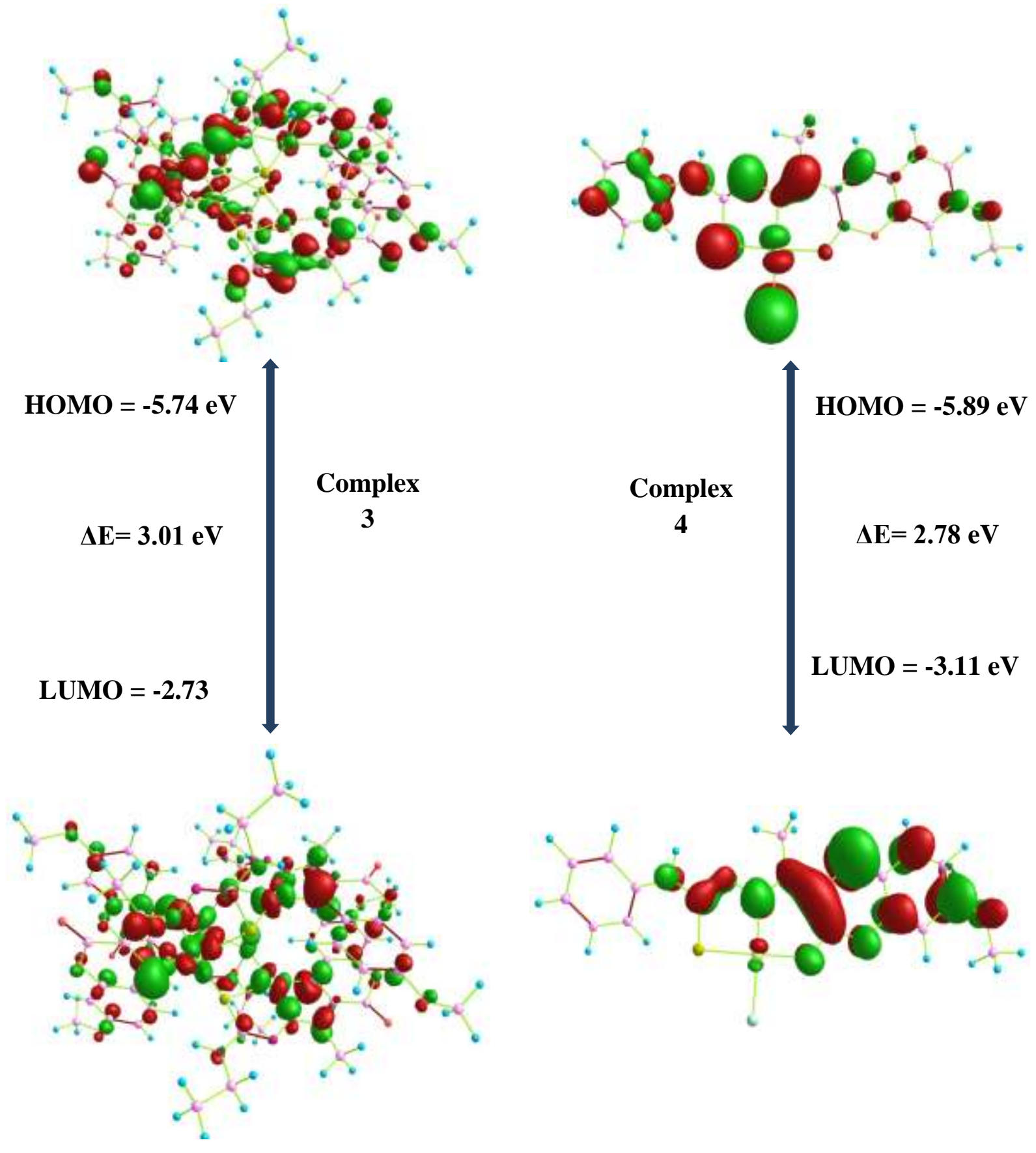

Figure S25. Energy level diagram between HUMO and LUMO of complexes 3 and 4 


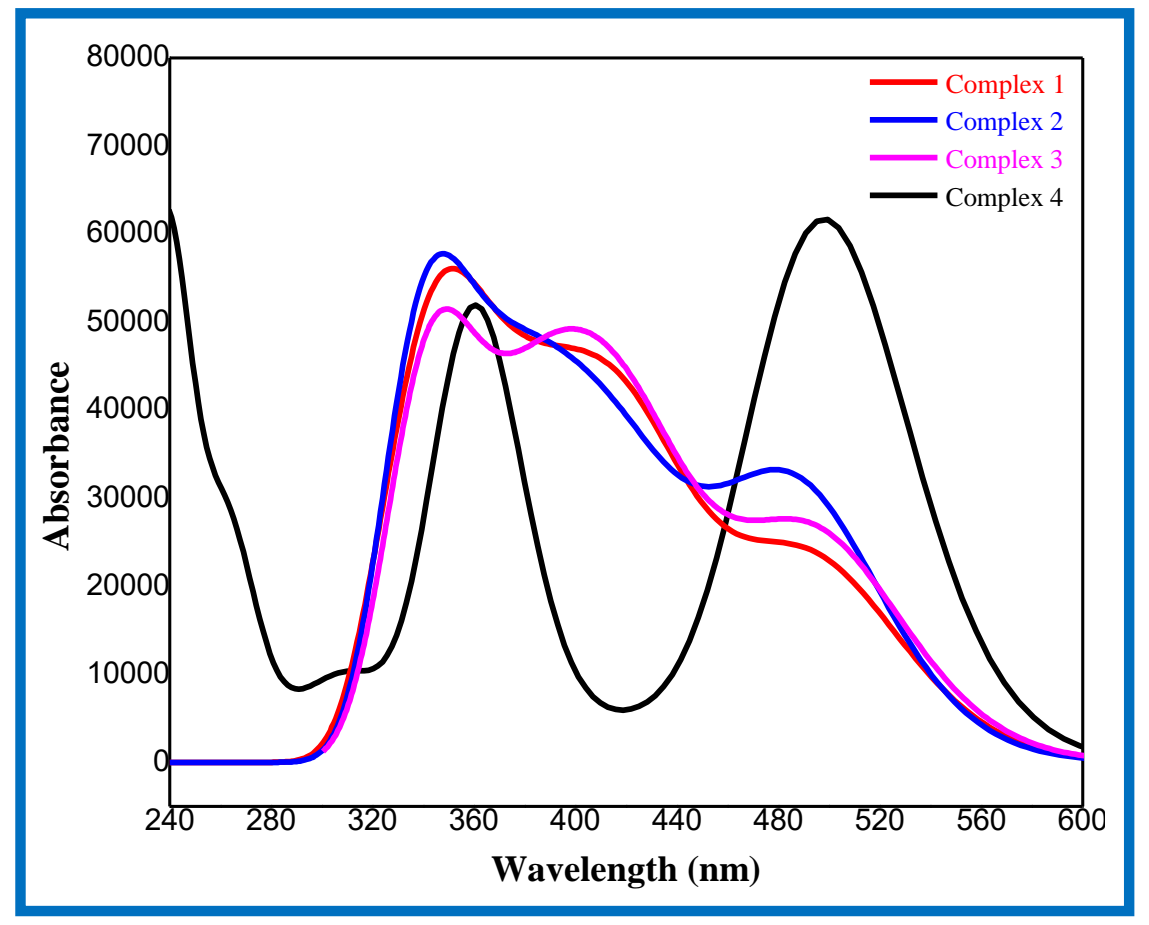

Figure S26. Electronic spectra of complexes 1-4 calculated by FMO analysis

\section{GCMS}

\section{Table 1, Entry 1}

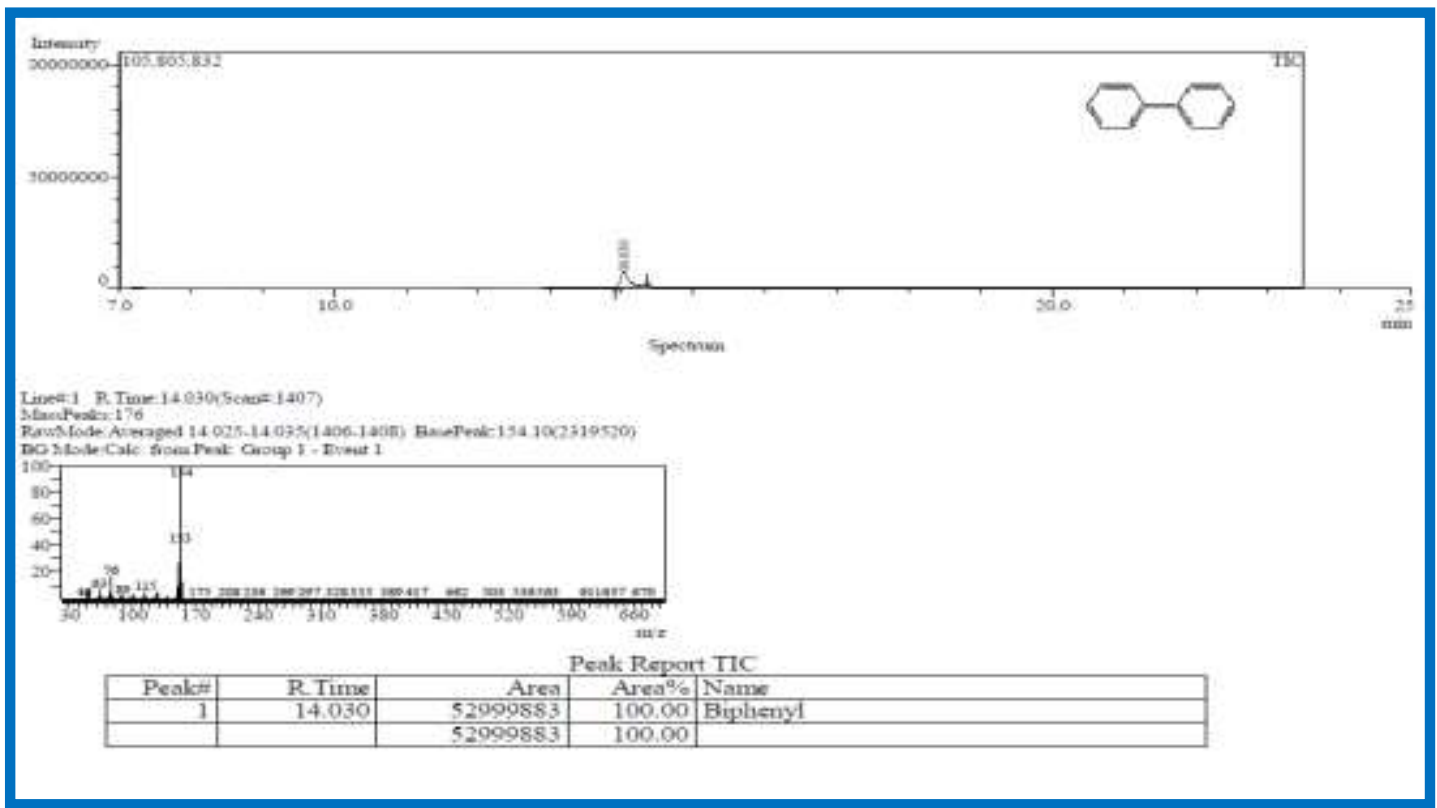

Figure S27. GCMS of biphenyl 
2. Table 1, Entry 4

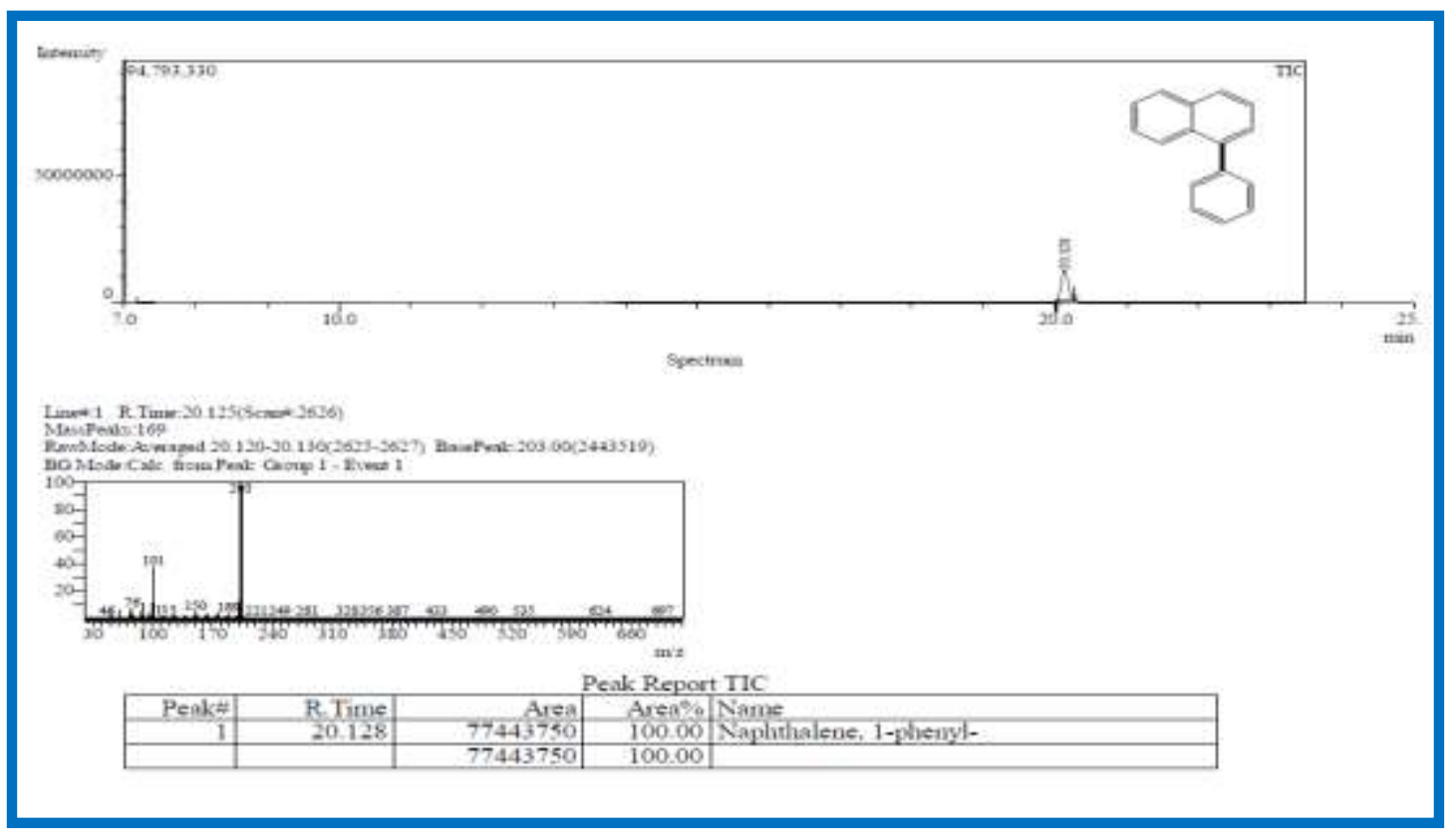

Figure S28. GCMS of 1-phenyl naphthalene

3. Table 1, Entry 5

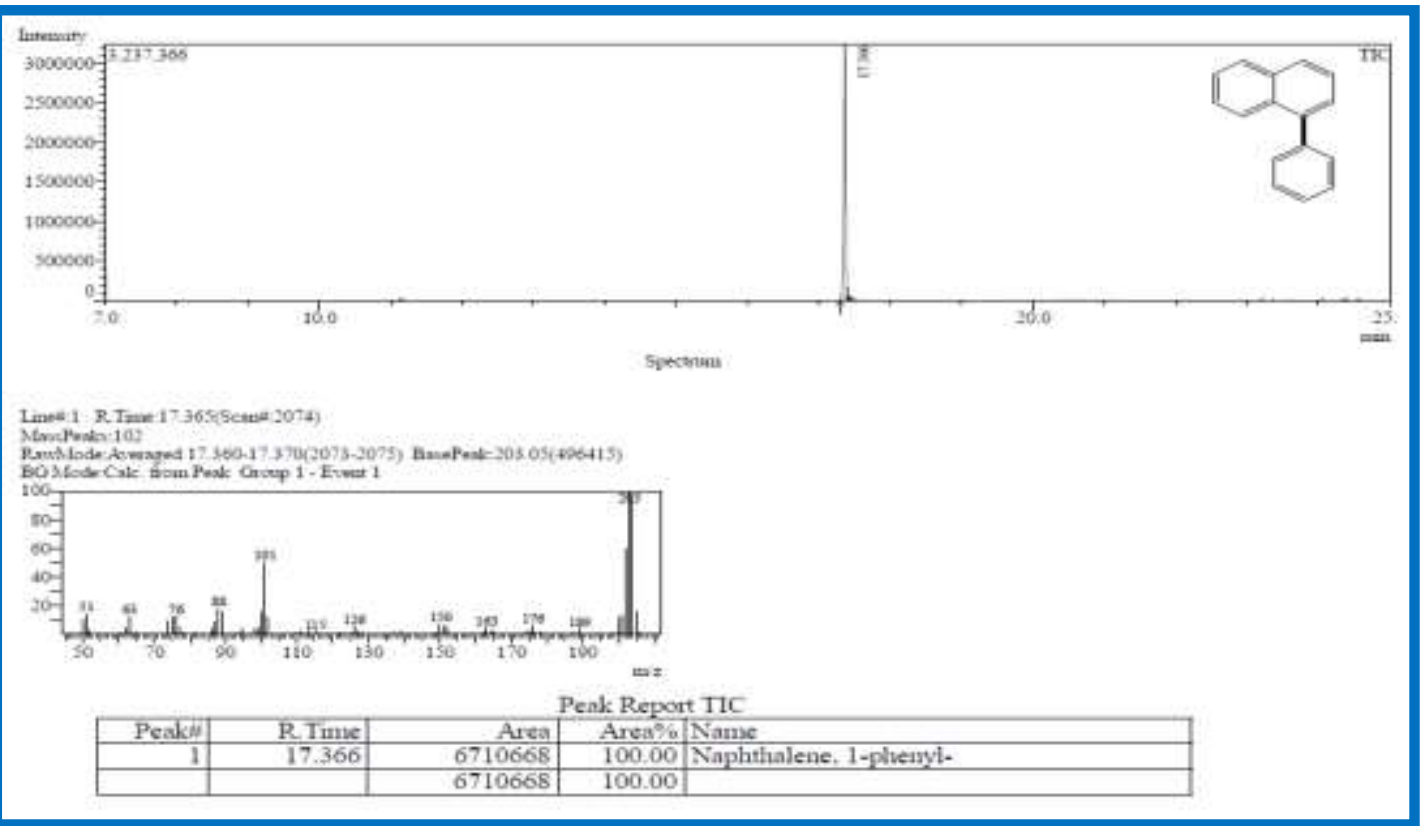

Figure S29. GCMS of 1-phenyl naphthalene 


\section{Table 1, Entry 6}

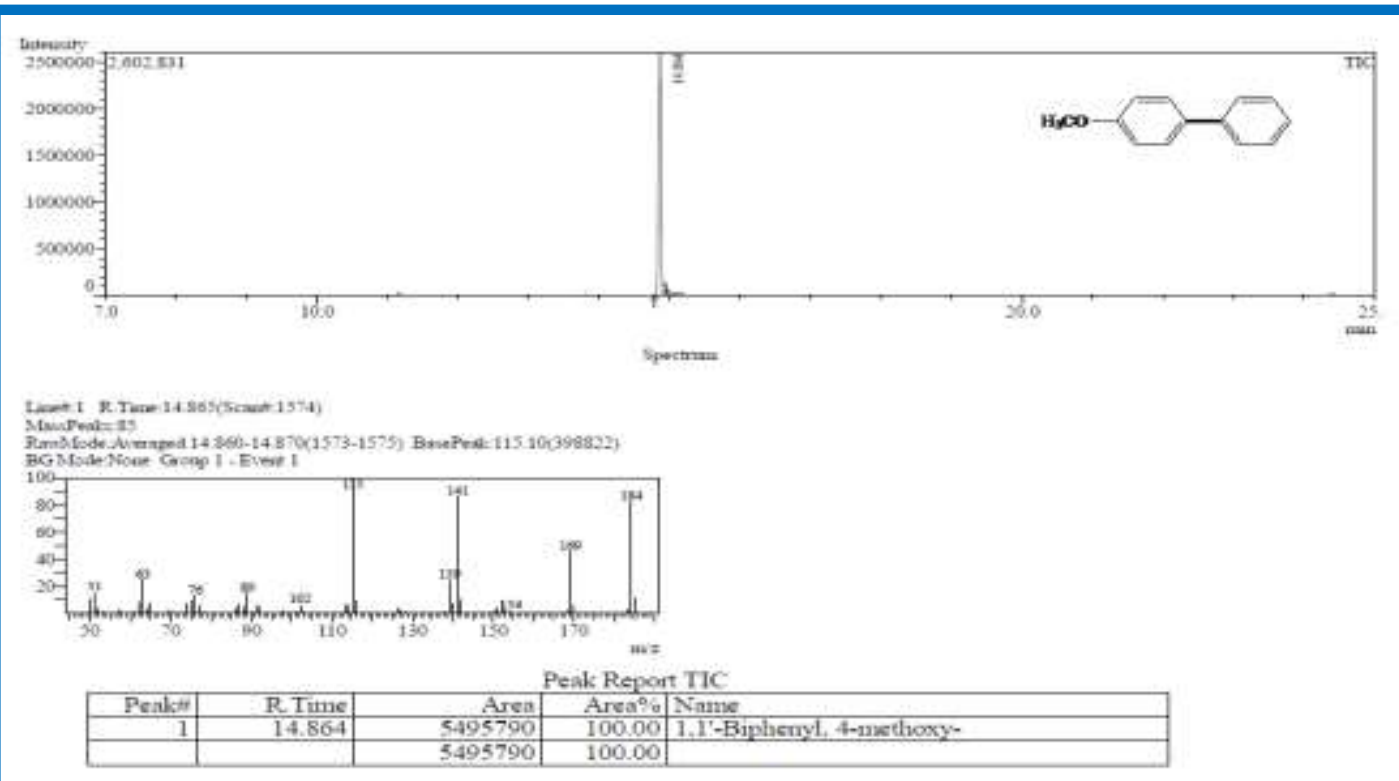

Figure S30. GCMS of 4-phenyl anisole

5. Table 1, Entry 8

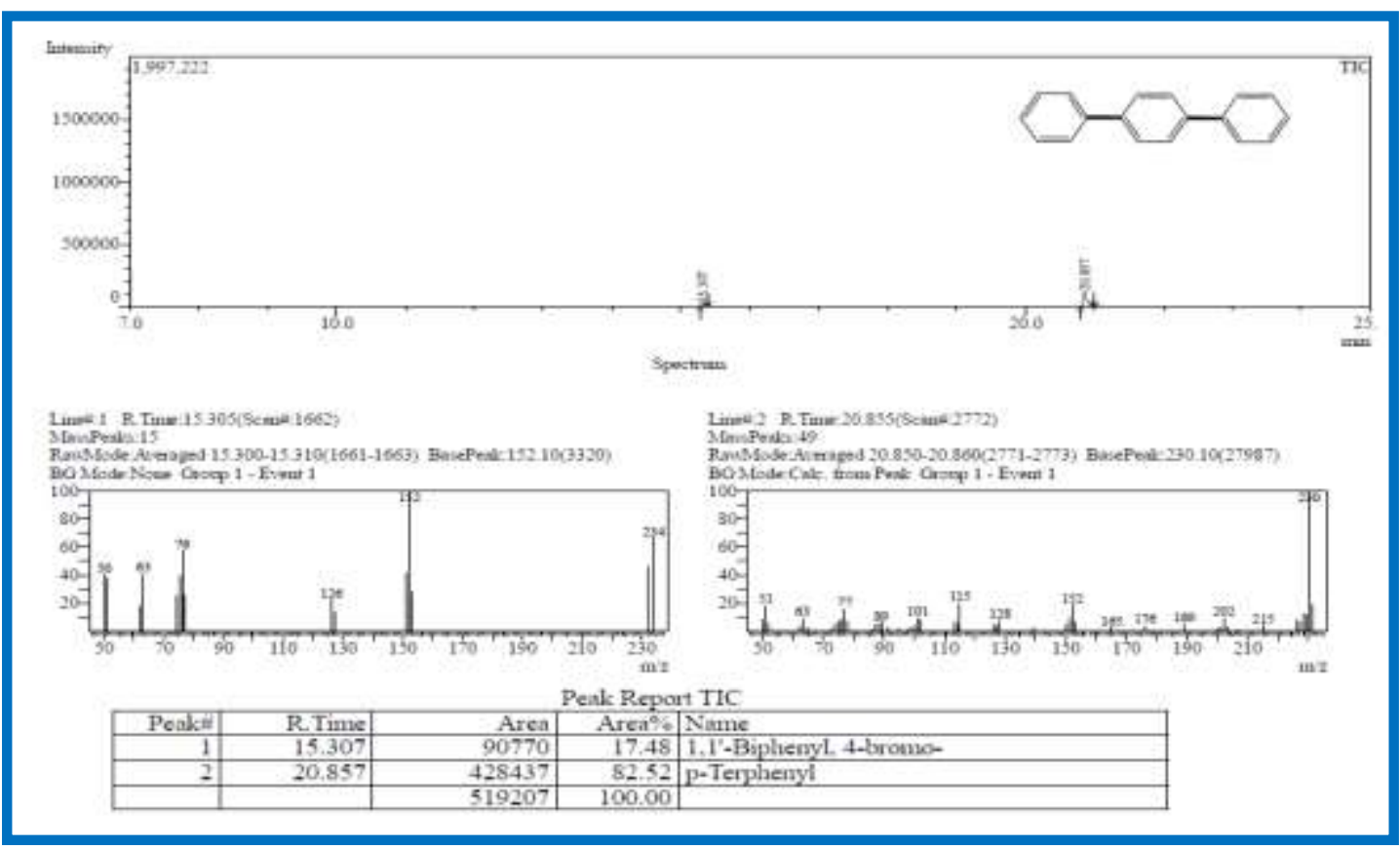

Figure S31. GCMS of Terphenyl 
6. Table 1, Entry 9

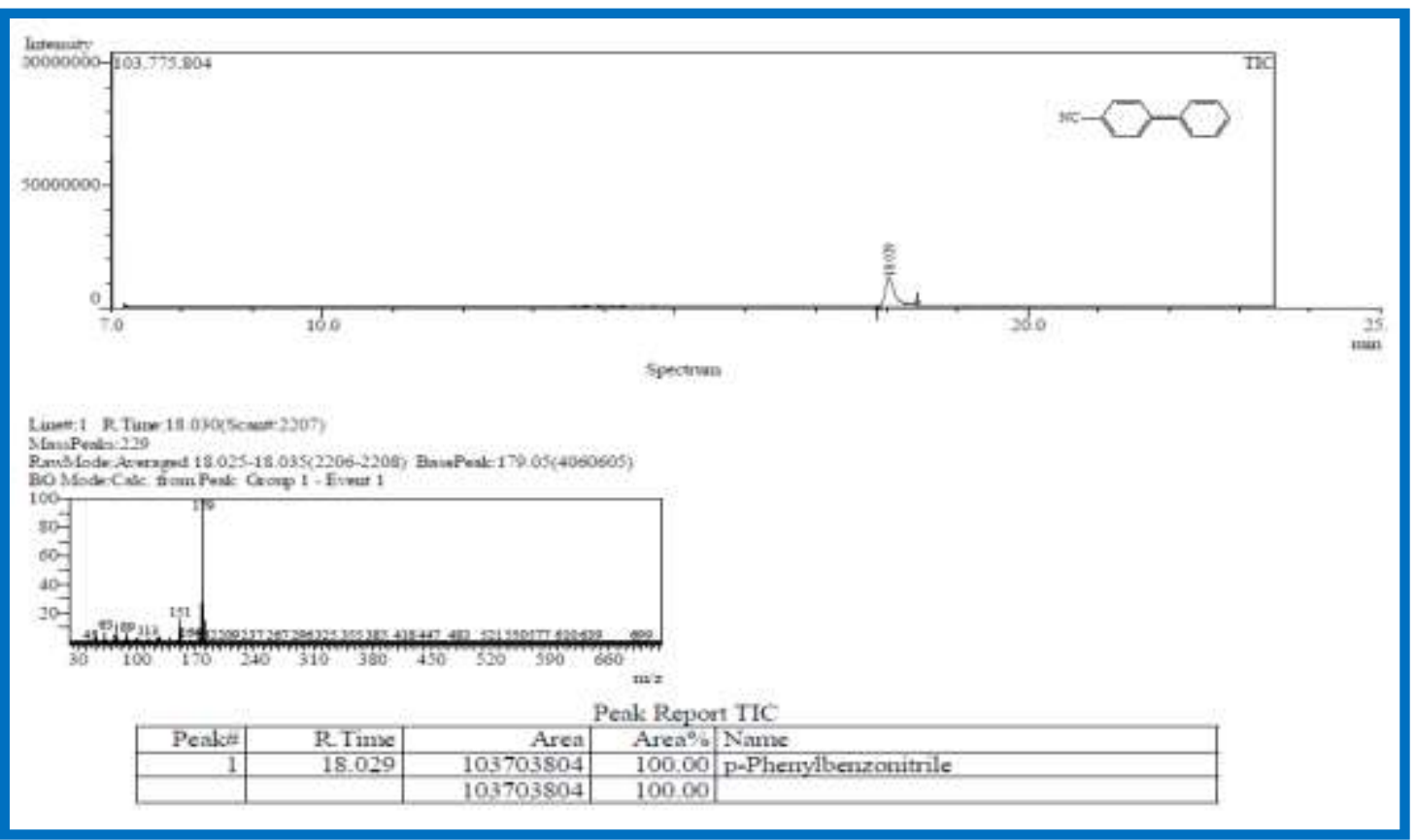

Figure S32. GCMS of 4-phenyl benzonitrile

7. Table 1 , Entry 12

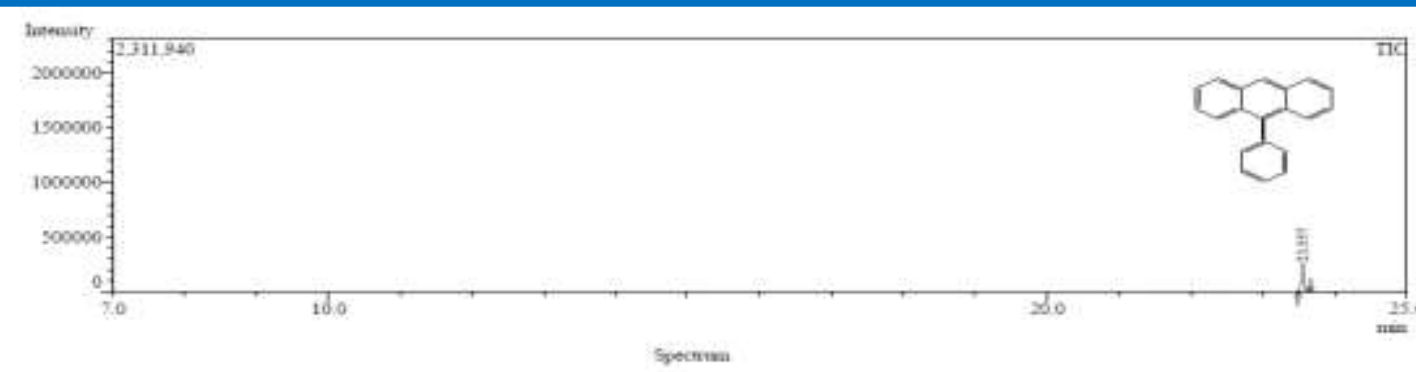

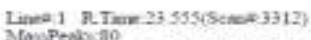

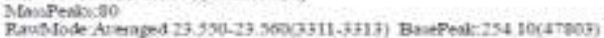

BG Mode.cal Sam Peil Goup I- Everit 1

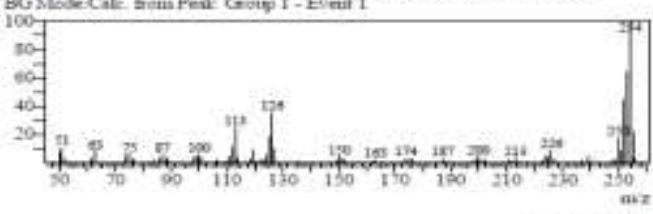

Peak Report TIC

\begin{tabular}{|r|r|r|r|l|}
\hline Peake & RTime & Area & Area & Name \\
\hline 1 & 23.557 & 693893 & 100,00 & Anthracene, 9-phenyl- \\
\hline & & 693893 & 100,00 & \\
\hline
\end{tabular}

Figure S33. GCMS of 9-phenyl anthracene 
8. Table 1, Entry 14

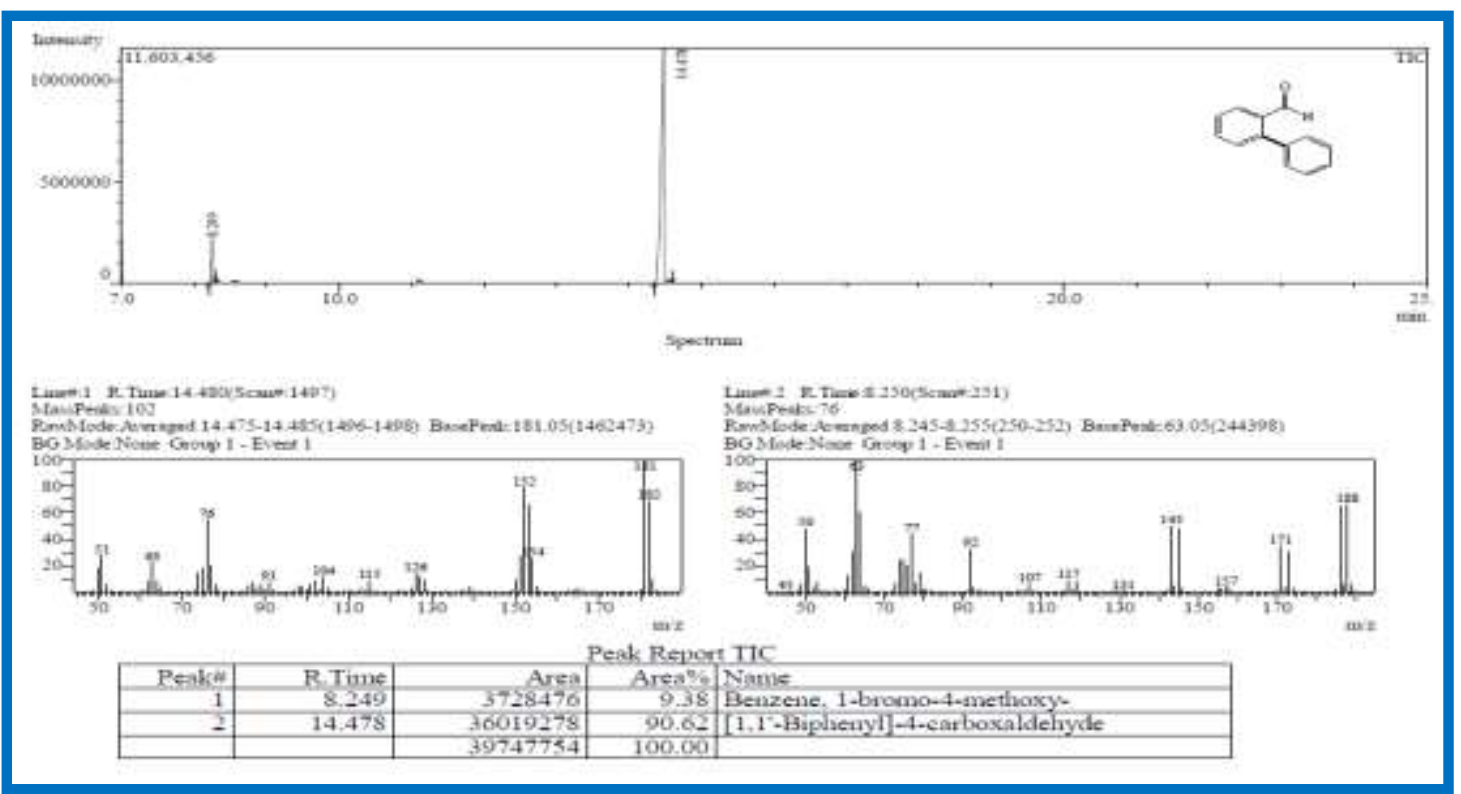

Figure S34. GCMS of 2-phenyl benzaldehyde 


\section{Table 1, Entry 20}

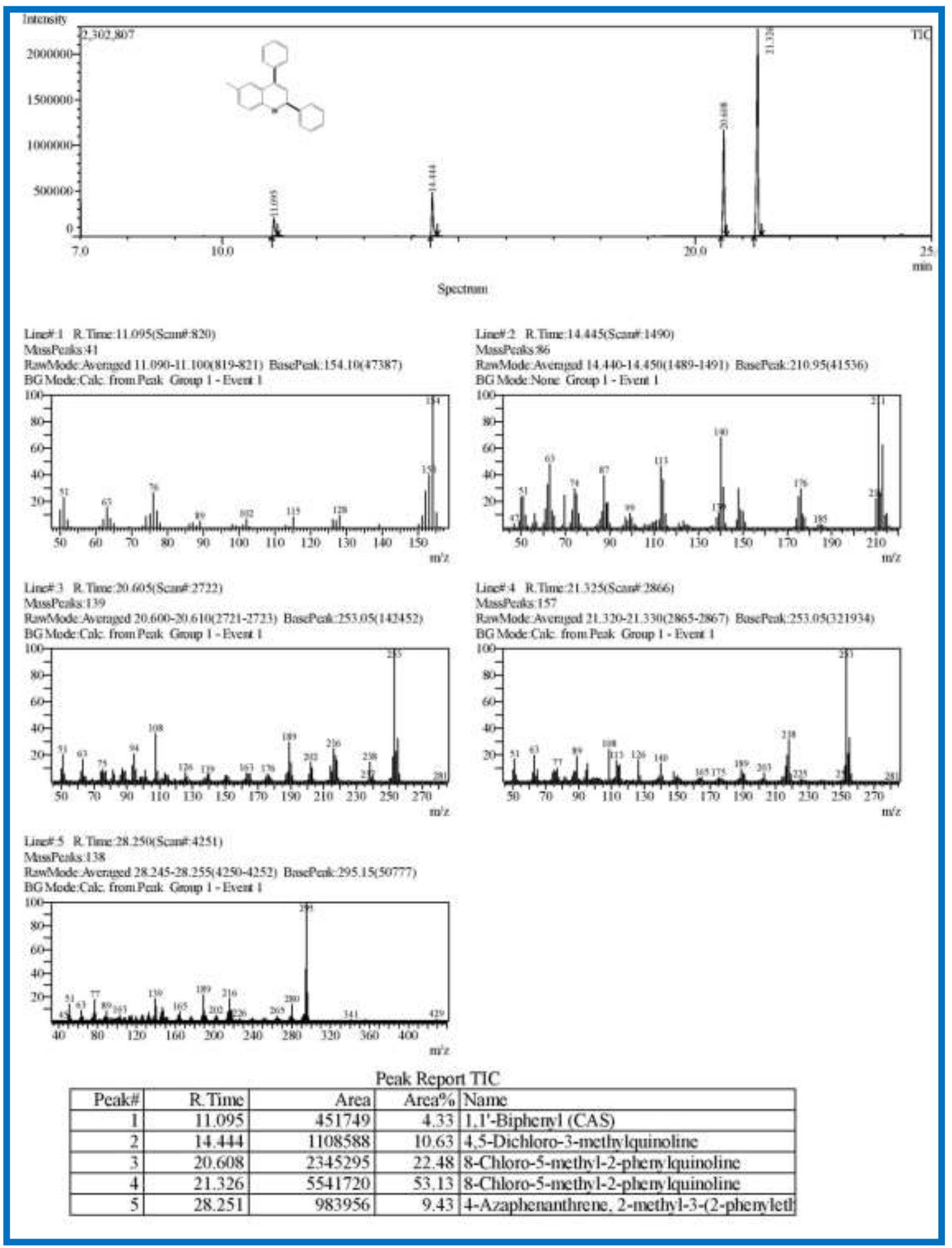

Figure S35. GCMS of 2,4-diphenyl-6-methylquinoline 
1. Table 1, Entry 10

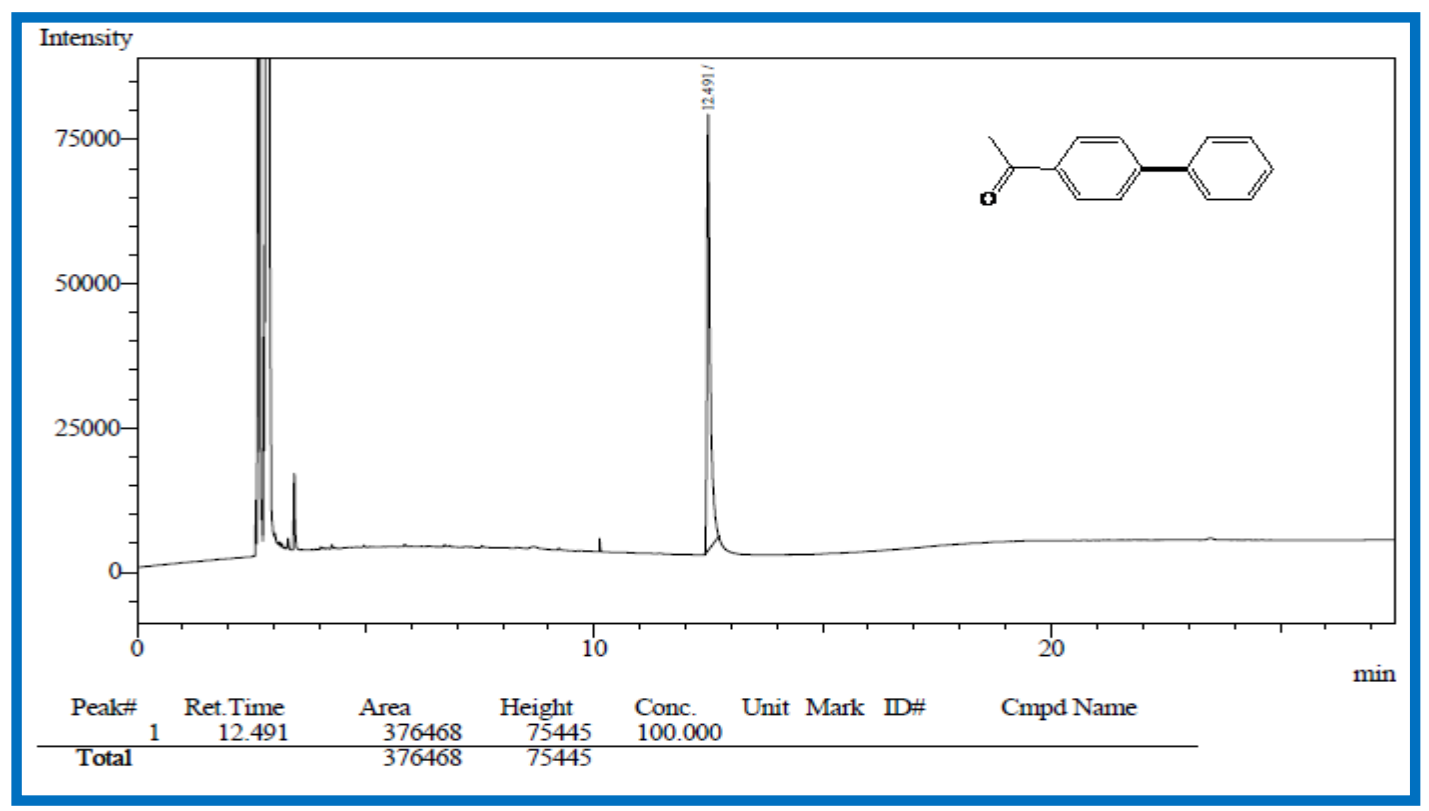

Figure S36. GC of 4-phenylacetophenone

2. Table 1, Entry 11

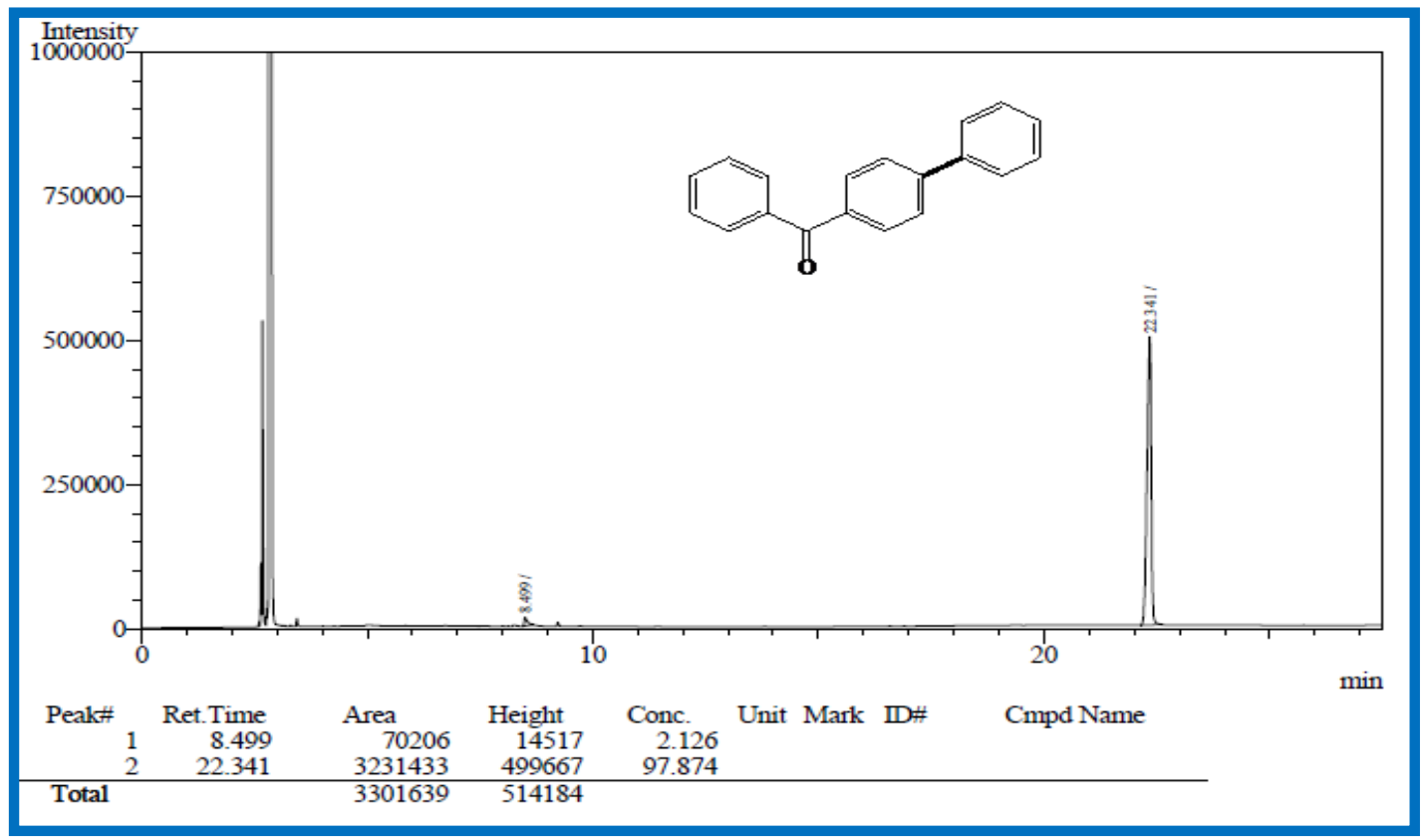

Figure S37. GC of 4-phenylbenzophenone 
3. Table 1, Entry 13

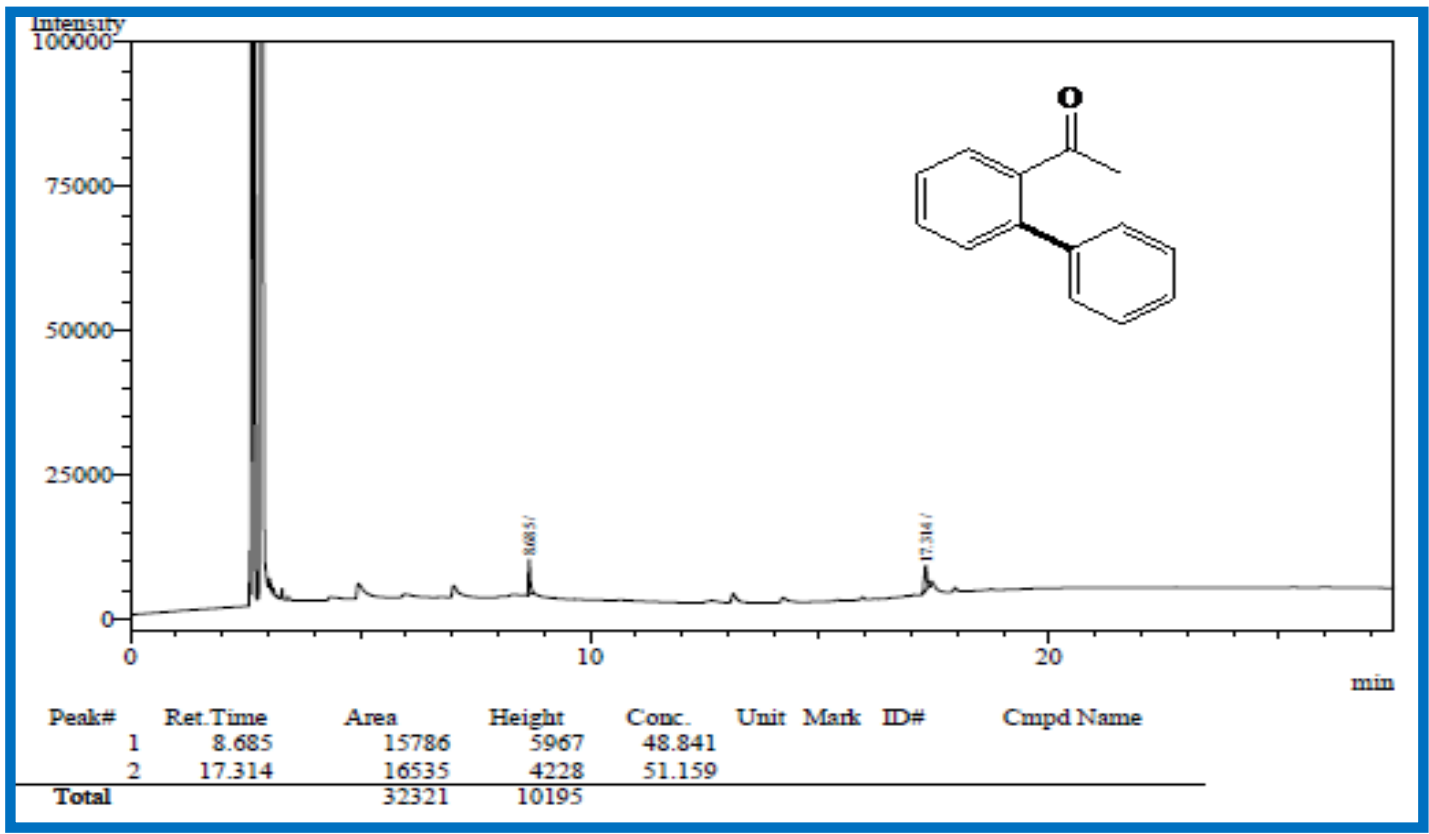

Figure S38. GC of 2-phenylacetophenone

\section{Table 1, Entry 15}

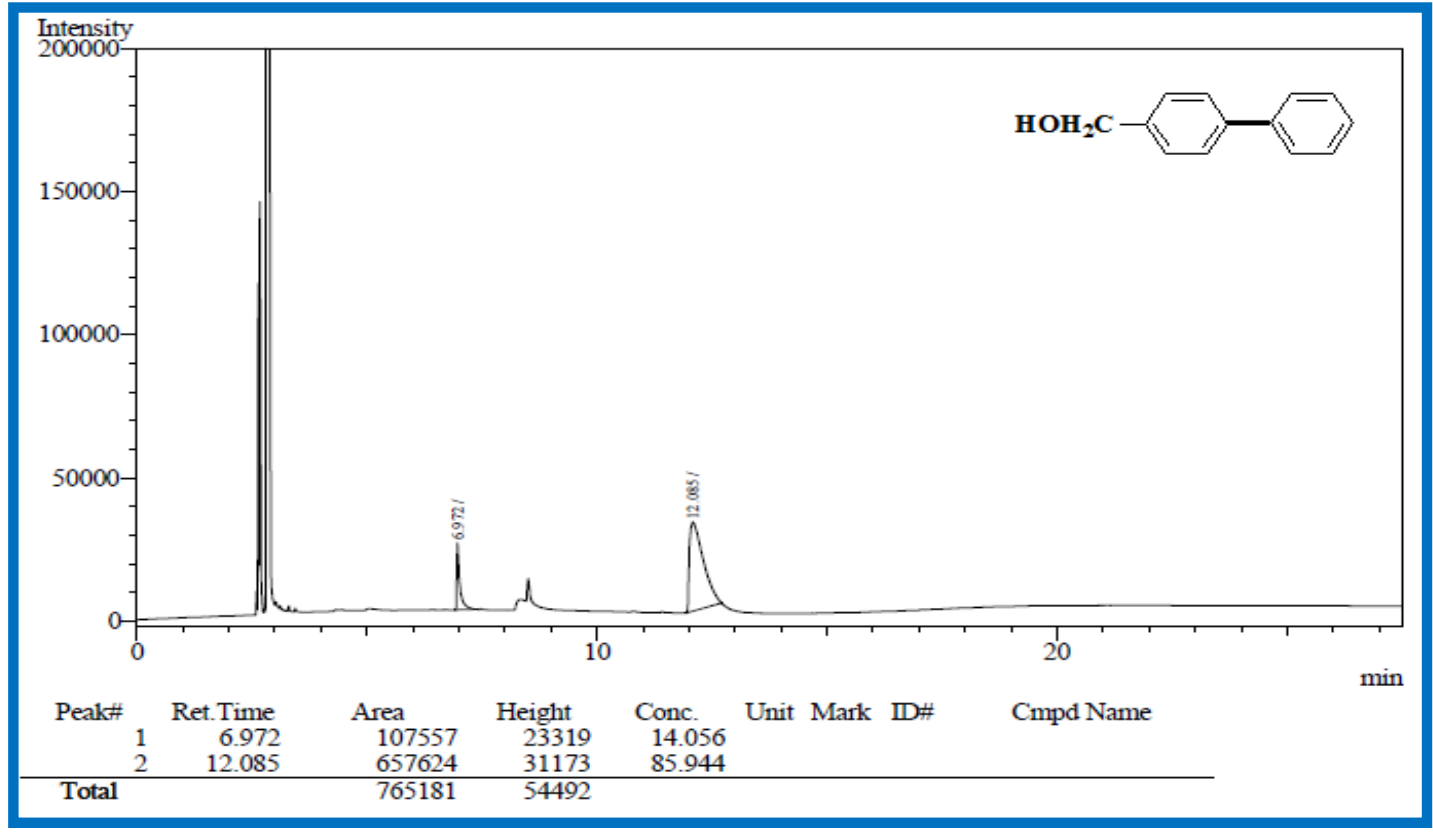

Figure S39. GC of 4-phenylbenzyl alcohol 
5. Table 1 , Entry 16

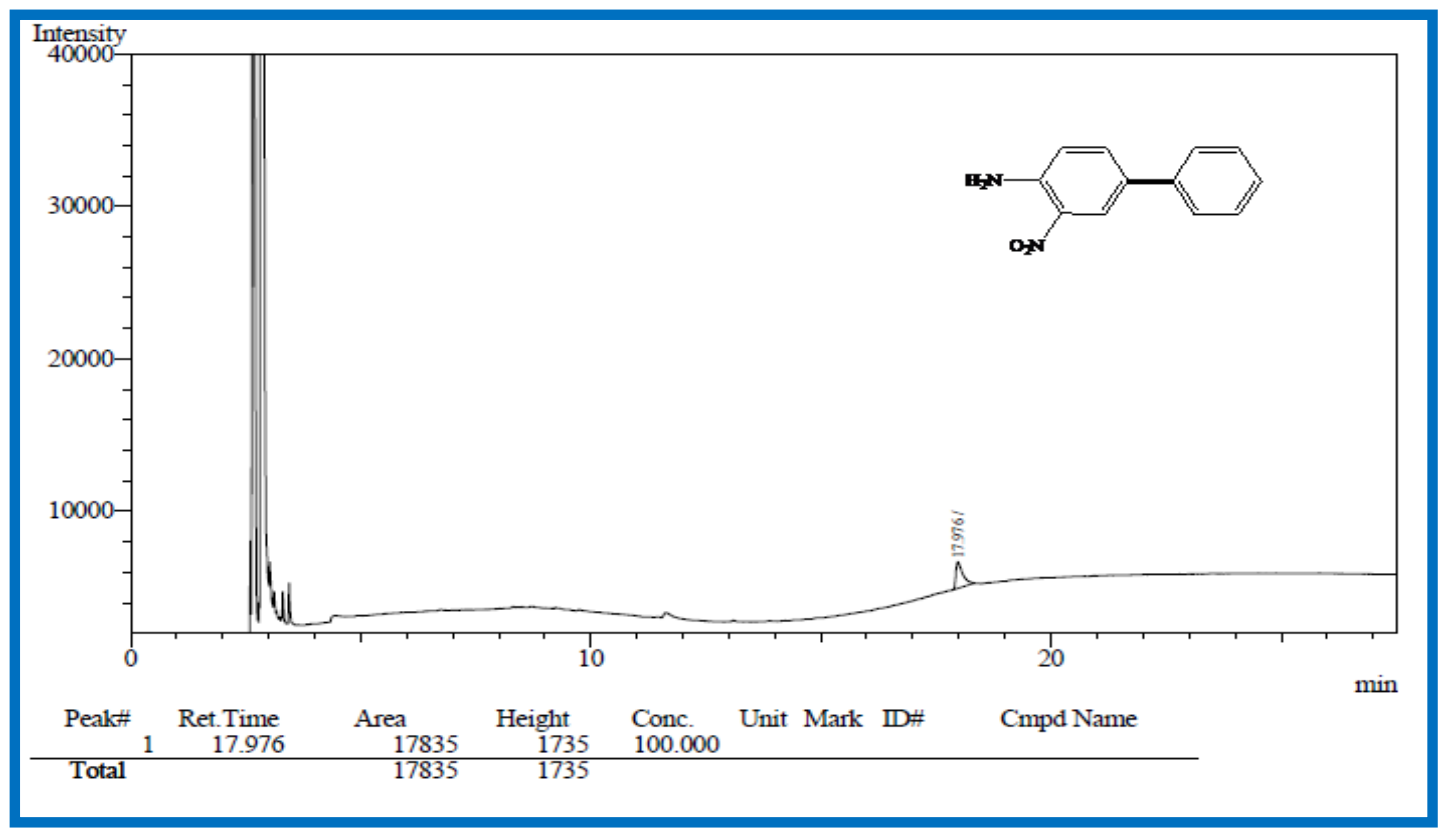

Figure S40. GC of 4-phenyl-2-nitro aniline

6. Table 1, Entry 17

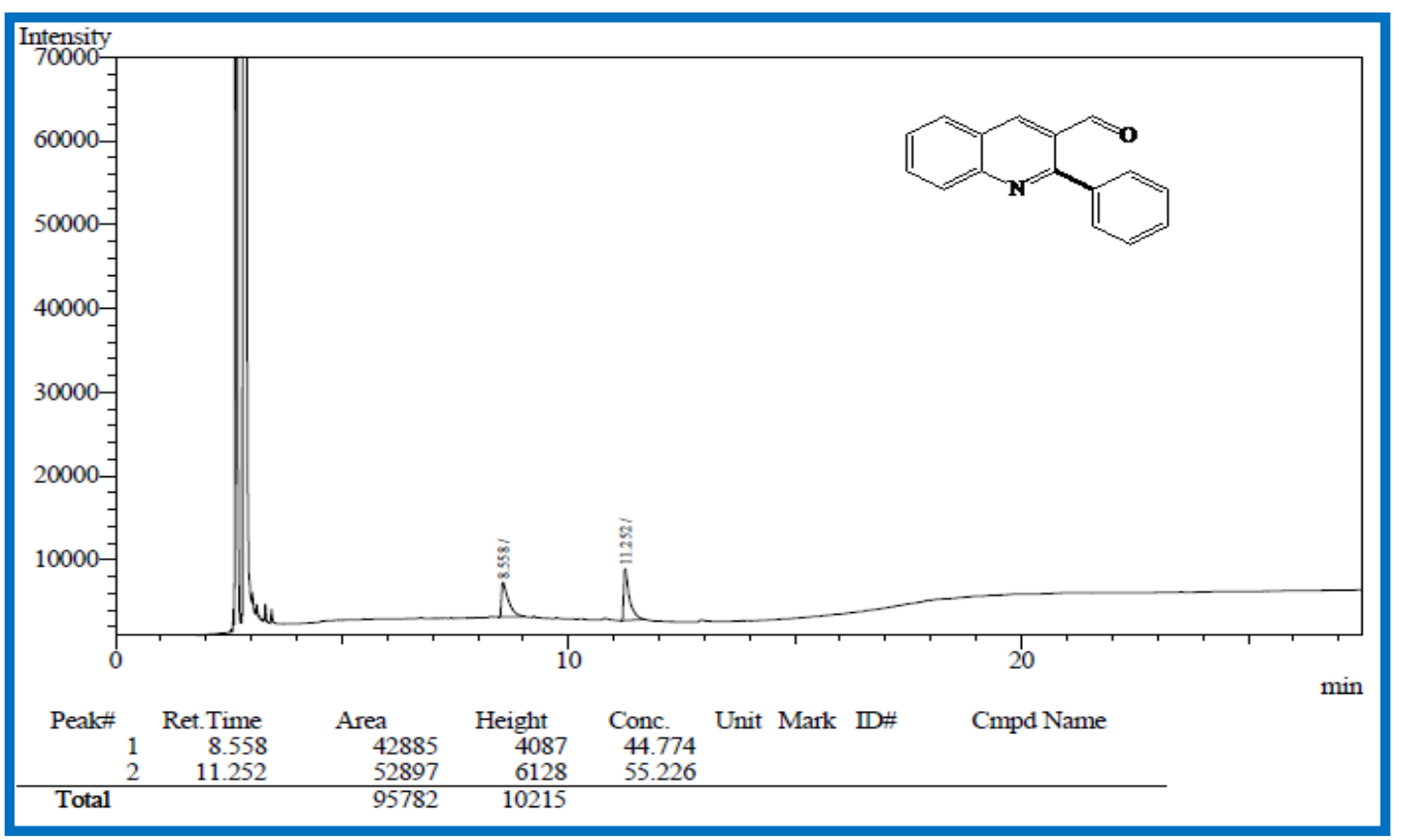

Figure S41. GC of 2-phenyl-3-formylquinoline 
7. Table 1 , Entry 18

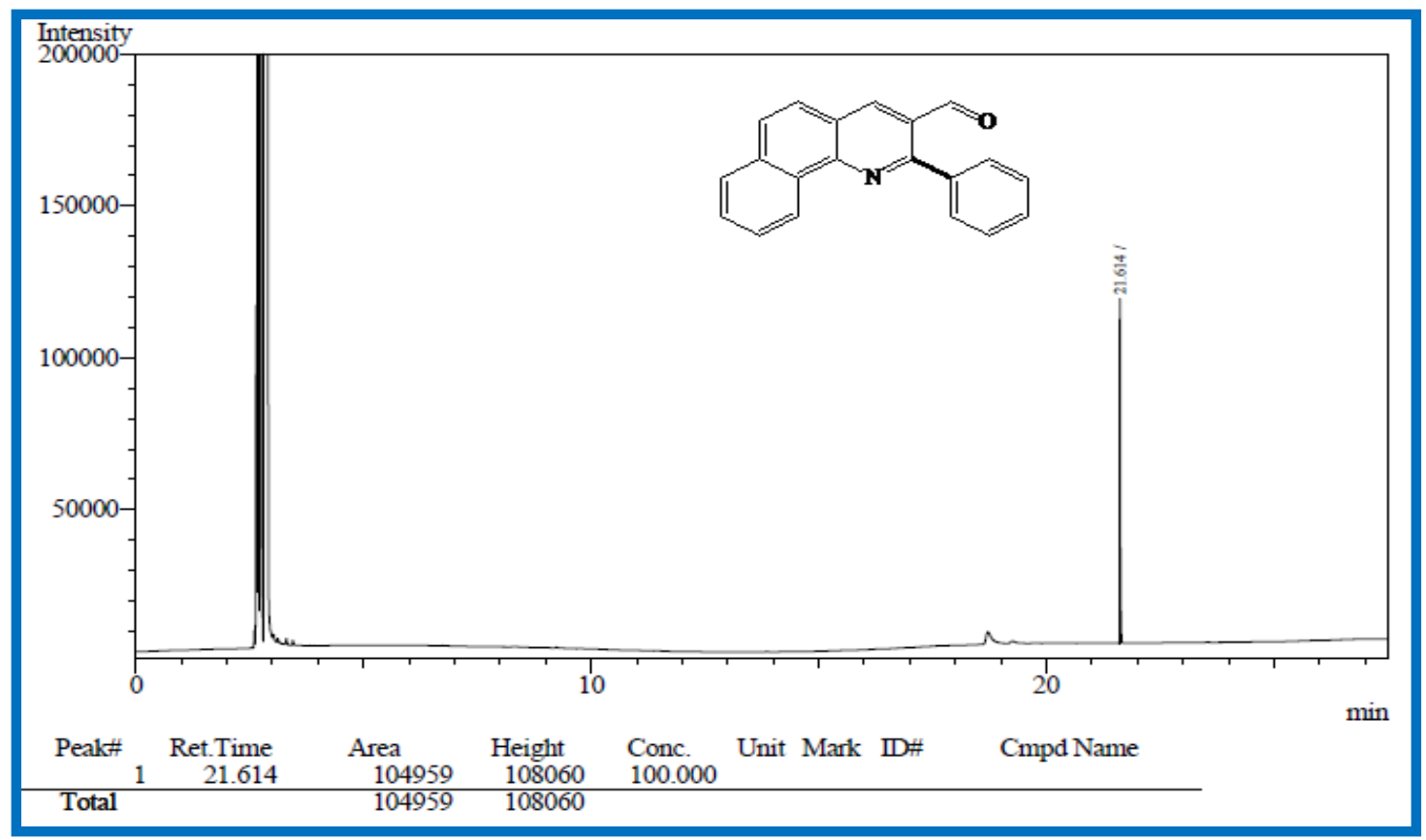

Figure S42. GC of 2-phenyl-3-formylbenzoquinoline

8. Table 1 , Entry 19

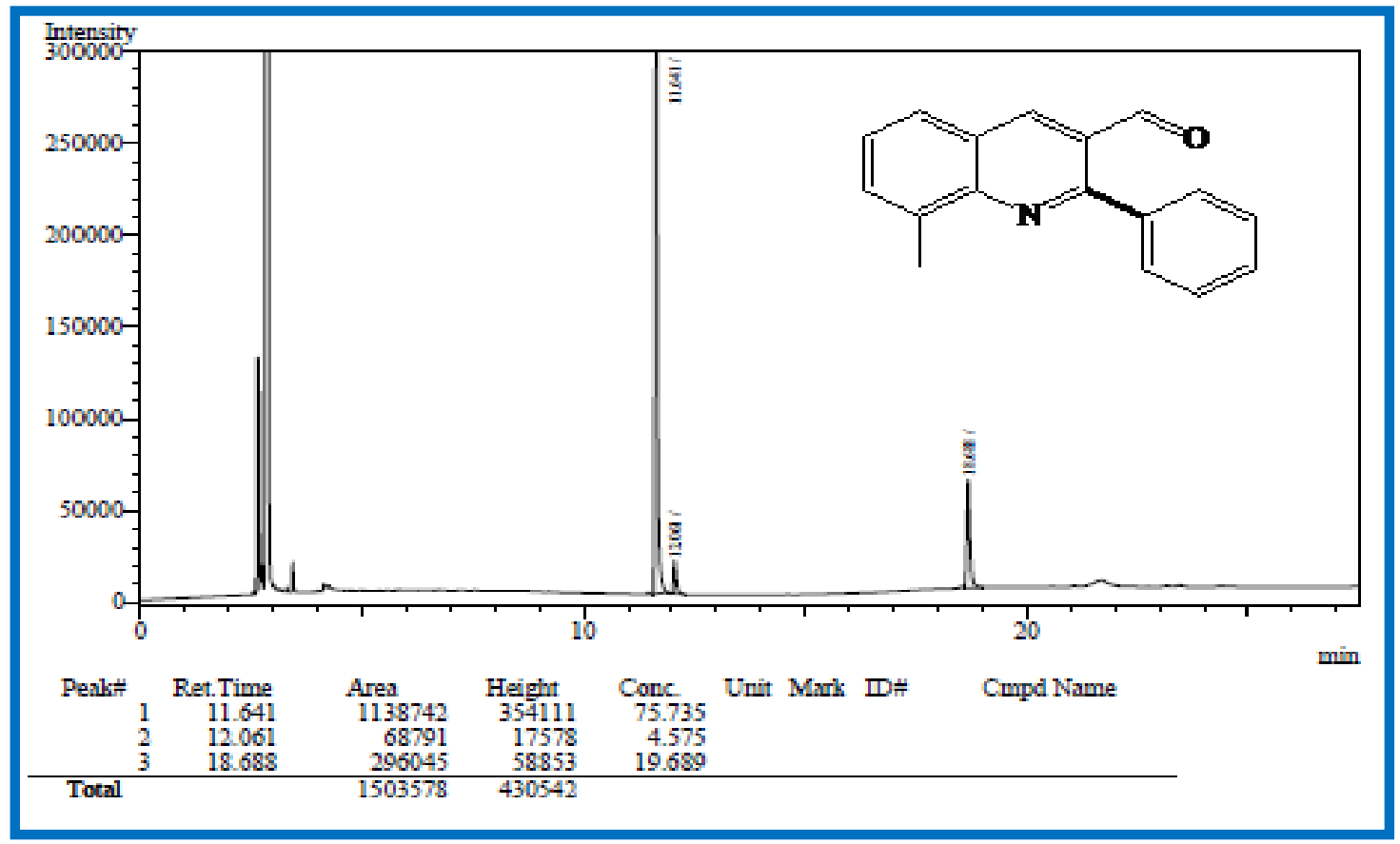

Figure S43. GC of 2-phenyl-3-formyl-8-methylquinoline 


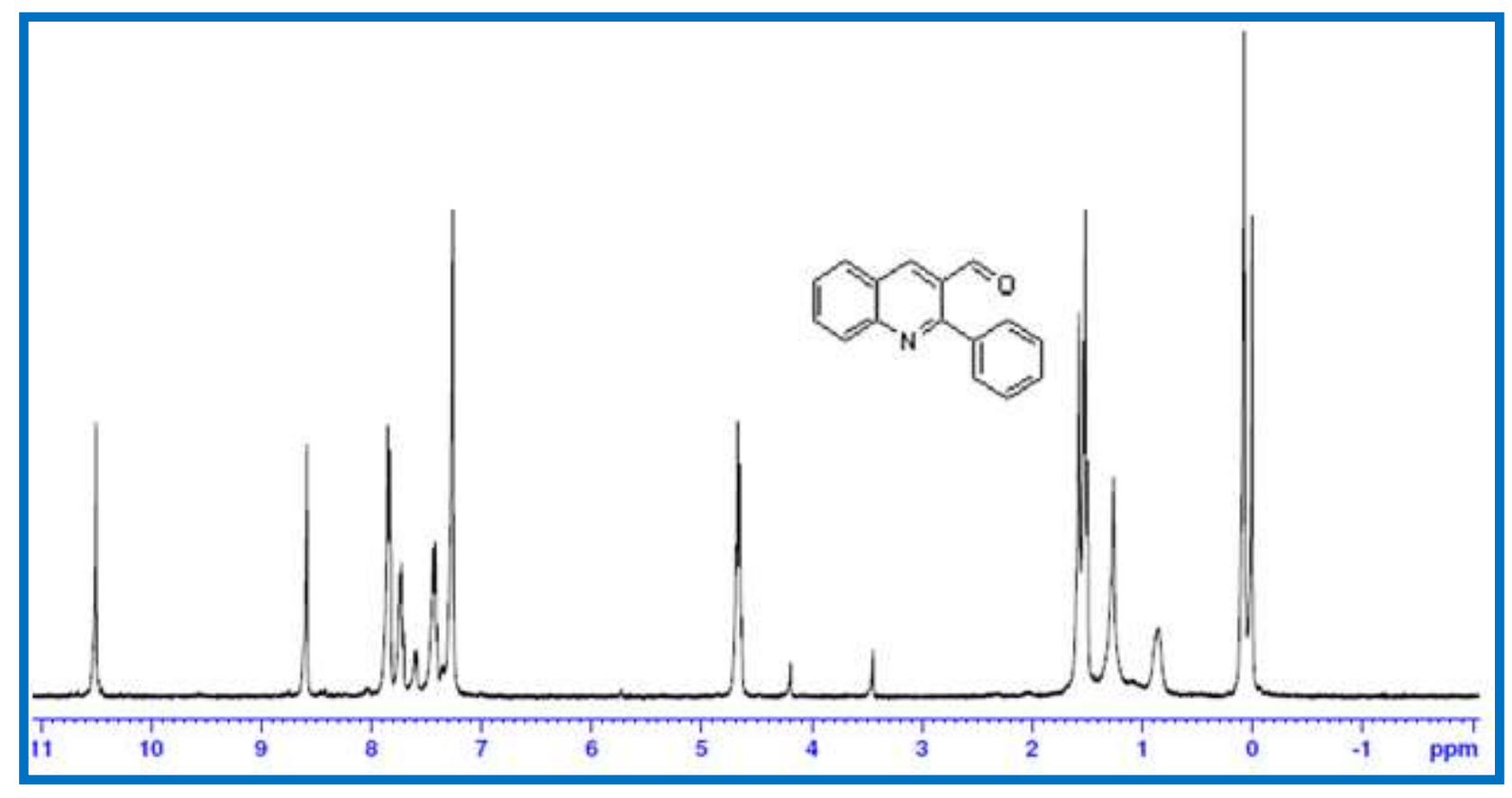

Figure S44. ${ }^{1}$ H NMR spectrum of 2-phenyl-3-formylquinoline

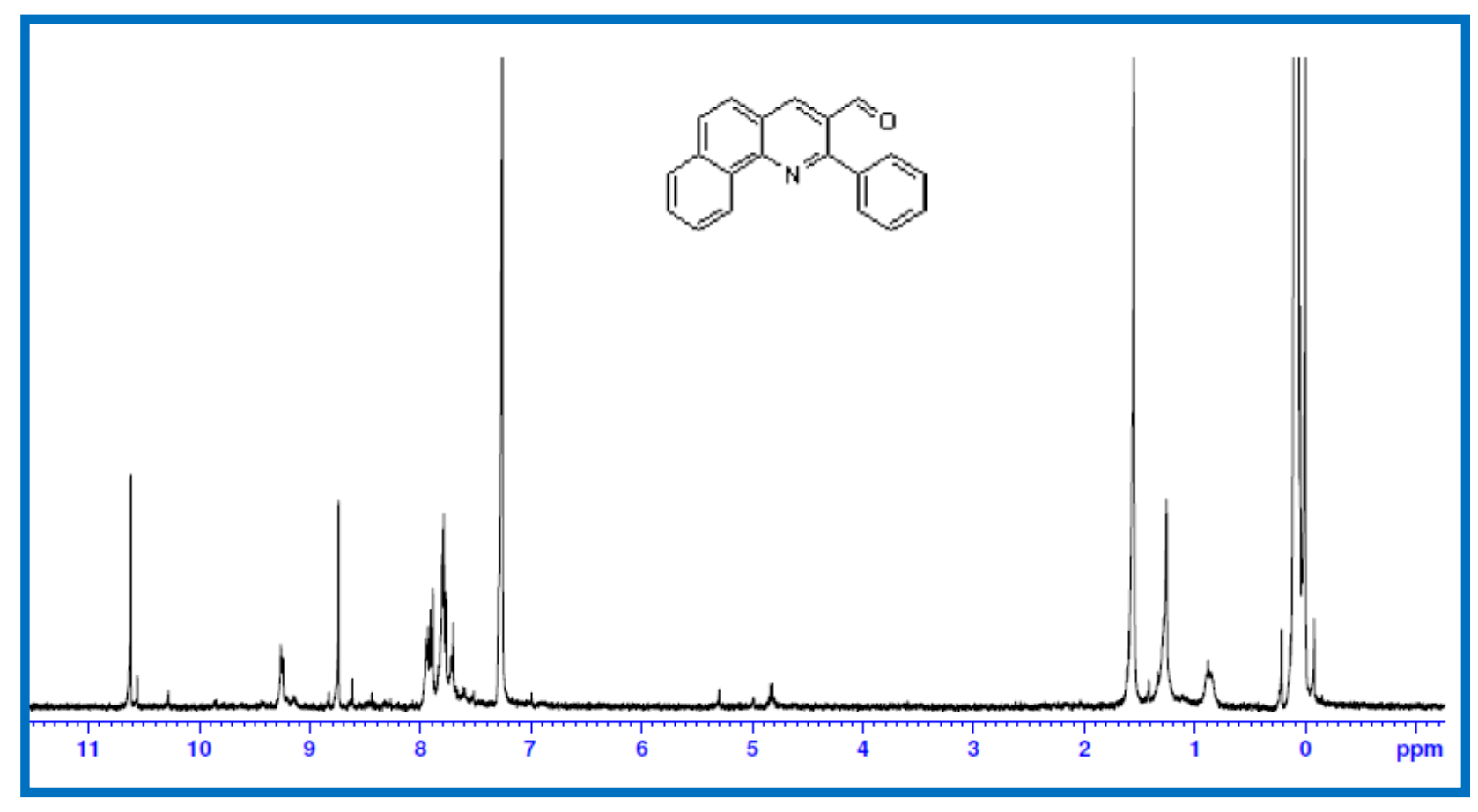

Figure S45. ${ }^{1} \mathrm{H}$ NMR spectrum of 2-phenyl-3-formylbenzoquinoline 


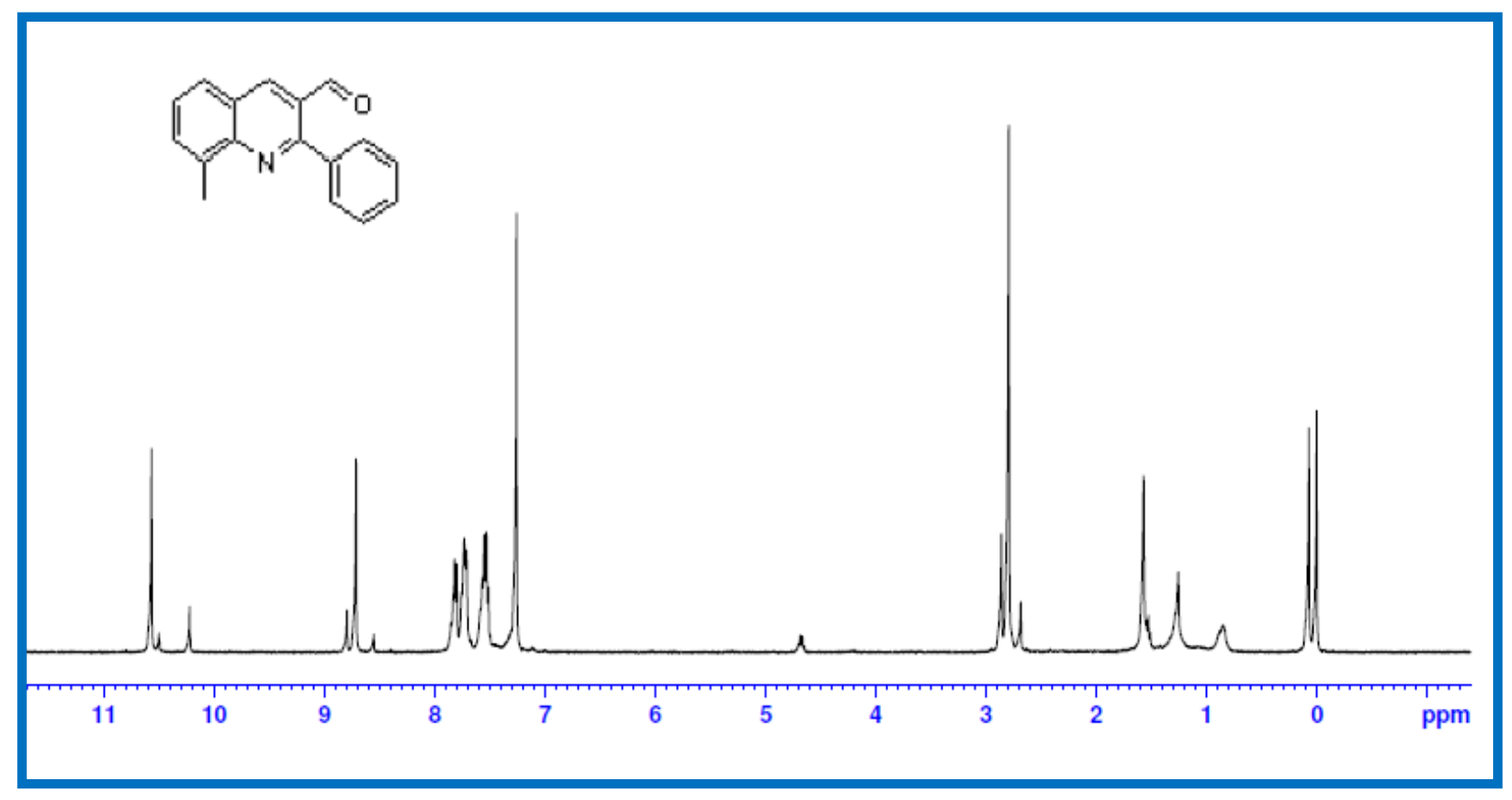

Figure S46. ${ }^{1} \mathrm{H}$ NMR spectrum of 2-phenyl-3-formyl-8-methylquinoline

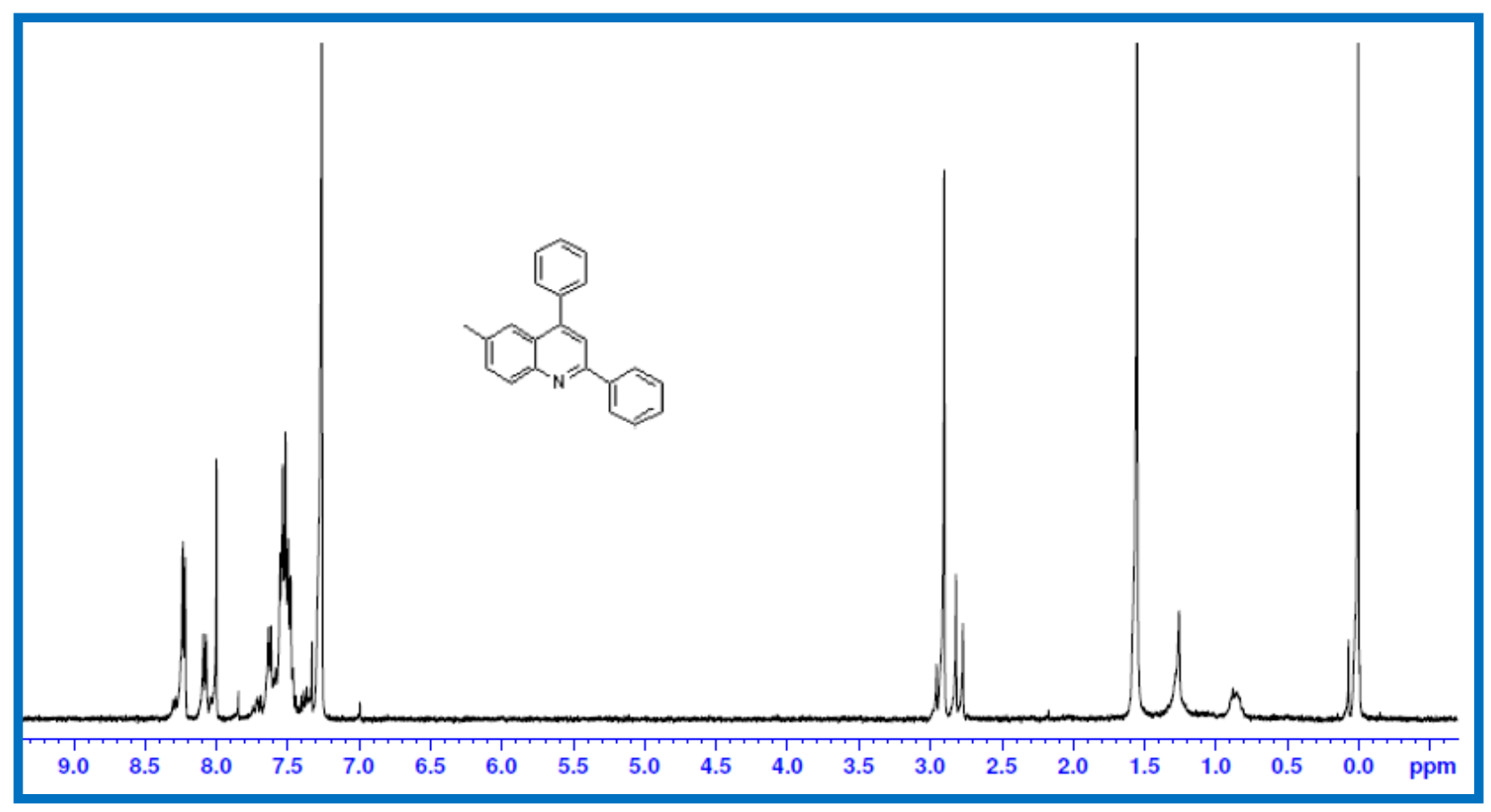

Figure S47. ${ }^{1} \mathrm{H}$ NMR spectrum of 2,4-diphenyl-6-methylquinoline 


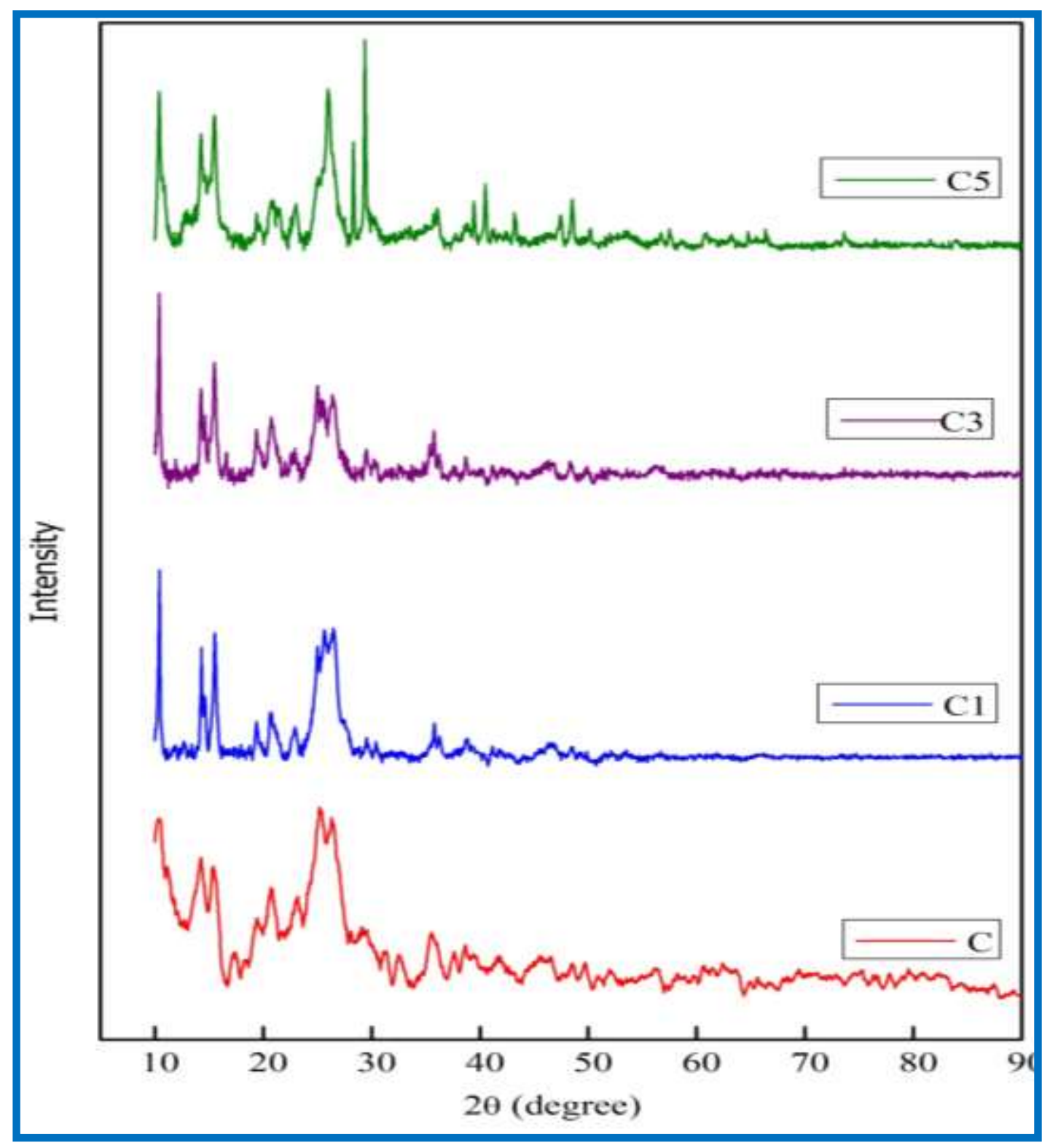

Figure S48. PXRD patterns of the catalysts before usage in catalytic reaction $(\mathbf{C})$ and regenerated after $1^{\text {st }}(\mathbf{C 1}), 3^{\text {rd }}(\mathbf{C 3})$ and $5^{\text {th }}(\mathbf{C 5})$ cycles 


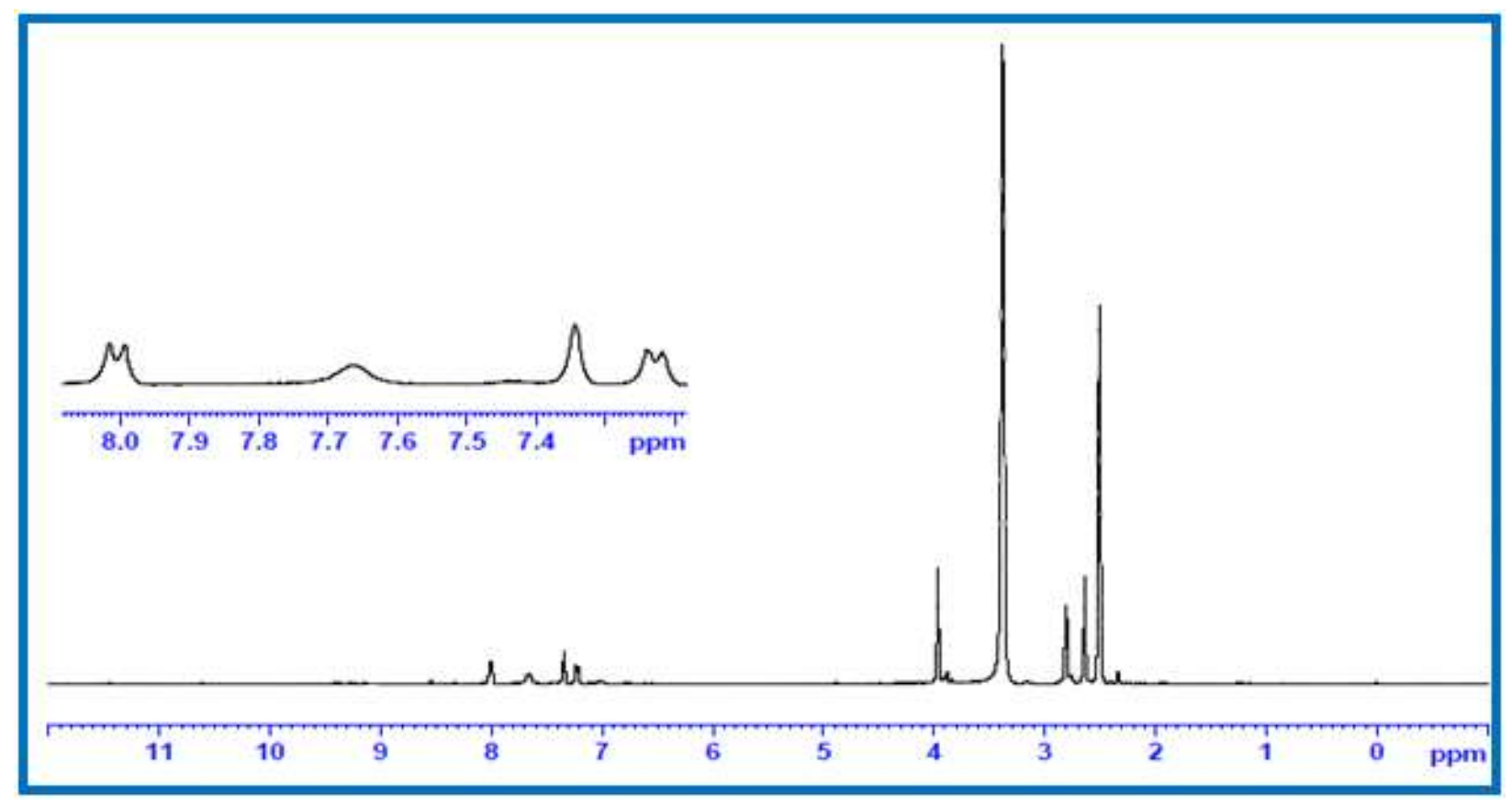

Figure S49. ${ }^{1} \mathrm{H}$ NMR spectrum of the catalyst regenerated after $1^{\text {st }}$ cycle $(\mathbf{C 1})$

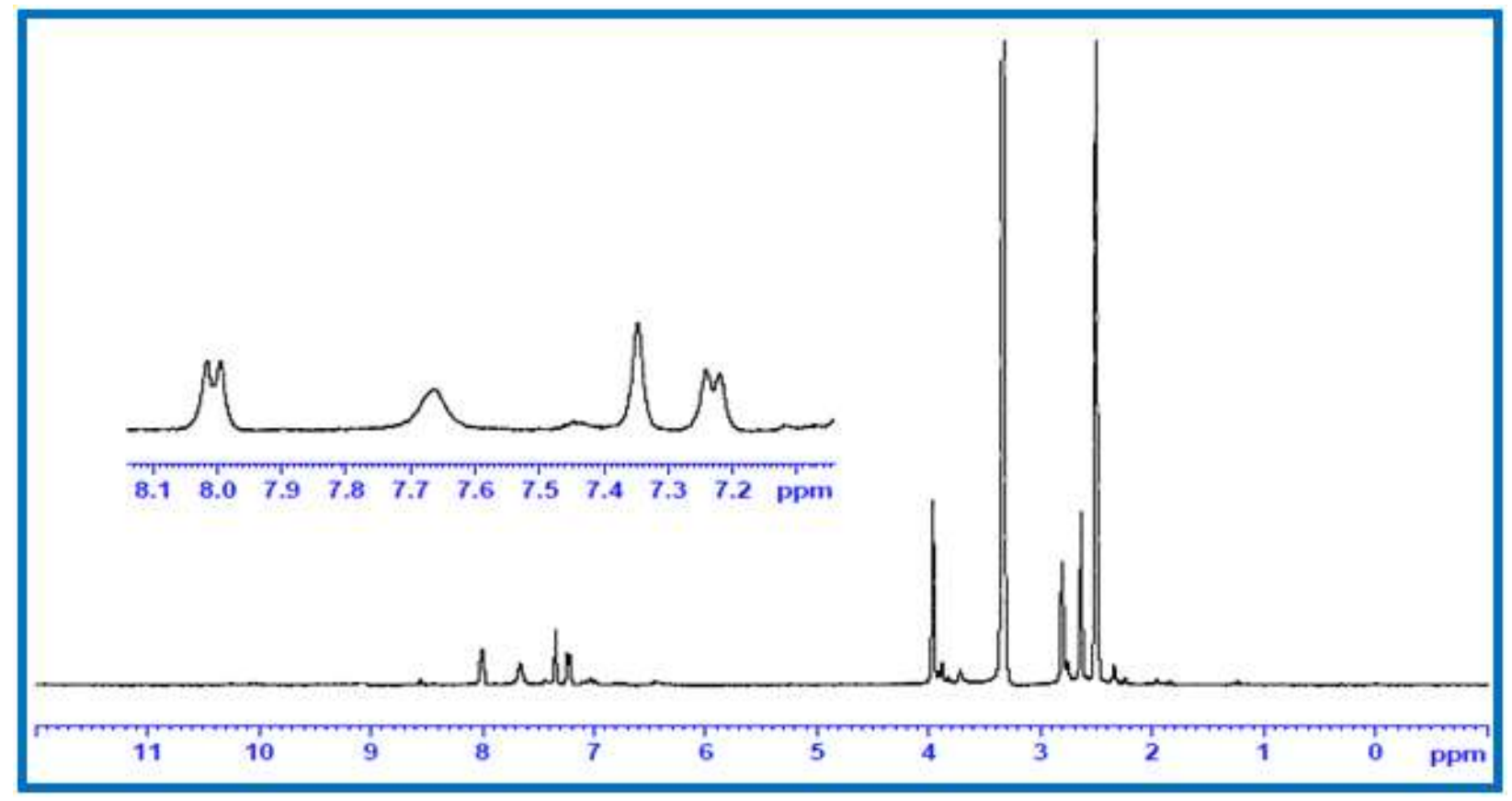

Figure S50. ${ }^{1} \mathrm{H}$ NMR spectrum of the catalyst regenerated after $3^{\text {rd }}$ cycle $(\mathbf{C 3})$ 
Table S1. Electronic spectral data of the complexes 1-4 recorded in dichloromethane at different concentrations $(2 \mu \mathrm{M}, 5 \mu \mathrm{M}$ and $10 \mu \mathrm{M})$ and DMSO $(10 \mu \mathrm{M})$

\begin{tabular}{|c|c|c|c|c|c|c|c|c|}
\hline \multirow[t]{2}{*}{ Complex } & \multicolumn{2}{|r|}{$2 \mu \mathrm{M}$} & \multicolumn{2}{|r|}{$5 \mu \mathrm{M}$} & \multicolumn{2}{|r|}{$10 \mu \mathrm{M}$} & \multicolumn{2}{|c|}{$10 \mu \mathrm{M}(\mathrm{DMSO})$} \\
\hline & $\begin{array}{c}\lambda \\
(\mathbf{n m})\end{array}$ & $\begin{array}{c}\varepsilon \\
\left(\mathbf{d m}^{3} \mathbf{m o l}^{-1} \mathbf{c m}^{-1}\right) \\
\end{array}$ & $\begin{array}{c}\lambda \\
(\mathbf{n m})\end{array}$ & $\begin{array}{c}\varepsilon \\
\left(\mathbf{d m}^{3} \mathrm{~mol}^{-1} \mathbf{c m}^{-1}\right)\end{array}$ & $\begin{array}{c}\lambda \\
(\mathbf{n m})\end{array}$ & $\begin{array}{c}\varepsilon \\
\left(\mathbf{d m}^{3} \mathbf{m o l}^{-1} \mathbf{c m}^{-1}\right)\end{array}$ & $\begin{array}{c}\lambda \\
(\mathbf{n m})\end{array}$ & $\begin{array}{c}\varepsilon \\
\left(\mathbf{d m}^{3} \mathbf{m o l}^{-1} \mathbf{c m}^{-1}\right)\end{array}$ \\
\hline \multirow[t]{3}{*}{1} & 424 & 49967 & 425 & 36935 & 425 & 23510 & 419 & 38496 \\
\hline & 364 & 91276 & 365 & 61574 & 365 & 40639 & 321 & 29840 \\
\hline & 229 & 191779 & 230 & 118451 & 231 & 75902 & 263 & 34910 \\
\hline \multirow[t]{3}{*}{2} & 432 & 47940 & 432 & 34175 & 432 & 25026 & 427 & 89970 \\
\hline & 322 & 26689 & 325 & 18595 & 326 & 13523 & 324 & 36420 \\
\hline & 234 & 255377 & 233 & 121137 & 232 & 70578 & 263 & 33490 \\
\hline \multirow[t]{3}{*}{3} & 434 & 54426 & 433 & 39044 & 434 & 33358 & 428 & 31750 \\
\hline & 326 & 24105 & 327 & 18199 & 328 & 15313 & 327 & 13490 \\
\hline & 234 & 74617 & 232 & 47329 & 232 & 35948 & 266 & 10700 \\
\hline \multirow[t]{4}{*}{4} & 440 & 98782 & 440 & 46709 & 440 & 35708 & 432 & 42400 \\
\hline & 327 & 42803 & 327 & 22191 & 328 & 16727 & 329 & 19540 \\
\hline & 233 & 101549 & 249 & 35730 & 249 & 26718 & 263 & 39850 \\
\hline & & & 229 & 44202 & 231 & 30482 & & \\
\hline
\end{tabular}


Table S2. Crystallographic data of complexes 1-3

\begin{tabular}{|c|c|c|c|}
\hline & Complex 1 & Complex 2 & Complex 3 \\
\hline Empirical formula & $\mathrm{C}_{52} \mathrm{H}_{44} \mathrm{~N}_{12} \mathrm{O}_{12} \mathrm{Pd}_{4} \mathrm{~S}_{4}$ & $\mathrm{C}_{62} \mathrm{H}_{66} \mathrm{~N}_{14} \mathrm{O}_{14} \mathrm{Pd}_{4} \mathrm{~S}_{4}$ & $\mathrm{C}_{60} \mathrm{H}_{60} \mathrm{~N}_{12} \mathrm{O}_{12} \mathrm{Pd}_{4} \mathrm{~S}_{4}$ \\
\hline Formula weight & 1582.83 & 1785.12 & 1695.04 \\
\hline Temperature & $100(2) \mathrm{K}$ & $100(2) \mathrm{K}$ & $100(2) \mathrm{K}$ \\
\hline Wavelength & $0.7107 \mathrm{~A}$ & $0.7107 \AA$ & $1.5418 \AA$ \\
\hline Crystal system & Tetragonal & Monoclinic & Tetragonal \\
\hline Space group & I $4_{1} / \mathrm{a}$ & $\mathrm{C} 2 / \mathrm{c}$ & I $4_{1} / \mathrm{a}$ \\
\hline \multicolumn{4}{|l|}{$\begin{array}{l}\text { Unitcell } \\
\text { dimensions }\end{array}$} \\
\hline $\mathrm{a}$ & 14.414(2)Ă & 16.317(2)A & 14.454(5) Ă \\
\hline b & 14.414(2)Ă & $35.344(4) \mathrm{A}$ & 14.454(5) Ă \\
\hline $\mathrm{c}$ & $36.285(5) \AA$ & 13.404(2)A & 36.108(12) Ă \\
\hline$\alpha$ & $90^{\circ}$ & $90^{\circ}$ & $90^{\circ}$ \\
\hline$\beta$ & $90^{\circ}$ & $123.692(7)$ & $90^{\circ}$ \\
\hline$\gamma$ & $90^{\circ}$ & $90^{\circ}$ & $90^{\circ}$ \\
\hline $\begin{array}{l}\text { Volume } \\
Z\end{array}$ & $\begin{array}{l}7539(2) \check{A}^{3} \\
4\end{array}$ & $\begin{array}{l}6431.7(16) \mathrm{A} 3 \\
4\end{array}$ & $\begin{array}{l}7544.6(6) \check{A}^{3} \\
4\end{array}$ \\
\hline Density & $1.395 \mathrm{Mg} / \mathrm{m}^{3}$ & $1.844 \mathrm{Mg} / \mathrm{m}^{3}$ & $1.492 \mathrm{Mg} / \mathrm{m}^{3}$ \\
\hline $\begin{array}{l}\text { Absorption } \\
\text { coefficient }\end{array}$ & $1.105 \mathrm{~mm}^{-1}$ & $1.309 \mathrm{~mm}^{-1}$ & $9.110 \mathrm{~mm}^{-1}$ \\
\hline$F(000)$ & 3136 & 3584 & 3392 \\
\hline $\begin{array}{l}\theta \text { range for data } \\
\text { collection }\end{array}$ & 3.006 to $27.484^{\circ}$ & 2.289 to $27.511^{\circ}$ & 3.293 to $74.348^{\circ}$ \\
\hline Limiting indices & $\begin{array}{l}-18 \leq h \leq 18 \\
-17 \leq k \leq 18 \\
-47 \leq 1 \leq 47\end{array}$ & $\begin{array}{l}-20 \leq h \leq 21 \\
-45 \leq k \leq 45 \\
-16 \leq l \leq 17\end{array}$ & $\begin{array}{l}-17 \leq h \leq 17 \\
-18 \leq k \leq 17 \\
-44 \leq 1 \leq 44\end{array}$ \\
\hline $\begin{array}{l}\text { Reflections } \\
\text { collected }\end{array}$ & 48087 & 31880 & 17555 \\
\hline $\begin{array}{l}\text { Independent } \\
\text { reflections }\end{array}$ & $\begin{array}{l}4320[\mathrm{R}(\text { int })= \\
0.0941]\end{array}$ & $7371[R(\mathrm{int})=0.0713]$ & $3795[R(\mathrm{int})=0.0382]$ \\
\hline $\begin{array}{l}\text { Absorption } \\
\text { correction }\end{array}$ & Multi-scan & Multi-scan & Multi-scan \\
\hline $\begin{array}{l}\text { Refinement } \\
\text { method }\end{array}$ & $\begin{array}{l}\text { Full-matrix least- } \\
\text { squares on } F^{2}\end{array}$ & $\begin{array}{l}\text { Full-matrix least-squares } \\
\text { on } F^{2}\end{array}$ & $\begin{array}{l}\text { Full-matrix least-squares } \\
\text { on } F^{2}\end{array}$ \\
\hline $\begin{array}{l}\text { Data/restraints/ } \\
\text { parameters }\end{array}$ & $4320 / 126 / 198$ & $7371 / 294 / 458$ & $3795 / 0 / 275$ \\
\hline $\begin{array}{l}\text { Goodness-of-fit on } \\
F^{2}\end{array}$ & 1.052 & 0.993 & 1.023 \\
\hline $\begin{array}{l}\text { Final } R \text { indices } \\
{[I>2 \sigma(I)]}\end{array}$ & $\begin{array}{l}\mathrm{R} 1=0.0904 \\
\mathrm{wR} 2=0.1639\end{array}$ & $\begin{array}{l}\mathrm{R} 1=0.0377 \\
\mathrm{wR} 2=0.0729\end{array}$ & $\begin{array}{l}\mathrm{R} 1=0.0567 \\
\mathrm{wR} 2=0.1403\end{array}$ \\
\hline$R$ indices (all data) & $\begin{array}{l}\mathrm{R} 1=0.1251 \\
\mathrm{wR} 2=0.1790\end{array}$ & $\begin{array}{l}\mathrm{R} 1=0.0673, \\
\mathrm{wR} 2=0.0808\end{array}$ & $\begin{array}{l}\mathrm{R} 1=0.0770 \\
\mathrm{wR} 2=0.1571\end{array}$ \\
\hline
\end{tabular}


Table S3. Selected bond lengths $(\AA)$ and angles $\left({ }^{\circ}\right)$ of complexes1-3

\begin{tabular}{|c|c|c|c|c|c|}
\hline \multicolumn{6}{|l|}{ Bond lengths } \\
\hline \multicolumn{2}{|c|}{ Complex 1} & \multicolumn{2}{|c|}{ Complex 2} & \multicolumn{2}{|c|}{ Complex 3} \\
\hline $\mathrm{Pd}(1)-\mathrm{N}(1)$ & $2.010(6)$ & $\mathrm{Pd}(1)-\mathrm{N}(1)$ & $1.988(3)$ & $\operatorname{Pd}(1)-\mathrm{N}(1)$ & 1.997(6) \\
\hline $\operatorname{Pd}(1)-C(1)$ & $2.060(7)$ & $\mathrm{Pd}(1)-\mathrm{C}(1)$ & $2.043(4)$ & $\mathrm{Pd}(1)-\mathrm{C}(1)$ & $2.065(7)$ \\
\hline $\operatorname{Pd}(1)-S(1)$ & $2.330(19)$ & $\operatorname{Pd}(1)-S(1)$ & $2.347(10)$ & $\operatorname{Pd}(1)-S(1)$ & $2.337(16)$ \\
\hline $\operatorname{Pd}(1)-S(4)$ & $2.336(19)$ & $\operatorname{Pd}(1)-S(2)$ & $2.320(10)$ & $\operatorname{Pd}(1)-S(4)$ & $2.338(18)$ \\
\hline \multirow{4}{*}{$\operatorname{Pd}(1)-\operatorname{Pd}(1)$} & $3.242(13)$ & $\operatorname{Pd}(2)-S(1)$ & $2.351(10)$ & $\operatorname{Pd}(1)-\operatorname{Pd}(1)$ & $3.186(10)$ \\
\hline & & $\operatorname{Pd}(2)-S(2)$ & $2.322(10)$ & & \\
\hline & & $\operatorname{Pd}(1)-\operatorname{Pd}(1)$ & $3.206(7)$ & & \\
\hline & & $\operatorname{Pd}(2)-\mathrm{Pd}(2)$ & $3.195(7)$ & & \\
\hline \multicolumn{6}{|l|}{ Bond angles } \\
\hline \multicolumn{2}{|c|}{ Complex 1} & \multicolumn{2}{|c|}{ Complex 2} & \multicolumn{2}{|c|}{ Complex 3} \\
\hline N1-Pd1-C1 & $81.3(3)$ & N1-Pd1-C1 & 80.99(13) & N1-Pd1-C1 & $80.8(3)$ \\
\hline N1-Pd1-S1 & $82.49(17)$ & N1-Pd1-S1 & $83.25(9)$ & N1-Pd1-S1 & $82.62(17)$ \\
\hline C1-Pd1-S1 & $162.6(2)$ & C1-Pd1-S1 & $160.66(10)$ & C1-Pd1-S1 & $161.5(2)$ \\
\hline N1-Pd1-S1\#1 & $175.32(17)$ & N1-Pd1-S2 & $172.89(9)$ & N1-Pd1-S1\#1 & $174.51(17)$ \\
\hline C1-Pd1-S1\#1 & $103.3(2)$ & $\mathrm{C} 1-\mathrm{Pd} 1-\mathrm{S} 2$ & $103.17(10)$ & C1-Pd1-S1\#1 & $104.5(3)$ \\
\hline S1-Pd1-S1\#1 & 92.99(5) & S2-Pd1-S1 & $93.70(3)$ & S1-Pd1-S1\#1 & $92.28(4)$ \\
\hline N1-Pd1-Pd1\#2 & $88.06(18)$ & N1-Pd1-Pd1\#2 & $84.68(8)$ & N1-Pd1-Pd1\#2 & $88.04(17)$ \\
\hline C1-Pd1-Pd1\#2 & $93.3(2)$ & C1-Pd1-Pd1\#2 & $95.60(10)$ & C1-Pd1-Pd1\#2 & $93.60(19)$ \\
\hline S1-Pd1-Pd1\#2 & $92.54(5)$ & S1-Pd1-Pd1\#2 & $94.03(3)$ & S1-Pd1-Pd1\#2 & $94.07(5)$ \\
\hline S1\#1-Pd1-Pd1\#2 & $90.92(5)$ & S2-Pd1-Pd1\#2 & $89.15(3)$ & S1\#1-Pd1-Pd1\#2 & $90.29(5)$ \\
\hline \multirow{5}{*}{ Pd1-S1-Pd1\#3 } & $115.37(9)$ & $\mathrm{Pd} 1-\mathrm{S} 1-\mathrm{Pd} 2$ & $113.65(4)$ & Pd1-S1-Pd1\#3 & $114.82(8)$ \\
\hline & & Pd1-S2-Pd2 & $116.33(4)$ & & \\
\hline & & $\mathrm{S} 1-\mathrm{Pd} 2-\mathrm{Pd} 2$ & $91.70(3)$ & & \\
\hline & & $\mathrm{S} 2-\mathrm{Pd} 2-\mathrm{Pd} 2$ & $93.29(3)$ & & \\
\hline & & S2-Pd2-S1 & $91.23(4)$ & & \\
\hline
\end{tabular}


Table S4. Hydrogen bonds for complexes 1-3 [A and ${ }^{\circ}$ ]

\begin{tabular}{|c|c|c|c|c|}
\hline $\mathrm{D}-\mathrm{H} \cdots \mathrm{A}$ & $\mathrm{d}(\mathrm{D}-\mathrm{H})$ & $\mathrm{d}(\mathrm{H} \cdots \mathrm{A})$ & $d(D \cdots A)$ & $<(\mathrm{DHA})$ \\
\hline \multicolumn{5}{|l|}{ Complex 1} \\
\hline $\mathrm{N}(3)-\mathrm{H}(1) \mathrm{N}(3) \ldots \mathrm{O}(2)$ & 0.829 & 2.240 & 3.017 & 156.16 \\
\hline \multicolumn{5}{|c|}{$\begin{array}{l}\text { Symmetry operation: }(x, y, z) ;(3 / 4-y, 1 / 4+x, 1 / 4+z) ;(1 / 2-x,-y, 1 / 2+z) ;(3 / 4+y, 3 / 4-x, 3 / 4+z) ; \\
(1 / 2+x, 1 / 2+y, 1 / 2+z) ;(1 / 4-y, 3 / 4+x, 3 / 4+z) ;(-x, 1 / 2-y, z) ;(1 / 4+y, 1 / 4-x, 1 / 4+z) ;(-x,-y,-z) ; \\
(1 / 4+y, 3 / 4-x, 3 / 4-z) ;(1 / 2+x, y, 1 / 2-z) ;(1 / 4-y, 1 / 4+x, 1 / 4-z) ;(1 / 2-x, 1 / 2-y, 1 / 2-z) ;(3 / 4+y, 1 / 4- \\
x, 1 / 4-z) ;(x, 1 / 2+y,-z) ;(3 / 4-y, 3 / 4+x, 3 / 4-z)\end{array}$} \\
\hline \multicolumn{5}{|c|}{ Complex 2} \\
\hline $\mathrm{N}(3)-\mathrm{H} . . . \mathrm{O}(4)$ & 0.802 & 2.116 & 2.882 & 159.70 \\
\hline $\mathrm{N}(6)-\mathrm{H} . . . \mathrm{O}(1)$ & 0.800 & 2.209 & 2.906 & 145.94 \\
\hline \multicolumn{5}{|c|}{$\begin{array}{l}\text { Symmetry operation: }(x, y, z) ;(-x, y, 1 / 2-z) ;(1 / 2+x, 1 / 2+y, z) ;(1 / 2-x, 1 / 2+y, 1 / 2-z) ;(-x,-y,-z) \\
(x,-y, 1 / 2+z) ;(1 / 2-x, 1 / 2-y,-z) ;(1 / 2+x, 1 / 2-y, 1 / 2+z)\end{array}$} \\
\hline \multicolumn{5}{|l|}{ Complex 3} \\
\hline $\mathrm{N}(3)-\mathrm{H}(1) \mathrm{N}(3) \ldots \mathrm{O}(2)$ & 0.892 & 2.142 & 2.971 & 154.18 \\
\hline \multicolumn{5}{|c|}{$\begin{array}{l}\text { Symmetry operation: }(x, y, z) ;(1 / 2-x,-y, 1 / 2+z) ;(3 / 4-y, 1 / 4+x, 1 / 4+z) ;(3 / 4+y, 3 / 4-x, 3 / 4+z) ; \\
(1 / 2+x, 1 / 2+y, 1 / 2+z) ;(-x, 1 / 2-y, z) ;(1 / 4-y, 3 / 4+x, 3 / 4+z) ;(1 / 4+y, 1 / 4-x, 1 / 4+z) ;(-x,-y,-z) ; \\
(1 / 2+x, y, 1 / 2-z) ;(1 / 4+y, 3 / 4-x, 3 / 4-z) ;(1 / 4-y, 1 / 4+x, 1 / 4-z) ;(1 / 2-x, 1 / 2-y, 1 / 2-z) ;(x, 1 / 2+y,-z) ; \\
(3 / 4+y, 1 / 4-x, 1 / 4-z) ;(3 / 4-y, 3 / 4+x, 3 / 4-z)\end{array}$} \\
\hline
\end{tabular}


Table S5. Experimental and theoretical bond lengths of complexes 1-4

\begin{tabular}{|c|c|c|c|}
\hline Complex & & Experimental & DFT \\
\hline \multirow[t]{4}{*}{1} & $\operatorname{Pd}(1)-\mathrm{N}(1)$ & 2.010 & 2.033 \\
\hline & $\operatorname{Pd}(1)-C(1)$ & 2.060 & 2.065 \\
\hline & $\operatorname{Pd}(1)-S(1)$ & 2.330 & 2.496 \\
\hline & $\operatorname{Pd}(1)-S(4)$ & 2.336 & 2.505 \\
\hline \multirow[t]{6}{*}{2} & $\mathrm{Pd}(1)-\mathrm{N}(1)$ & 1.988 & 2.036 \\
\hline & $\operatorname{Pd}(1)-C(1)$ & 2.043 & 2.043 \\
\hline & $\operatorname{Pd}(1)-S(1)$ & 2.347 & 2.512 \\
\hline & $\operatorname{Pd}(1)-S(2)$ & 2.320 & 2.491 \\
\hline & $\operatorname{Pd}(2)-S(1)$ & 2.351 & 2.499 \\
\hline & $\operatorname{Pd}(2)-S(2)$ & 2.322 & 2.475 \\
\hline \multirow[t]{4}{*}{3} & $\mathrm{Pd}(1)-\mathrm{N}(1)$ & 1.997 & 2.034 \\
\hline & $\operatorname{Pd}(1)-C(1)$ & 2.065 & 2.066 \\
\hline & $\operatorname{Pd}(1)-S(1)$ & 2.337 & 2.503 \\
\hline & $\operatorname{Pd}(1)-S(4)$ & 2.338 & 2.491 \\
\hline \multirow[t]{4}{*}{4} & Pd-N & - & 2.064 \\
\hline & $\mathrm{Pd}-\mathrm{O}$ & - & 2.086 \\
\hline & Pd-S & - & 2.335 \\
\hline & $\mathrm{Pd}-\mathrm{Cl}$ & - & 2.368 \\
\hline
\end{tabular}

Table S6. Calculated band gap for the complexes 1-4

\begin{tabular}{|c|c|c|c|c|c|c|c|c|c|}
\hline \multirow{2}{*}{ Complex } & \multicolumn{2}{|c|}{ Without solvent } & \multicolumn{3}{c|}{ DMSO } & \multicolumn{3}{c|}{ Ethanol+water } \\
\cline { 2 - 10 } & $\begin{array}{c}\text { HOMO } \\
(\mathrm{eV})\end{array}$ & $\begin{array}{c}\text { LUMO } \\
(\mathrm{eV})\end{array}$ & $\begin{array}{c}\text { Band } \\
\text { gap } \\
(\mathrm{eV})\end{array}$ & $\begin{array}{c}\text { HOMO } \\
(\mathrm{eV})\end{array}$ & $\begin{array}{c}\text { LUMO } \\
(\mathrm{eV})\end{array}$ & $\begin{array}{c}\text { Band } \\
\text { gap } \\
(\mathrm{eV})\end{array}$ & $\begin{array}{c}\text { HOMO } \\
(\mathrm{eV})\end{array}$ & $\begin{array}{c}\text { LUMO } \\
(\mathrm{eV})\end{array}$ & $\begin{array}{c}\text { Band } \\
\text { gap } \\
(\mathrm{eV})\end{array}$ \\
\hline $\mathbf{1}$ & -5.70 & -2.71 & 2.99 & -5.74 & -2.73 & 3.01 & -5.74 & -2.72 & 3.02 \\
\hline $\mathbf{2}$ & -5.66 & -2.68 & 2.98 & -5.75 & -2.73 & 3.02 & -5.75 & -2.73 & 3.02 \\
\hline $\mathbf{3}$ & -5.56 & -2.57 & 2.99 & -5.74 & -2.73 & 3.01 & -5.74 & -2.72 & 3.02 \\
\hline $\mathbf{4}$ & -5.74 & -3.28 & 2.46 & -5.89 & -3.11 & 2.78 & -5.89 & -3.11 & 2.78 \\
\hline
\end{tabular}


Table S7. Percentage composition of frontier molecular orbitals of the complexes

\begin{tabular}{|c|c|c|c|c|c|c|c|c|c|c|c|c|c|}
\hline \multirow{2}{*}{ Complex } & \multirow{2}{*}{$\begin{array}{l}\text { MO- } \\
\text { Level }\end{array}$} & \multirow{2}{*}{ Occupancy } & \multicolumn{3}{|c|}{ Pd } & \multicolumn{2}{|c|}{ C } & \multicolumn{2}{|c|}{$\mathbf{N}$} & \multicolumn{2}{|c|}{ S } & \multicolumn{2}{|c|}{ O } \\
\hline & & & $\mathbf{S}$ & $\mathbf{p}$ & d & $\mathbf{s}$ & $\mathbf{p}$ & $\mathbf{S}$ & p & $\mathbf{S}$ & $\mathbf{p}$ & $\mathbf{s}$ & p \\
\hline \multirow{12}{*}{1} & $\mathrm{~L}^{+5}$ & 1.89 & 0 & 0 & 0 & 0 & 0 & 0 & 0 & 46.8 & 53.2 & 0 & 0 \\
\hline & $\mathrm{L}^{+4}$ & 1.83 & 0 & 0 & 0 & 0 & 0 & 0 & 0 & 0 & 0 & 0.3 & 99.7 \\
\hline & $\mathrm{L}^{+3}$ & 1.97 & 0 & 0 & 0 & 0 & 0 & 0 & 0 & 0 & 0 & 41.5 & 58.5 \\
\hline & $\mathrm{L}^{+2}$ & 1.84 & 0 & 0 & 0 & 0 & 0 & 0 & 0 & 0 & 0 & 0.04 & 99.96 \\
\hline & $\mathrm{L}^{+1}$ & 1.97 & 0 & 0 & 0 & 0 & 0 & 0 & 0 & 0 & 0 & 63.3 & 36.7 \\
\hline & $\mathrm{L}$ & 1.74 & 0 & 0 & 0 & 0 & 0 & 0 & 0 & 0 & 0 & 0.01 & 99.99 \\
\hline & $\mathrm{H}$ & 1.96 & 0 & 0 & 0 & 0 & 0 & 0 & 0 & 0 & 0 & 36.7 & 63.3 \\
\hline & $\mathrm{H}^{-1}$ & 1.74 & 0 & 0 & 0 & 0 & 0 & 0.1 & 99.9 & 0 & 0 & 0 & 0 \\
\hline & $\mathrm{H}^{-2}$ & 1.85 & 0 & 0 & 0 & 0 & 0 & 36.5 & 63.5 & 0 & 0 & 0 & 0 \\
\hline & $\mathrm{H}^{-3}$ & 1.61 & 0 & 0 & 0 & 0 & 0 & 33.7 & 66.3 & 0 & 0 & 0 & 0 \\
\hline & $\mathrm{H}^{-4}$ & 0.05 & 0.04 & 99.9 & 0.1 & 0 & 0 & 0 & 0 & 0 & 0 & 0 & 0 \\
\hline & $\mathrm{H}^{-5}$ & 0.12 & 0.2 & 99.2 & 0.6 & 0 & 0 & 0 & 0 & 0 & 0 & 0 & 0 \\
\hline \multirow{12}{*}{2} & $\mathrm{~L}^{+5}$ & 0.06 & 0.1 & 99.8 & 0.08 & 0 & 0 & 0 & 0 & 0 & 0 & 0 & 0 \\
\hline & $\mathrm{L}^{+4}$ & 0.11 & 0.11 & 99.1 & 0.74 & 0 & 0 & 0 & 0 & 0 & 0 & 0 & 0 \\
\hline & $\mathrm{L}^{+3}$ & 0.12 & 0.9 & 97.8 & 1.32 & 0 & 0 & 0 & 0 & 0 & 0 & 0 & 0 \\
\hline & $\mathrm{L}^{+2}$ & 0.4 & 88.2 & 0.42 & 11.4 & 0 & 0 & 0 & 0 & 0 & 0 & 0 & 0 \\
\hline & $\mathrm{L}^{+1}$ & 1.94 & 0.1 & 0 & 99.9 & 0 & 0 & 0 & 0 & 0 & 0 & 0 & 0 \\
\hline & $\mathrm{L}$ & 1.97 & 0.21 & 0.01 & 99.8 & 0 & 0 & 0 & 0 & 0 & 0 & 0 & 0 \\
\hline & $\mathrm{H}$ & 1.98 & 0.6 & 0.05 & 99.3 & 0 & 0 & 0 & 0 & 0 & 0 & 0 & 0 \\
\hline & $\mathrm{H}^{-1}$ & 1.99 & 1.32 & 0.05 & 98.6 & 0 & 0 & 0 & 0 & 0 & 0 & 0 & 0 \\
\hline & $\mathrm{H}^{-2}$ & 0.06 & 0 & 99.9 & 0.06 & 0 & 0 & 0 & 0 & 0 & 0 & 0 & 0 \\
\hline & $\mathrm{H}^{-3}$ & 0.12 & 0.38 & 98.6 & 0.9 & 0 & 0 & 0 & 0 & 0 & 0 & 0 & 0 \\
\hline & $\mathrm{H}^{-4}$ & 0.12 & 0.6 & 98.2 & 1.2 & 0 & 0 & 0 & 0 & 0 & 0 & 0 & 0 \\
\hline & $\mathrm{H}^{-5}$ & 0.38 & 89.6 & 0.4 & 10.1 & 0 & 0 & 0 & 0 & 0 & 0 & 0 & 0 \\
\hline \multirow{12}{*}{3} & $\mathrm{~L}^{+5}$ & 1.68 & 1.12 & 0.03 & 98.85 & 0 & 0 & 0 & 0 & 0 & 0 & 0 & 0 \\
\hline & $\mathrm{L}^{+4}$ & 1.84 & 0 & 0 & 0 & 0 & 0 & 0 & 0 & 0 & 0 & 0.4 & 99.96 \\
\hline & $\mathrm{L}^{+3}$ & 1.97 & 0 & 0 & 0 & 0 & 0 & 0 & 0 & 0 & 0 & 66.3 & 36.7 \\
\hline & $\mathrm{L}^{+2}$ & 1.74 & 0 & 0 & 0 & 0 & 0 & 0 & 0 & 0 & 0 & 0.01 & 99.99 \\
\hline & $\mathrm{L}^{+1}$ & 1.96 & 0 & 0 & 0 & 0 & 0 & 0 & 0 & 0 & 0 & 36.7 & 63.3 \\
\hline & $\mathrm{L}$ & 1.69 & 0 & 0 & 0 & 0 & 0 & 0.08 & 99.92 & 0 & 0 & 0 & 0 \\
\hline & $\mathrm{H}$ & 1.85 & 0 & 0 & 0 & 0 & 0 & 36.4 & 63.6 & 0 & 0 & 0 & 0 \\
\hline & $\mathrm{H}^{-1}$ & 1.62 & 0 & 0 & 0 & 0 & 0 & 33.5 & 66.5 & 0 & 0 & 0 & 0 \\
\hline & $\mathrm{H}^{-2}$ & 1.83 & 0 & 0 & 0 & 0 & 0 & 0 & 0 & 0 & 0 & 0.3 & 99.7 \\
\hline & $\mathrm{H}^{-3}$ & 1.97 & 0 & 0 & 0 & 0 & 0 & 0 & 0 & 0 & 0 & 41.6 & 58.4 \\
\hline & $\mathrm{H}^{-4}$ & 1.61 & 0 & 0 & 0 & 0 & 0 & 0 & 0 & 7.9 & 92.1 & 0 & 0 \\
\hline & $\mathrm{H}^{-5}$ & 1.68 & 0 & 0 & 0 & 0 & 0 & 0 & 0 & 33.7 & 66.3 & 0 & 0 \\
\hline \multirow{12}{*}{4} & $\mathrm{~L}^{+5}$ & 0.01 & 0 & 0 & 0 & 0.1 & 99.9 & 0 & 0 & 0 & 0 & 0 & 0 \\
\hline & $\mathrm{L}^{+4}$ & 1.74 & 0 & 0 & 0 & 0 & 0 & 0 & 0 & 0.05 & 99.95 & 0 & 0 \\
\hline & $\mathrm{L}^{+3}$ & 1.95 & 0 & 0 & 0 & 0 & 0 & 0 & 0 & 73.9 & 26.20 & 0 & 0 \\
\hline & $\mathrm{L}^{+2}$ & 1.95 & 0 & 0 & 0 & 0 & 100 & 0 & 0 & 0 & 0 & 0 & 0 \\
\hline & $\mathrm{L}^{+1}$ & 9.96 & 0 & 0 & 0 & 61.4 & 38.6 & 0 & 0 & 0 & 0 & 0 & 0 \\
\hline & $\mathrm{L}$ & 1.98 & 0 & 0 & 0 & 28.1 & 71.9 & 0 & 0 & 0 & 0 & 0 & 0 \\
\hline & $\mathrm{H}$ & 1.96 & 0 & 0 & 0 & 28.06 & 71.94 & 0 & 0 & 0 & 0 & 0 & 0 \\
\hline & $\mathrm{H}^{-1}$ & 0.08 & 0 & 99.86 & 0.14 & 0 & 0 & 0 & 0 & 0 & 0 & 0 & 0 \\
\hline & $\mathrm{H}^{-2}$ & 0.15 & 8.73 & 87.92 & 3.35 & 0 & 0 & 0 & 0 & 0 & 0 & 0 & 0 \\
\hline & $\mathrm{H}^{-3}$ & 0.17 & 13.5 & 83.4 & 3.1 & 0 & 0 & 0 & 0 & 0 & 0 & 0 & 0 \\
\hline & $\mathrm{H}^{-4}$ & 1.95 & 0 & 0.04 & 99.96 & 0 & 0 & 0 & 0 & 0 & 0 & 0 & 0 \\
\hline & $\mathrm{H}^{-5}$ & 1.98 & 0 & 0.1 & 99.8 & 0 & 0 & 0 & 0 & 0 & 0 & 0 & 0 \\
\hline
\end{tabular}


Table S8. Details regarding the wavelength, orbitals involved and energy of transition of the complexes 1-4

\begin{tabular}{ccccccc}
\hline Complex & $\begin{array}{c}\text { Wavelength } \\
(\mathbf{n m})\end{array}$ & $\begin{array}{c}\text { Oscillator } \\
\text { strength }\end{array}$ & $\begin{array}{c}\text { Energy } \\
(\mathbf{e V})\end{array}$ & Transition & Orbital & $\begin{array}{c}\text { Respective } \\
\text { energy of } \\
\text { transition } \\
(\mathbf{e V})\end{array}$ \\
\hline $\mathbf{1}$ & 322.1 & 0.0347 & 3.848 & $297-317$ & $\mathrm{H}^{-19}-\mathrm{L}$ & 0.402 \\
& 419.2 & 0.0799 & 2.957 & $313-320$ & $\mathrm{H}^{-3}-\mathrm{L}^{3}$ & 0.356 \\
& 481.1 & 0.0562 & 2.577 & $314-317$ & $\mathrm{H}^{2}-\mathrm{L}$ & 0.511 \\
\hline $\mathbf{2}$ & 324.6 & 0.0055 & 3.818 & $332-354$ & $\mathrm{H}^{-20}-\mathrm{L}^{1}$ & 0.243 \\
& 428.46 & 0.0524 & 2.890 & $350-357$ & $\mathrm{H}^{-2}-\mathrm{L}^{4}$ & 0.340 \\
& 488.42 & 0.1281 & 2.538 & $351-354$ & $\mathrm{H}^{1}-\mathrm{L}^{1}$ & 0.447 \\
\hline $\mathbf{3}$ & 327.45 & 0.0268 & 3.786 & $332-350$ & $\mathrm{H}^{16}-\mathrm{L}^{3}$ & 0.366 \\
& 428.39 & 0.0165 & 2.894 & $342-351$ & $\mathrm{H}^{6}-\mathrm{L}^{2}$ & 0.187 \\
& 483.97 & 0.0672 & 2.561 & $346-349$ & $\mathrm{H}^{2}-\mathrm{L}^{4}$ & 0.529 \\
\hline $\mathbf{4}$ & 264.96 & 0.0123 & 4.679 & $103-109$ & $\mathrm{H}-\mathrm{L}^{5}$ & 0.562 \\
& 328.56 & 0.0350 & 3.773 & $96-105$ & $\mathrm{H}^{-9}-\mathrm{L}^{2}$ & 0.550 \\
& 432.97 & 0.0358 & 2.863 & $102-104$ & $\mathrm{H}^{-1}-\mathrm{L}$ & 0.671 \\
& 498.16 & 0.815 & 2.488 & $103-104$ & $\mathrm{H}^{-} \mathrm{L}$ & 0.689 \\
\hline
\end{tabular}


Table S9. Optimization of reaction conditions

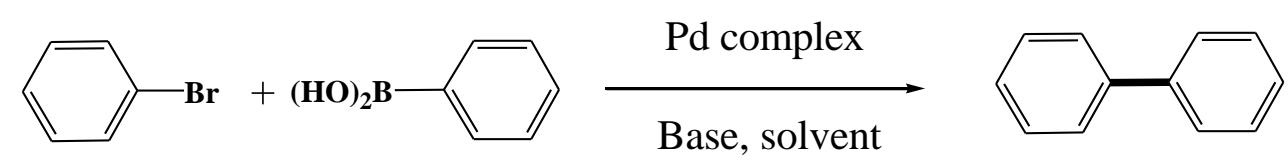

\begin{tabular}{|c|c|c|c|c|c|c|}
\hline Entry & Solvent (ratio) & $\begin{array}{l}\text { Base } \\
(\mathrm{mmol})\end{array}$ & $\begin{array}{l}\text { Catalyst } \\
\text { (mmol) }\end{array}$ & $\begin{array}{l}\text { Temperature } \\
\left({ }^{\circ} \mathrm{C}\right)\end{array}$ & $\begin{array}{l}\text { Time } \\
\text { (h) }\end{array}$ & $\begin{array}{l}\text { Yield } \\
(\%)^{\mathrm{a}}\end{array}$ \\
\hline 1 & $\mathrm{EtOH}-\mathrm{H}_{2} \mathrm{O}(4: 1)$ & $\mathrm{K}_{2} \mathrm{CO}_{3}(1.5)$ & $\mathbf{1}(0.005)$ & 70 & 6 & 76 \\
\hline 2 & $\mathrm{MeOH}-\mathrm{H}_{2} \mathrm{O}(4: 1)$ & $\mathrm{K}_{2} \mathrm{CO}_{3}(1.5)$ & $\mathbf{1}(0.005)$ & 70 & 6 & 72 \\
\hline 3 & DMF- $\mathrm{H}_{2} \mathrm{O}(4: 1)$ & $\mathrm{K}_{2} \mathrm{CO}_{3}(1.5)$ & $\mathbf{1}(0.005)$ & 70 & 6 & 65 \\
\hline 4 & DMAC- $\mathrm{H}_{2} \mathrm{O}(4: 1)$ & $\mathrm{K}_{2} \mathrm{CO}_{3}(1.5)$ & $\mathbf{1}(0.005)$ & 70 & 6 & 56 \\
\hline 5 & $\mathrm{CH}_{3} \mathrm{CN}-\mathrm{H}_{2} \mathrm{O}(4: 1)$ & $\mathrm{K}_{2} \mathrm{CO}_{3}(1.5)$ & $\mathbf{1}(0.005)$ & 70 & 6 & - \\
\hline 6 & $\mathrm{DMSO}-\mathrm{H}_{2} \mathrm{O}(4: 1)$ & $\mathrm{K}_{2} \mathrm{CO}_{3}(1.5)$ & $1(0.005)$ & 70 & 6 & 41 \\
\hline 7 & $\mathrm{CHCl}_{3}-\mathrm{H}_{2} \mathrm{O}(4: 1)$ & $\mathrm{K}_{2} \mathrm{CO}_{3}(1.5)$ & $\mathbf{1}(0.005)$ & 70 & 6 & - \\
\hline 8 & $\mathrm{EtOH}-\mathrm{H}_{2} \mathrm{O}(4: 1)$ & $\mathrm{KOH}(1.5)$ & $1(0.005)$ & 70 & 6 & 69 \\
\hline 9 & $\mathrm{EtOH}-\mathrm{H}_{2} \mathrm{O}(4: 1)$ & $\mathrm{NaOH}(1.5)$ & $\mathbf{1}(0.005)$ & 70 & 6 & 70 \\
\hline 10 & $\mathrm{EtOH}-\mathrm{H}_{2} \mathrm{O}(4: 1)$ & $\mathrm{Na}_{2} \mathrm{CO}_{3}(1.5)$ & $1(0.005)$ & 70 & 6 & 71 \\
\hline 11 & $\mathrm{EtOH}-\mathrm{H}_{2} \mathrm{O}(4: 1)$ & $\mathrm{Et}_{3} \mathrm{~N}(1.5)$ & $1(0.005)$ & 70 & 6 & 62 \\
\hline 12 & $\mathrm{EtOH}-\mathrm{H}_{2} \mathrm{O}(4: 1)$ & $\mathrm{K}_{2} \mathrm{CO}_{3}(1.5)$ & $\mathbf{1}(0.005)$ & 35 & 24 & Trace \\
\hline 13 & $\mathrm{EtOH}-\mathrm{H}_{2} \mathrm{O}(4: 1)$ & $\mathrm{K}_{2} \mathrm{CO}_{3}(1.5)$ & $\mathbf{1}(0.005)$ & 70 & 3 & 49 \\
\hline 14 & $\mathrm{EtOH}-\mathrm{H}_{2} \mathrm{O}(4: 1)$ & No base & $\mathbf{1}(0.005)$ & 70 & 6 & 25 \\
\hline 15 & $\mathrm{EtOH}-\mathrm{H}_{2} \mathrm{O}(4: 1)$ & $\mathrm{K}_{2} \mathrm{CO}_{3}(1.5)$ & No catalyst & 70 & 8 & Trace \\
\hline 16 & $\mathrm{EtOH}$ & $\mathrm{K}_{2} \mathrm{CO}_{3}(1.5)$ & $1(0.005)$ & 70 & 6 & 55 \\
\hline 17 & $\mathrm{H}_{2} \mathrm{O}$ & $\mathrm{K}_{2} \mathrm{CO}_{3}(1.5)$ & $1(0.005)$ & 70 & 6 & $<10$ \\
\hline 18 & EtOH-H2O (4:1) & $\mathrm{K}_{2} \mathrm{CO}_{3}(\mathbf{1 . 5})$ & $2(0.005)$ & 70 & 6 & 87 \\
\hline 19 & $\mathrm{EtOH}-\mathrm{H}_{2} \mathrm{O}(4: 1)$ & $\mathrm{K}_{2} \mathrm{CO}_{3}(1.5)$ & $3(0.005)$ & 70 & 6 & 49 \\
\hline 20 & $\mathrm{EtOH}-\mathrm{H}_{2} \mathrm{O}(4: 1)$ & $\mathrm{K}_{2} \mathrm{CO}_{3}(1.5)$ & $4(0.02)$ & 70 & 6 & 79 \\
\hline
\end{tabular}

Reaction conditions: aryl halide ( $1 \mathrm{mmol})$, phenyl boronic acid $(1.2 \mathrm{mmol})$, base $(1.5 \mathrm{mmol})$. ${ }^{\text {aIsolated yield. }}$ 
Table S10. Effect of solvent ratio

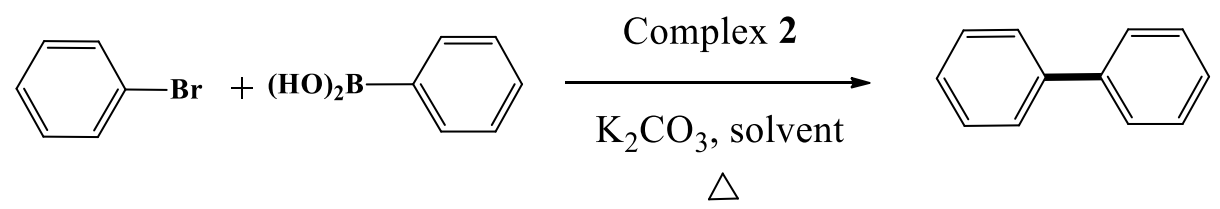

\begin{tabular}{llllll}
\hline Entry & Solvent (ratio) & $\begin{array}{l}\text { Catalyst } \\
(\mathbf{m m o l})\end{array}$ & $\begin{array}{l}\text { Temperature } \\
\left({ }^{\mathbf{0}} \mathbf{C}\right)\end{array}$ & $\begin{array}{l}\text { Time } \\
(\mathbf{h})\end{array}$ & $\begin{array}{l}\text { Yield } \\
(\boldsymbol{\%})^{\mathbf{a}}\end{array}$ \\
\hline 1 & $\mathrm{EtOH}-\mathrm{H}_{2} \mathrm{O}(4: 1)$ & $\mathbf{2}(0.005)$ & 70 & 3 & 87 \\
2 & $\mathrm{EtOH}-\mathrm{H}_{2} \mathrm{O}(3: 1)$ & $\mathbf{2}(0.005)$ & 70 & 3 & 77 \\
$\mathbf{3}$ & $\mathbf{E t O H}-\mathrm{H}_{2} \mathrm{O}(\mathbf{2 : 1 )}$ & $\mathbf{2 ( \mathbf { 0 . 0 0 5 } )}$ & $\mathbf{7 0}$ & $\mathbf{3}$ & $\mathbf{9 6}$ \\
4 & $\mathrm{EtOH}-\mathrm{H}_{2} \mathrm{O}(1: 1)$ & $\mathbf{2}(0.005)$ & 70 & 3 & 16 \\
5 & $\mathrm{EtOH}$ & $\mathbf{2}(0.005)$ & 70 & 5 & 60 \\
6 & $\mathrm{H}_{2} \mathrm{O}$ & $\mathbf{2}(0.005)$ & 70 & 5 & 10
\end{tabular}

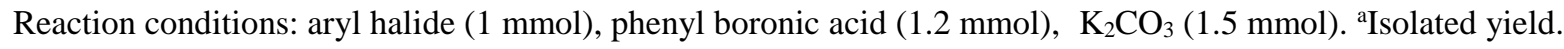

Table S11. Variation of catalyst loading

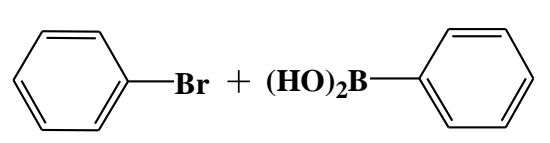

$$
\underset{\mathrm{K}_{2} \mathrm{CO}_{3}, \mathrm{EtOH} / \mathrm{H}_{2} \mathrm{O}(2: 1)}{\stackrel{\text { Complex } 2}{\longrightarrow}}
$$

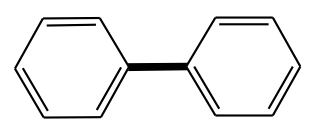

$\triangle$

\begin{tabular}{|c|c|c|c|c|c|}
\hline Entry & Solvent (ratio) & $\begin{array}{c}\text { Catalyst } \\
\text { (mmol) }\end{array}$ & $\begin{array}{c}\text { Temperature } \\
\left({ }^{\circ} \mathrm{C}\right)\end{array}$ & $\begin{array}{c}\text { Time } \\
\text { (h) }\end{array}$ & $\begin{array}{l}\text { Yield } \\
(\%)^{\mathrm{a}}\end{array}$ \\
\hline 1 & EtOH- $\mathrm{H}_{2} \mathrm{O}(2: 1)$ & $2(0.005)$ & 70 & 3 & 96 \\
\hline 2 & $\mathrm{EtOH}-\mathrm{H}_{2} \mathrm{O}(2: 1)$ & $2(0.0025)$ & 70 & 3 & 97 \\
\hline 3 & EtOH-H & $2(0.00125)$ & 70 & 3 & 97 \\
\hline
\end{tabular}

Reaction conditions: aryl halide $(1 \mathrm{mmol})$, phenyl boronic acid $(1.2 \mathrm{mmol}), \mathrm{K}_{2} \mathrm{CO}_{3}(1.5 \mathrm{mmol})$, EtOH-Water (2:1). asolated yield. 


\section{References}

S1 Castineiras, A.; Fernandez-Hermida, N.; Garcia-Santos, I.; Rodriguez, G. Neutral Ni ${ }^{\mathrm{II}}$, $\mathrm{Pd}^{\mathrm{II}}$ and $\mathrm{Pt}^{\mathrm{II}} \mathrm{ONS}$-pincer complexes of 5-acetylbarbituric-4N-dimethylthiosemicarbazone: synthesis, characterization and properties. Dalton Trans. 2012, 41, 13486-13495.

S2 Kalaiarasi, G.; Ruchi Jain, Puschman, H.; Poorna Chandrika, S.; Preethi, K.; Prabhakaran, R. New binuclear Ni(II) metallates containing ONS chelators: synthesis, characterisation, DNA binding, DNA cleavage, protein binding, antioxidant activity, antimicrobial and in vitro cytotoxicity. New. J. Chem. 2017, 41, 2543-2560.

S3 Kalaivani, P.; Prabhakaran, R.; Kaveri, M. V.; Huang, R.; Staples, R. J.; Natarajan, K. Synthesis, spectral, X-ray crystallography, electrochemistry, DNA/protein binding and radical scavenging activity of new palladium(II) complexes containing triphenylarsine. Inorg. Chim. Acta 2013, 405, 415-426.

S4 Boghaei, D. M.; Mohebi, S. Novel unsymmetrical tetradentate Schiff base complexes of cobalt (II) and palladium (II) with $\mathrm{N}_{2} \mathrm{O}_{2}$ donor sets J. Chem. Res. 2001, 6, 224-226.

S5 Kalaiarasi, G.; Rex Jeya Rajkumar, S.; Dharani, S.; Frank R. Fronczek, Muthukumar Nadar, M. S. A.; Prabhakaran, R. Cyclometallated ruthenium(II) complexes with 3-acetyl-2[H]-chromene-2-one derived CNS chelating ligand systems: synthesis, X-ray characterization and biological evaluation. New J. Chem. 2018, 42, 336-354.

S6 Kalaiarasi, G.; Rex Jeya Rajkumar, S.; Dharani, S.; Malecki, J. G.; Prabhakaran, R. An investigation on 3-acetyl-7-methoxy-coumarin Schiff bases and their $\mathrm{Ru}(\mathrm{II})$ metallates with potent antiproliferative activity and enhanced LDH and NO release. $R S C A d v$. 2018, 8, 15391561.

S7 Kalaivani, P.; Prabhakaran, R.; Poornima, P.; Dallemer, F.; Vijayalakshmi, K.; Vijaya Padma, V.; Natarajan, K. Versatile Coordination Behavior of Salicylaldehyde thiosemicarbazone in Ruthenium(II) Carbonyl Complexes: Synthesis, Spectral, X-ray, Electrochemistry, DNA Binding, Cytotoxicity, and Cellular Uptake Studies. Organometallics 2012, 31, 8323-8332.

S8 Prabhu, R. N.; Pandiarajan, D.; Ramesh, R. Ruthenium(II) mediated C-H activation of substituted acetophenone thiosemicarbazones: Synthesis, structural characterization, luminescence and electrochemical properties. J. Organomet. Chem. 2009, 694, 4170-4177. 
S9 Patil, S. A.; Unki, S. N.; Kulkarni, A. D.; Badami, V. H. N. Co(II), Ni(II) and Cu(II) complexes with coumarin-8-yl Schiff-bases: spectroscopic, in vitro antimicrobial, DNA cleavage and fluorescence studies. Spectrochim. Acta Part A 2011, 79, 1128-1136.

S10 Ali, A. A.; Nimir, H.; Aktas, C.; Huch, V.; Rauch, U.; Schafer, K. H.; Veith, M. Organoplatinum(II) Complexes with 2-Acetylthiophene Thiosemicarbazone: Synthesis, Characterization, Crystal Structures, and in Vitro Antitumor Activity. Organometallics 2256, 31, 2256-2262.

S11 Kalaivani, P.; Prabhakaran, R.; Ramachandran, E.; Dallemer, F.; Paramaguru, G.; Renganathan, R.; Poornima, P.; Vijaya Padma, V.; Natarajan, K. Influence of terminal substitution on structural, DNA, Protein binding, anticancer and antibacterial activities of palladium(II) complexes containing 3-methoxy salicylaldehyde-4(N) substituted thiosemicarbazones. Dalton Trans. 2012, 41, 2486-2499. 